\title{
British Association for Psychopharmacology consensus guidance on the use of psychotropic medication preconception, in pregnancy and postpartum 2017
}

\author{
R.H. McAllister-Williams ${ }^{1,2, *}$, D.S. Baldwin ${ }^{3,4}$, R. Cantwell ${ }^{5}$, A. Easter ${ }^{6}$, E.

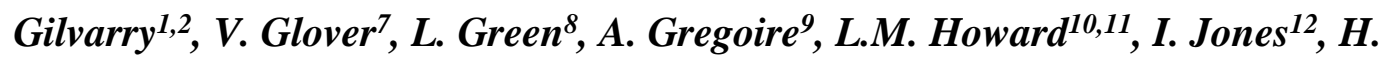 \\ Khalifeh $^{10,11}$, A. Lingford-Hughes ${ }^{13}$, E. McDonald ${ }^{14,15,16}$, N. Micali ${ }^{17}$, C.M.

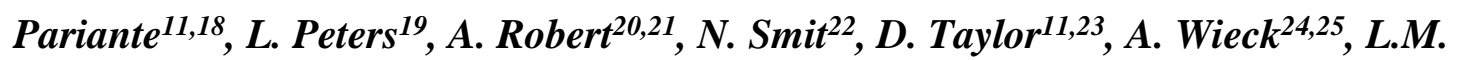 \\ Yates $^{26,27}$, A.H. Young ${ }^{11,18}$; endorsed by the British Association for \\ Psychopharmacology \\ * - Correspondence: Dr R.H. McAllister-Williams \\ Academic Psychiatry \\ Wolfson Research Centre \\ Campus for Ageing and Vitality \\ Newcastle upon Tyne \\ NE4 5PL \\ Tel: $\quad+44(0) 1912081370$ \\ Fax: $\quad$ +44 (0) 1912081387 \\ E-mail: $\quad$ r.h.mcallister-williams@ncl.ac.uk
}

\section{Author affiliations:}

1 - Institute of Neuroscience, Newcastle University, Newcastle, UK

2 - Northumberland Tyne and Wear NHS Foundation Trust, Newcastle upon Tyne, UK.

3 - Clinical and Experimental Sciences, Faculty of Medicine, University of Southampton, UK.

4 - University Department of Psychiatry and Mental Health, University of Cape Town, South Africa.

5 - NHS Greater Glasgow and Clyde, Glasgow, UK

6 - Centre for Implementation Science, Health Service and Population Research Department, Institute of Psychiatry, Psychology and Neuroscience, King's College London, UK 
7 - Institute of Reproductive and Developmental Biology, Imperial College London, UK

8 - Ealing, Hounslow,Hammersmith \& Fulham Perinatal Mental Health Service, West London Mental Health Trust, London, UK

9 - Hampshire Perinatal Mental Health Service, Winchester, UK

10 - Section of Women's Mental Health, Institute of Psychiatry Psychology and

Neuroscience, King's College London, London, UK

11 - South London and Maudsley NHS Foundation Trust, London, UK

12 - National Centre for Mental Health, MRC Centre for Neuropsychiatric Genetics and Genomics, Cardiff University, Cardiff, UK

13 - Centre for Psychiatry, Imperial College, London, UK

14 - Royal College of Psychiatrists, London, UK

15 - East London Foundation Trust, London, UK

16 - Tavistock and Portman NHS Foundation Trust, London, UK

17 - Behavioural and Brain Sciences Unit, GOSH Institute of Child Health,

University College London, London, UK

18 - Department of Psychological Medicine, Institute of Psychiatry, Psychology and Neuroscience, King's College London, London, UK

19 - St Martin's Healthcare Services CIC, Leeds, UK

20 - Hertfordshire Partnership University NHS Foundation Trust, Hatfield,

Hertfordshire, UK

21 - Postgraduate School of Medicine, University of Hertfordshire, Hatfield, Hertfordshire, UK

22 - Tees, Esk and Wear Valleys NHS Foundation Trust, Darlington, County

Durham, UK

23 - Institute of Pharmaceutical Science, King's College London, London, UK

24 - Greater Manchester Mental Health NHS Foundation Trust, Manchester, UK

25 - University of Manchester, Manchester, UK

26 - UK Teratology Information Service (UKTIS), Newcastle upon Tyne Hospitals

NHS Foundation Trust, Newcastle upon Tyne, UK

27 - Institute of Genetic Medicine, Newcastle University, Newcastle, UK 


\begin{abstract}
Decisions about the use of psychotropic medication in pregnancy are an ongoing challenge for clinicians and women with mental health problems, due to the uncertainties around risks of the illness itself to mother and fetus/infant, effectiveness of medications in pregnancy and risks to the fetus/infant from in utero exposure or via breast milk. These consensus guidelines aim to provide pragmatic advice regarding these issues. They are divided into sections on risks of untreated illness in pregnancy; general principles of using drugs in the perinatal period; benefits and harms associated with individual drugs; and recommendations for the management of specific disorders.
\end{abstract}

Key words: Psychotropics, antidepressants, antipsychotics, anxiolytics, mood stabilisers, hypnotics, fertility, conception, pregnancy, post-partum, breastfeeding, pregnancy outcome, neonatal problems, teratogenicity, child development, birth defects, psychiatric illness 


\section{TABLE OF CONTENTS}

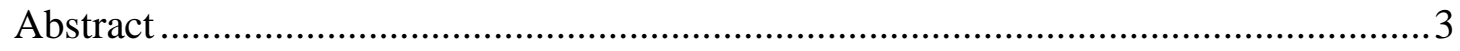

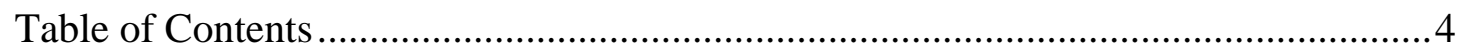

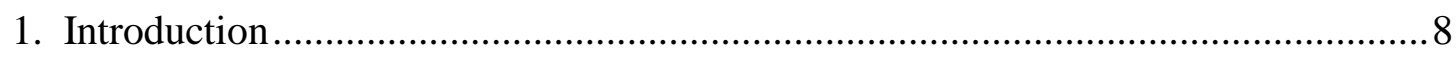

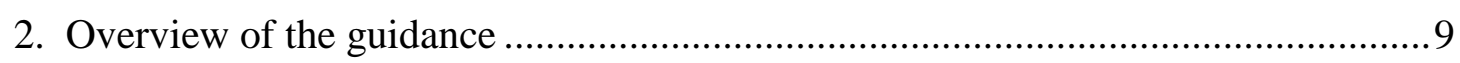

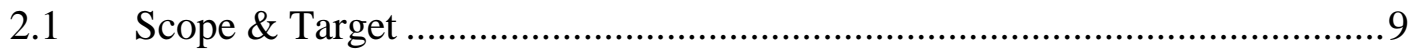

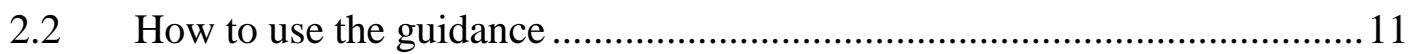

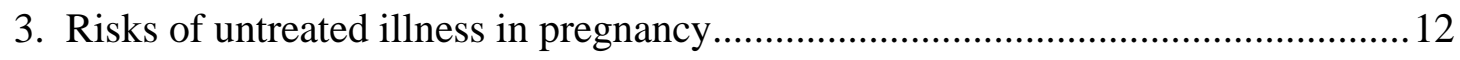

3.1 Risk of relapse during pregnancy and postnatal episodes of illness ............12

3.2 Impact of untreated mental illness on antenatal care and the woman's

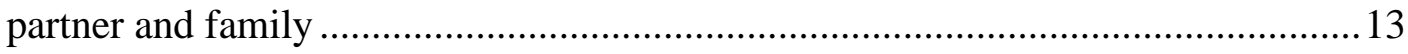

3.3 Risks related to untreated depression on pregnancy outcome and offspring

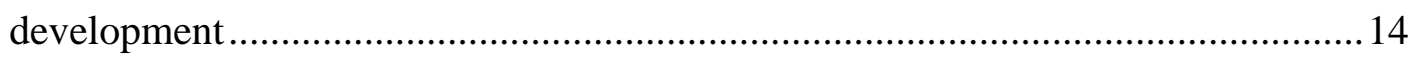

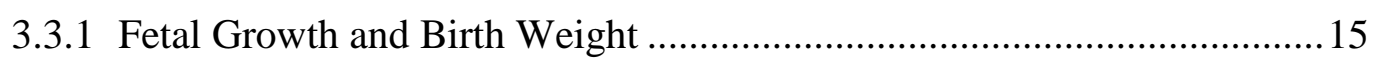

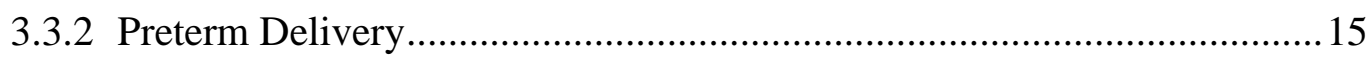

3.3.3 Other pregnancy and neonatal outcomes ................................................ 16

3.3.4 Risks of untreated antenatal illness on the infants development and future

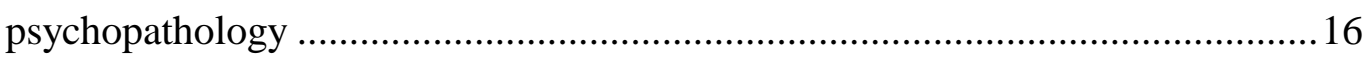

3.3.5 Impact of depression postnatally ...................................................... 18

3.4 Risks related to anxiety, stress and insomnia............................................19

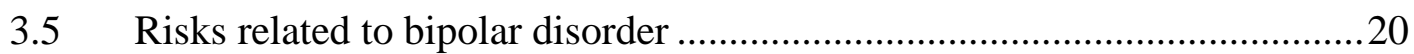

3.6 Risks related to schizophrenia and other psychotic illnesses.....................21

3.7 Risks of attention deficit hyperactivity disorder (ADHD) .........................22

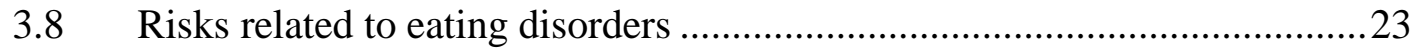

3.9 Risks related to substance misuse _.........................................................24

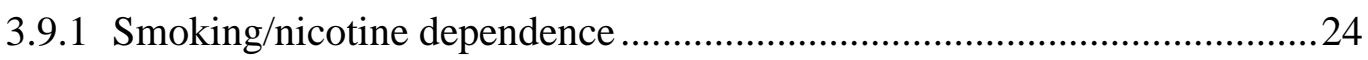

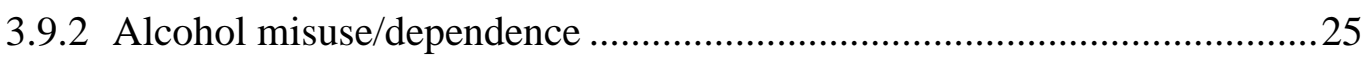

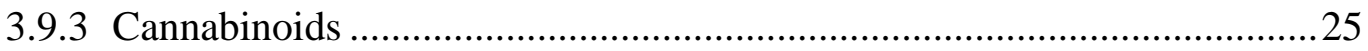

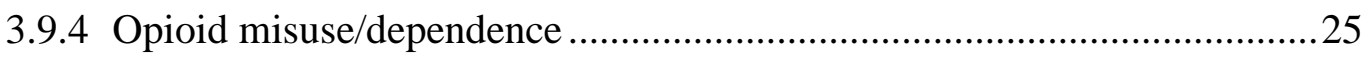

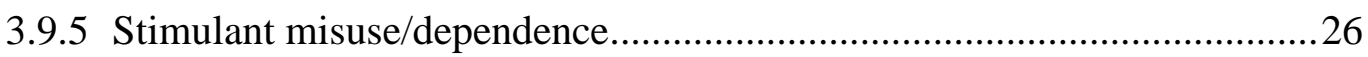

3.9.6 Benzodiazepine misuse/dependence...................................................26

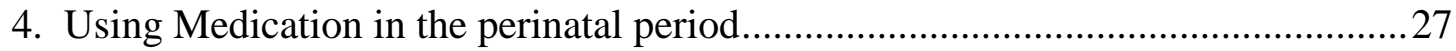

4.1 Weaknesse in the data on which perinatal guidelines are based ..................27 
4.2 Recommended principles of prescribing to women in the perinatal period.28

4.2.1 General management issues across all childbearing stages ......................28

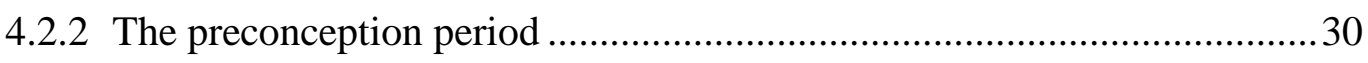

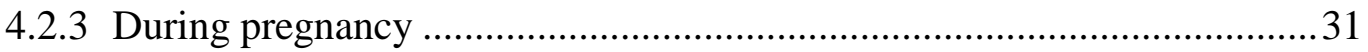

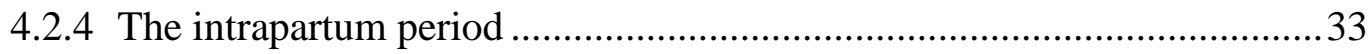

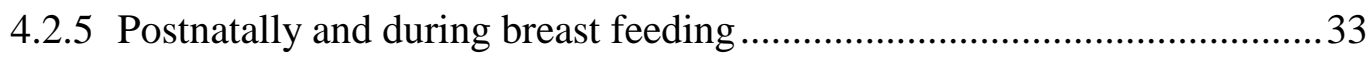

4.3 Recommendations regarding discussion of the risks and benefits of

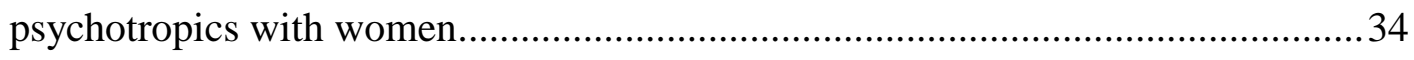

5. Benefits and harms associated with individual medications..................................37

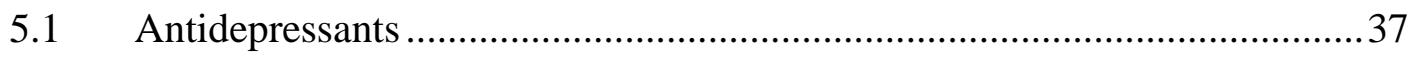

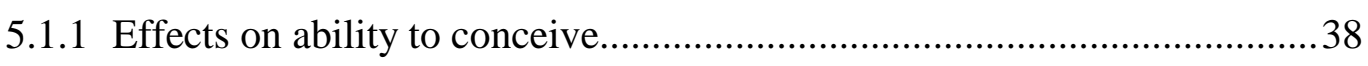

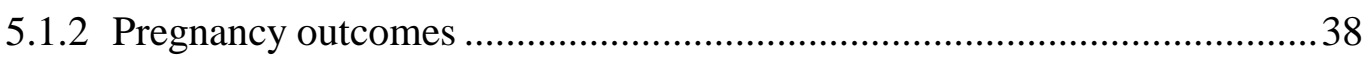

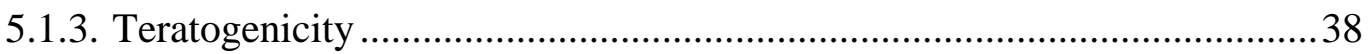

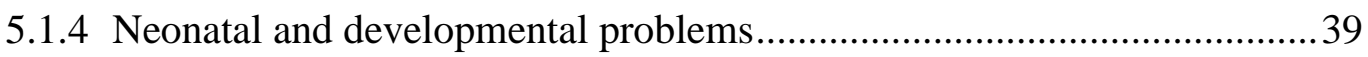

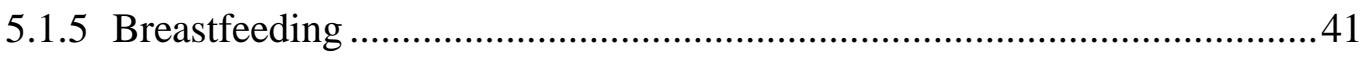

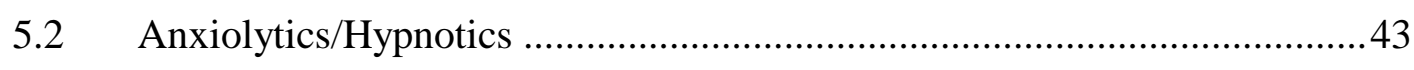

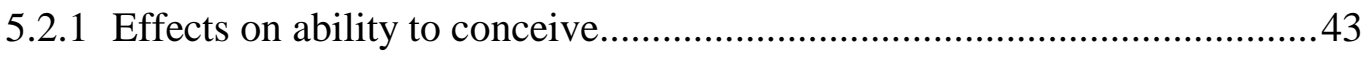

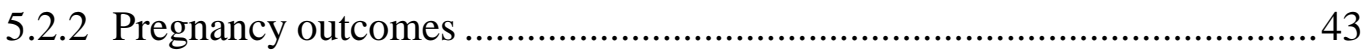

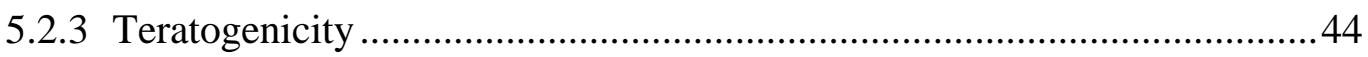

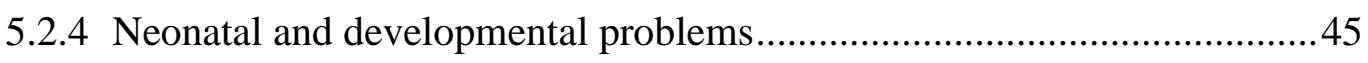

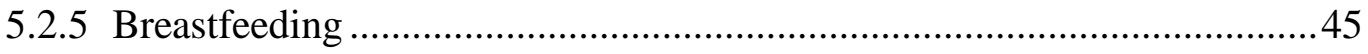

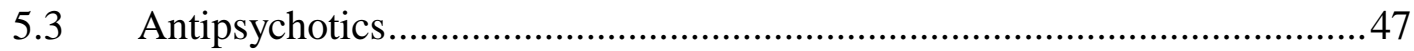

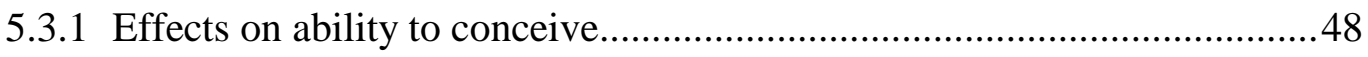

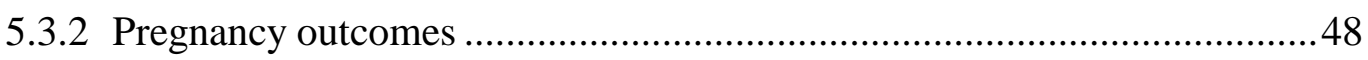

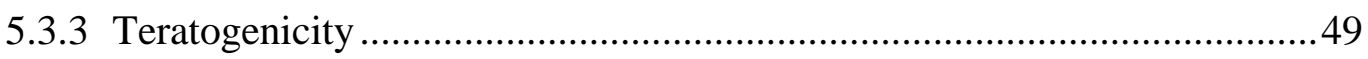

5.3.4 Neonatal and developmental problems..............................................50

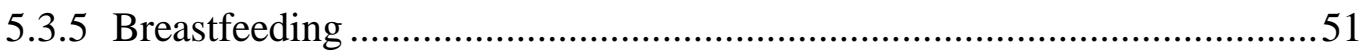

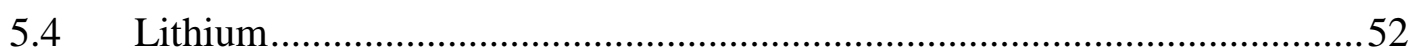

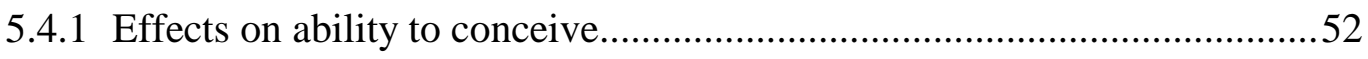

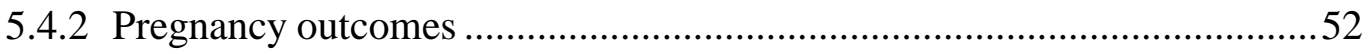

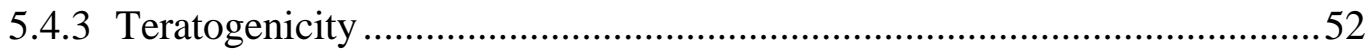

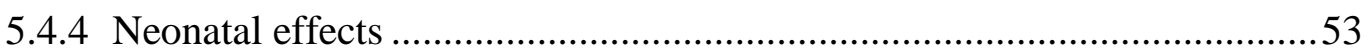

5.4.5 Neurodevelopmental effects ................................................................53 


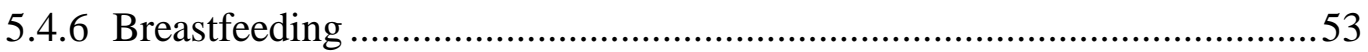

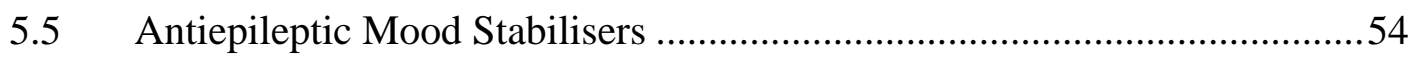

5.5.1 Endocrine and Effects on ability to conceive ........................................54

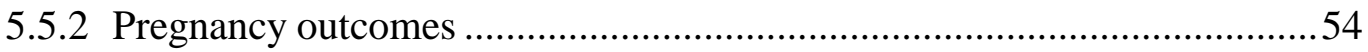

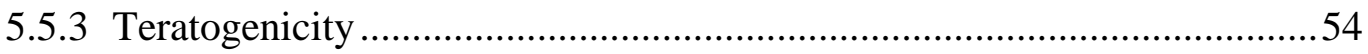

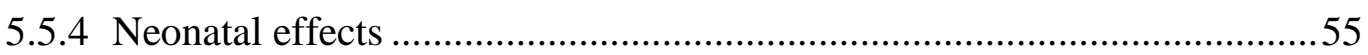

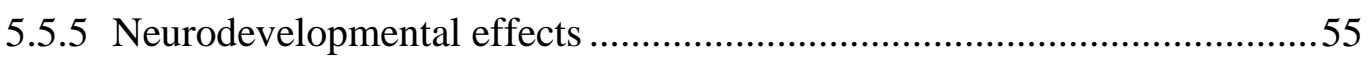

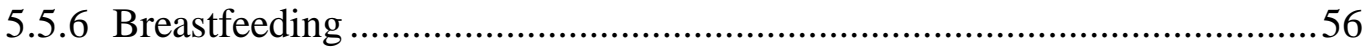

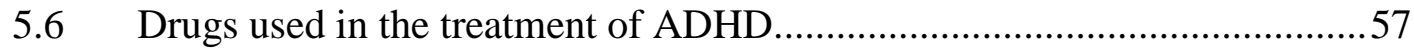

5.7 Drugs used in treatment of substance disorders........................................59

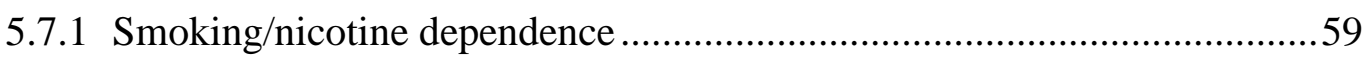

5.7.2 Alcohol misuse/dependence ...........................................................6

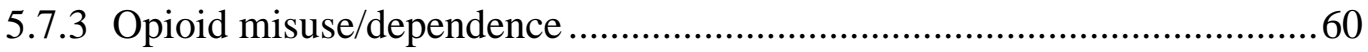

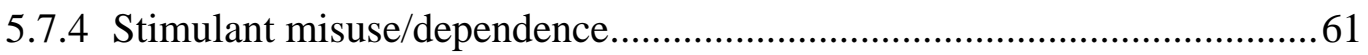

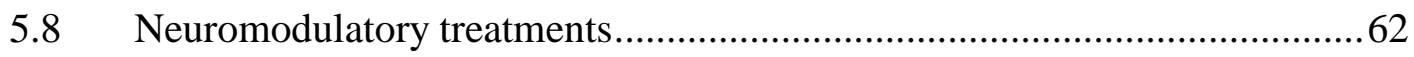

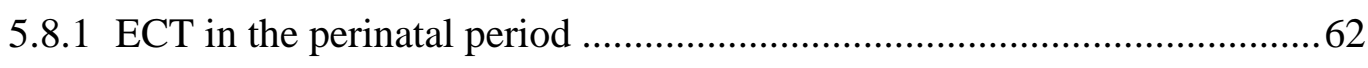

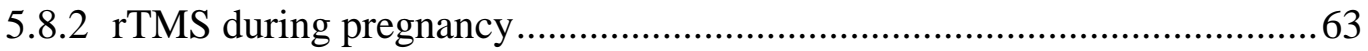

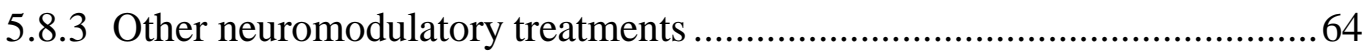

6. Recommendations for the pharmacological management of Specific disorders ....65

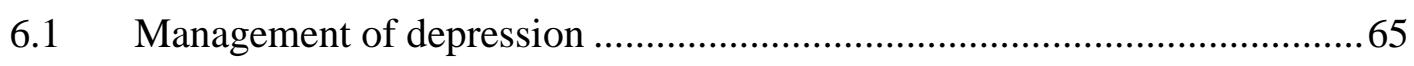

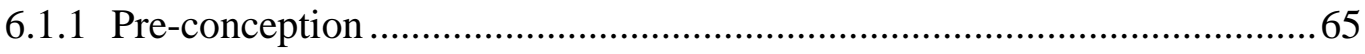

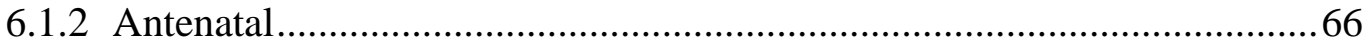

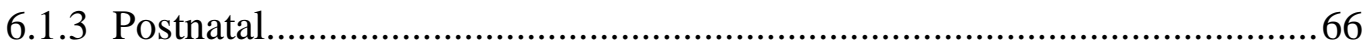

6.2 Management of anxiety disorders and insomnia..................................67

6.3 Management of bipolar disorder ...........................................................6 68

6.3.1 General management issues across all childbearing stages .....................68

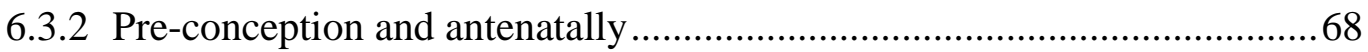

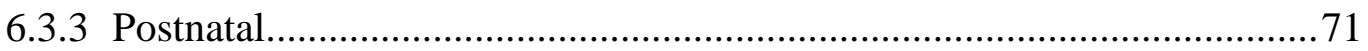

6.4 Management of schizophrenia and psychosis .......................................... 72

6.4.1 Management across all childbearing stages .......................................... 72

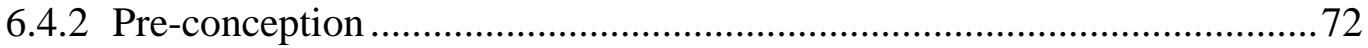

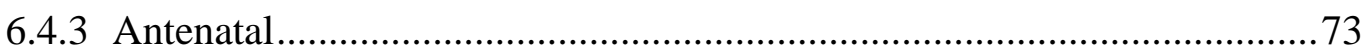

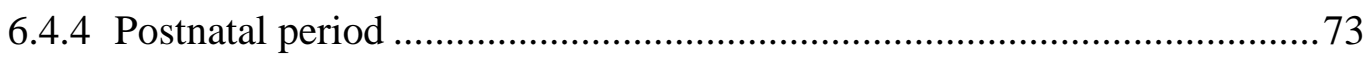




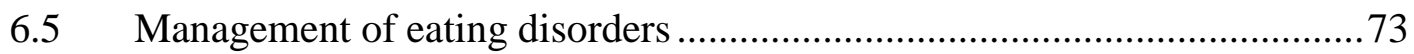

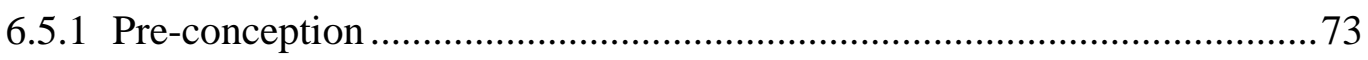

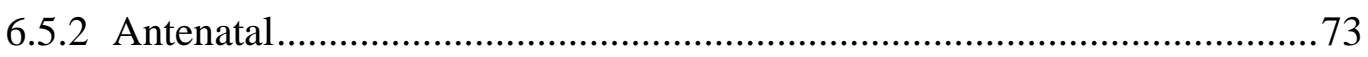

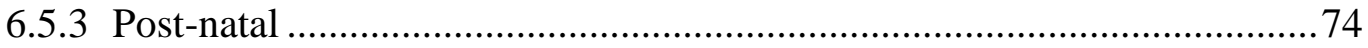

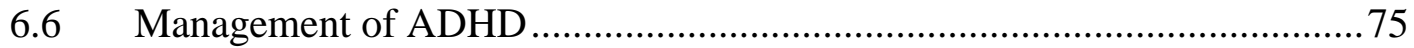

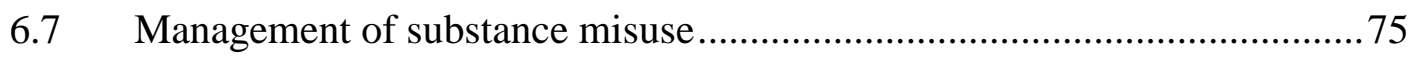

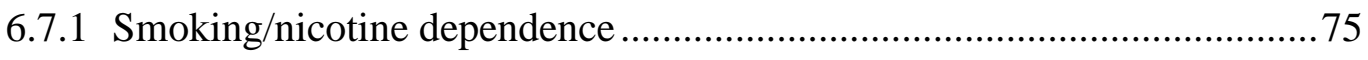

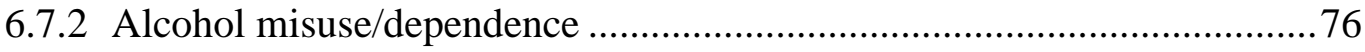

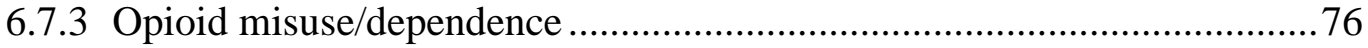

6.7.4 Stimulant misuse/dependence.............................................................. 77

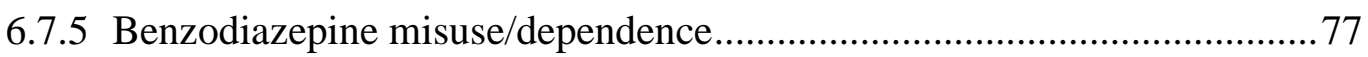

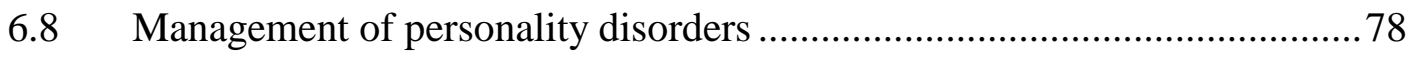

6.9 Management of acute behavioural disturbance..........................................78

7. Acknowledgements, funding and Declaration of Interest ...................................81

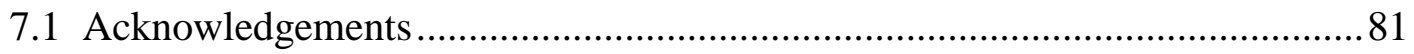

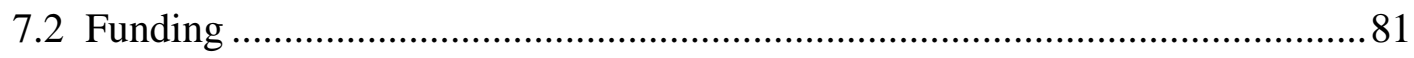

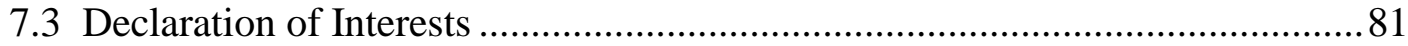

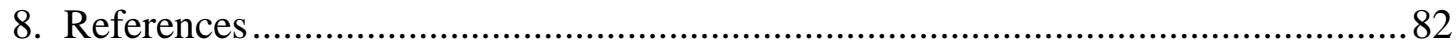




\section{INTRODUCTION}

The British Association for Psychopharmacology (BAP) has published a series of evidence-based guidelines for the use of drugs in patients with psychiatric disorders with the emphasis on producing comprehensive, concise and useable guidance based on a review of the relevant evidence (see www.bap.org.uk). This new guideline relates to the use of psychotropic medication during the perinatal period (see definition of this below).

Guidelines proliferate in areas of clinical uncertainty. While there have been many studies published in recent years, there remains a paucity of high quality or consistent evidence to guide psychotropic prescribing in the perinatal period. So clinicians are perhaps more dependent on guidelines in this area than in others. In the UK, three main bodies produce sets of guidelines exist regarding the use of psychotropics: the National Institute for Health and Care Excellence (NICE), the Scottish Intercollegiate Guideline Network (SIGN) and the BAP. Advice relating to prescribing in pregnancy is also available from the UK Teratology Information Service (UKTIS).

The NICE perinatal guidelines repeatedly emphasise the need for access to advice from experts regarding the use of psychotropics in the perinatal period (NICE CG192, statement 1.10.3). This is not always readily available. The goal of these BAP guidelines is to provide concise and specific guidance for health care professionals.

\subsection{Methodology employed in creating this guidance}

A consensus meeting was held under the auspices of the BAP in 2012 involving experts in the field of perinatal psychiatry, psychopharmacology, teratology, infant and child development and service user representatives. Current clinical practice, existing UK guidance and key studies relevant to undertaking a risk/benefit analysis of psychotropic use in the perinatal period were critically reviewed. Given the high levels of anxiety around the use of medication in the perinatal period, the concensus group were keen to achieve a guideline to support evidence based shared decision making between clinican and patient. Development of this BAP guideline was paused pending the publication of NICE guidelines in 2015 (National Institute for Health and Care Excellence, 2015a) to ensure that patients and clinicians were not 
faced with inconsistent advice. The residual need for complimentary guidance that included practical clinical recommendations was however clear and work on the BAP guidelines was continued.

These BAP guidelines are based upon a selective review of key relevant studies, reviews and guidelines, including those from NICE, SIGN (Scottish Intercollegiate Guidelines Network, 2012), other guidelines published in 2014 and 2015 such as those from British Columbia in Canada (BC Reproductive Mental Health Programme and Perinatal Services BC, 2014) and from Denmark (Larsen et al., 2015), UKTIS published monographs (https://www.toxbase.org/), and consensus amongst the authors following iterative circulation of the guideline manuscript.

Given the broad scope of the literature on the risks of all classes of psychotropic drugs in the perinatal period, the risk/benefit ratios for pharmacological treatments across all psychiatric disorders (in pregnancy and, when this is not available, in non-pregnant women and men) and the impact of these disorders themselves on fetal and infant outcomes, it was not possible for the consensus group to undertake a comprehensive review of the entire evidence base. Rather there has been a focus on summarising data and making recommendations

\section{OVERVIEW OF THE GUIDANCE}

\section{$2.1 \quad$ Scope \& Target}

The term "perinatal" is used inconsistently in different situations. In these guidelines, for brevity, 'perinatal' is used to include the entire period of pregnancy, the preconception period and the postnatal period (conventionally consider the first year after birth).

These guidelines cover the main psychiatric disorders in which psychotropics are prescribed in adulthood. The focus is on the factors that guide treatment. Identification and assessment of psychiatric illnesses in the perinatal period are not covered. For these issues the reader is referred to the NICE Antenatal and Postnatal Mental Health guidelines (National Institute for Health and Care Excellence, 2015a). The focus is specifically on psychopharmacological treatments, but with reference to neuromodulatory treatments, particularly electroconvulsive therapy (ECT) and repetitive transcranial magnetic stimulation (rTMS). 
The intention of these guidelines is to support and facilitate evidence based practice. There are rarely unequivocally 'correct' or 'incorrect' decisions in the treatment of psychiatric disorders. Given the complexity of the risk/benefit equations when dealing with prescribing in pregnancy, this is even more the case. As a result these guidelines should not be read as a definitive set of instructions as to what to do or not do. The consensus group's strong view was that the generic factors that need to be considered in relation to prescribing in the perinatal period form the most important section in the guideline given the relatively few situations in which the evidence is such to allow for a categorical recommendation.

To compliment NICE (National Institute for Health and Care Excellence, 2015a) this review focuses on the evidence necessary to address NICE guidelines statement 1.4.3:

"Discuss treatment and prevention options and any particular concerns the woman has about the pregnancy or the fetus or baby. Provide information to the woman and, if she agrees, her partner, family or carer, about:

- the potential benefits of psychological interventions and psychotropic medication

- the possible consequences of no treatment

- the possible harms associated with treatment

- what might happen if treatment is changed or stopped, particularly if psychotropic medication is stopped abruptly."

There are few data regarding the benefits of psychotropics specifically in pregnancy due to the difficulties of conducting randomised controlled trials (RCTs) in this area. As a result it is usually necessary to extrapolate from population data regarding the efficacy of medications in specific disorders generally. The reader is directed to relevant BAP and NICE guidelines regarding these. Similarly, there are few data on the risks of stopping medication specifically in the perinatal period. Again there is a need to extrapolate data from the more general use of drugs. Consequently this guidance focuses particularly on the possible consequences of no treatment and the potential harms associated with treatment. 


\subsection{How to use the guidance}

The guidance comprises four main parts: Section 3 describes the risks to mothers and their children of psychiatric illness particularly when untreated. Section 4 relates to general principles of using medication in the perinatal period. Section 5 reviews the risks associated with specific psychotropic medications. Section 6 provides guidance on the management of psychiatric disorders in the perinatal period.

The guideline uses the convention of presenting summary conclusions and recommendations at the end of sub-sections in the form of bullet points in italics. Note that sections 4.2, 4.3 and 6 are entirely comprised of conclusions/recommendations. All recommendations have been refined iteratively through reviews of drafts of this paper.

Many readers will use this document to obtain guidance about the management of a specific condition. However, we would recommend that the reader first reviews the general principles described in Section 4. Subsequently, guidance in Section 6 should be supplemented with the relevant information described in Sections 3 on the possible consequences of no treatment and in Section 5 regarding the potential harms associated with treatment, together with guidance in other disorder specific BAP guidelines.

Unlike other BAP guidelines, this guidance does not describe the strength of evidence supporting the recommendations made. This is primarily because the conventional hierarchy of evidence is not applicable to much of the perinatal psychopharmacology field given the lack of RCTs. Most recommendations would be classed as 'standards of good practice' based on the collective view of the consensus group. Additionally, throughout the guideline statements regarding the magnitude of effects have been approximated to the nearest 'round' number to avoid the impression of precision, which is rarely the case in the perinatal literature, and/or due to inconsistencies between studies. A convention has been used where percentage increased or decreased risk is used for amounts of less than $100 \%$ and for amounts greater than this the change is reported as an $\mathrm{x}$-fold difference. In addition to the change in risk, which can be misleading when presented in isolation, absolute risks (e.g. with and without treatment) are presented to help interpretation.

Data informing these guidelines are constantly emerging. Prescribers are advised to seek advice as to whether there is information that would modify the 
recommendations made in these guidelines that has become available subsequent to their publication. Information on fetal risk may be obtained from UKTIS (http://www.uktis.org/). This guideline will be updated and re-published in due course. If the authors identify recommendations that need to be modified in the interim, these will be posted at http://www. bap.org.uk/perinatalupdates.

\section{RISKS OF UNTREATED ILLNESS IN PREGNANCY}

The importance of this issue is underscored by a recent UK analysis that has found that one in 25 women aged 20-35 who commit suicide do so in the perinatal period. Importantly, these women were twice as likely to be actively receiving mental health care (Kim and Silver, 2016).

Much of the data regarding the risks of untreated mental illness during pregnancy relates to mothers with anxiety and/or depression, with only limited information on other illnesses.

\subsection{Risk of relapse during pregnancy and postnatal episodes of illness}

Pregnancy is not protective against mental illness. If a mental illness is untreated because the woman makes a decision to discontinue psychotropic medication prior to, or soon after conception, there may be an increased risk of relapse. A variety of studies suggest this may depend on the severity of the illness as indicated, for example, by the level of services with which she is engaged. Very high rates of relapse of depression $(70 \%)$ have been found in some studies (Cohen et al., 2006; Cohen et al., 2004) when women have discontinued antidepressant medication. These studies were conducted in psychiatric settings and recruited women with histories of recurrent depression. However, in a study recruiting from obstetric settings, likely to include women with milder forms of depression, the risk for onset of a major depressive episode was similar in women whether or not they continued antidepressant medication (Yonkers et al., 2011).

Mental illness in pregnancy may persist postnatally. Depression in pregnancy is one of the strongest predictors of depression postnatally (Robertson et al., 2004). Additionally, antenatal poor mental health may increase the risk of different/additional postnatal mental illness. For example, up to one third of women with active bulimia 
in pregnancy also develop depression postnatally (Franko et al., 2001; Morgan et al., 2006).

There are few studies addressing the impact of pregnancy on severe mental illnesses (Jones et al., 2014). Although some studies have reported lower prevalence of new onset and recurrence of both schizophrenia and bipolar disorder in pregnancy (Munk-Olsen et al., 2009; Munk-Olsen et al., 2006) other studies, at least for bipolar disorder, have reported very high prevalence of recurrence (e.g. 85\%) in women discontinuing prophylactic medication (Viguera et al., 2007b).

\section{$\underline{\text { Conclusions/Recommendations }}$}

- Pregnancy is not protective against episodes of mental illness.

- Findings on the risk of relapse of unipolar depression if medication is stopped during or prior to pregnancy are inconsistent, possibly depending on severity of illness.

- Poor mental health in pregnancy, is a strong predictor of mental illness postnatally.

- It is uncertain whether treatment of antenatal mental illness decreases the risk of onset or exacerbation of illness postnatally but it is reasonable to assume this is the case.

\subsection{Impact of untreated mental illness on antenatal care and the woman's partner and family}

Mental illnesses are associated with a range of behaviours that have a negative impact on pregnancy outcome. Depressed women appear more likely to smoke, drink alcohol or use illicit substances in pregnancy (Shah and Howard, 2006; Zhu and Valbo, 2002; Zuckerman et al., 1989); the same observation applies to pregnant women with bipolar disorder, psychosis and eating disorders (Jones et al., 2014; Micali et al., 2016a).

Self- neglect and lack of motivation in depression, and other mental disorders, may also lead to poor self care and diet. Kim and colleagues (2006) found that 
women with a self-reported past history of psychiatric disorder delayed seeking antenatal care and were at higher risk of inadequate antenatal care.

A woman's partner and family are also likely to be affected if she is mentally unwell. For example, maternal depression is known to be a risk factor for paternal depression in the postnatal period (Bradley and Slade, 2011; Burke, 2003). Paternal mental illness may in turn affect the interaction between the father and the baby, and the implications for the baby and other children of both parents being unwell needs to be considered.

\section{$\underline{\text { Conclusions/Recommendations }}$}

- Women with depression, bipolar disorder, eating disorders or schizophrenia are more likely to misuse alcohol, nicotine and other substances during pregnancy which could negatively impact pregnancy outcomes.

- Women with mental illnesses may utilise antenatal care in sub-optimal ways.

- Maternal depression affects the whole family and increases the risk of paternal mental illness.

\subsection{Risks related to untreated depression on pregnancy outcome and offspring development}

Although there is evidence for an association between depression in pregnancy and an increased risk of adverse pregnancy and neonatal outcomes, including pre-term delivery and low infant birth weight (Alder et al., 2007; Grote et al., 2010), there are few studies which compare women with untreated depression to women taking antidepressant medication. This is the information women need in order to weigh the risks and benefits of treatment. Amongst these studies, there are a number of methodological issues which make comparison and meta-analysis challenging, including the fact that women who continue on antidepressants in pregnancy may represent a more severely unwell group but also may have lower depression scores and better functioning while on antidepressants. Additionally there is the issue of shared genetic risk, which may be particularly pertinent when considering offspring development. 


\subsubsection{Fetal Growth and Birth Weight}

The majority of studies comparing the offspring of mothers with untreated depression with those of non-depressed mothers report an association between maternal depression and restricted fetal growth and lower birth weight (Davalos et al., 2012; Diego et al., 2009; Field et al., 2006; Field et al., 2008; Steer et al., 1992). Diego et al (2009) found that $20 \%$ of infants born to mothers with antenatal depression had low birthweight compared to $5 \%$ in the non-depressed group. Steer et al (1992) found that risk of low birth weight rose 5-7\% for each additional Beck Depressive Inventory point when the mother rated the severity of her depression.

In studies which compare neonates of unmedicated depressed mothers to mothers treated with antidepressants the results are less conclusive (Davalos et al., 2012). Using population based linked health data, it has been reported that neonates exposed to SSRIs $(n=1451)$ and neonates exposed to untreated depression $(n=14234)$ both had lower birth weights than control neonates $(n=92192)$ (Oberlander et al., 2006). When outcomes were compared between SSRI exposed and non-exposed neonates (matched in terms of severity of depressive illness the mother suffered from), SSRI exposure in pregnancy was associated with an increased incidence of birthweight below the $10^{\text {th }}$ percentile. The authors suggested that SSRI exposure and depressed maternal mood had an additive negative effect (Oberlander et al., 2006). In contrast, two other studies, albeit with much smaller samples of women, have found no difference in birthweight between infants of mothers with untreated depression in pregnancy, those whose mothers were treated with SSRIs in pregnancy and a control group whose mothers were not depressed (Suri et al., 2004; Wisner et al., 2009).

\subsubsection{Preterm Delivery}

In studies comparing infants born to unmedicated depressed mothers with those of a non-depressed control group, the majority of studies report shorter length of gestation (Davalos et al., 2012). Preterm delivery (defined here and elsewhere in the guideline as delivery before 37 weeks gestation) is around three times as likely for depressed versus non-depressed mothers with absolute rates being approximately $25 \%$ for depressed and 7-10\% for non-depressed mothers (Diego et al., 2009; Field et al., 2006; Steer et al., 1992). The risk of pre-term delivery appears to be influenced by the severity of depression ( $\mathrm{Li}$ et al., 2009). A meta-analysis identified five studies of 
antenatal depression and risk of preterm birth for which stratification by antidepressant medication was possible and found comparable gestational ages for depressed women treated and not treated with antidepressants (Grote et al., 2010).

\subsubsection{Other pregnancy and neonatal outcomes}

Data linking untreated depression with risks of other outcomes are sparse and results inconsistent. Untreated depression in pregnancy has been associated with increased rates of Caesarean section in some studies (Chung et al., 2001; Oberlander et al., 2006), but not others (Wisner et al., 2009). Higher rates of neonatal intensive care unit admissions were found in some studies (Chung et al., 2001) but again not in others (Suri et al., 2004; Wisner et al., 2009).

\subsubsection{Risks of untreated antenatal illness on the infants development and future psychopathology}

Much of the data on this issue comes from women with depressive symptoms and anxiety not necessarily meeting criteria for a major depressive episode or an anxiety disorder. Additionally the shared genetics between the mother and the child is a confounder that is usually not addressed in the research described below.

Maternal antenatal depression and anxiety symptoms are reported to be associated with an increased risk of neurodevelopmental and psychopathological consequences for the offspring spanning into childhood and adolescence (Talge et al., 2007). This is thought to be, at least in part, due to fetal programming: the effect of the uterine environment, during specific critical periods for different behavioural outcomes, on fetal development with long term effects on the child (Sandman et al., 2011). Several studies have shown that these altered outcomes are independent of many possible confounding factors, including smoking, alcohol consumption and anxiety and depression postnatally, suggesting a possible causal associations with maternal illness during pregnancy (O'Donnell et al., 2014). Most children are not affected, and those that are can be affected in different ways, probably due to different genetic vulnerabilities and the quality of postnatal care. The neurodevelopmental outcomes for which there is evidence of an increased risk amongst offspring of women with anxiety or depressive symptoms during pregnancy include emotional problems, symptoms of ADHD and conduct disorder, impaired cognitive function, 
and schizophrenia (Glover, 2014). There are some reports of an increased risk of autism, but the evidence is less consistent (Glover, 2014).

In the large ALSPAC population study, it was found that if the mother was in the 'top' $15 \%$ for symptoms of anxiety or depression in pregnancy, her child was at double the population risk (of 5-10\%) for emotional and behavioural problems at ages 4 and 7, including symptoms of ADHD and conduct disorder (O'Connor et al., 2003). Similar results are seen when the child is 13 years old (O'Donnell et al., 2014).

In the only longitudinal study so far that has followed-up offspring of mothers who were diagnosed with depression during pregnancy up to adolescence, antenatal depression was associated with an increased risk of both depression and antisocial behaviour in the offspring (Pawlby et al., 2011; Pawlby et al., 2009), an effect that was specific to antenatal depression and was not present in the offspring of mothers who were only depressed postnatally (Hay et al., 2008). The risk of developing depression in the adolescent offspring of a mother who had suffered from antenatal depression was approximately 3 -fold that of control offspring (approximate absolute risk of $52 \%$ vs $24 \%$ ) though the sample size was small and hence the $95 \%$ confidence interval for the increased risk was wide (Pawlby et al., 2011; Pawlby et al., 2009). Data collected in the same offspring at age 25 were consistent with these earlier findings (Plant et al., 2015a). Evidence from the same study also shows that antenatal depression increases the risk of the offspring being exposed to maltreatment in childhood, by approximately 4-fold (Pawlby et al., 2011), especially if the mother was herself exposed to maltreatment in childhood (Plant et al., 2013). This increased risk was not due to the depressed mothers themselves being more likely to be perpetrators of the maltreatment, but rather due to an increased risk of maltreatment from other adults in the immediate environment.

The effect of the emotional state of the mother during pregnancy on fetal neurodevelopment is not confined to those with a diagnosed mental illness and other forms of stress also appear to have an effect. For example, in a study correlating antenatal life events with child cognitive development at 17 months, Bergman et al. found that antenatal stress accounted for $17 \%$ of the variance in cognitive ability after allowing for a range of confounders, including postnatal maternal mood (Bergman et al., 2007). 


\subsubsection{Impact of depression postnatally}

Maternal depression has been associated with various patterns of interactions with their babies that are, in turn, associated with disordered learning and development. These overlapping maternal behaviours can be characterised in three groups: withdrawn interactions, where the parent does not actively engage with the baby; hostile and intrusive interactions; and general sadness and insensitivity (Sutton et al., 2012). A lack of active, responsive, engagement with the infant is associated with poor infant learning and poorer cognitive development. These early effects tend to persist throughout childhood. Boys of mothers who were depressed do not achieve as well at age 16 in public exams as others (Murray et al., 2010).

\section{$\underline{\text { Conclusions/Recommendations }}$}

- There is limited evidence regarding the risks of untreated, as opposed to treated, depression on the pregnancy and infant outcomes.

- Untreated depression may be associated with low birth weight. The magnitude of this risk appears to correlate with severity of illness. It is however unclear if this risk is altered by treatment with antidepressants.

- Untreated depression may be associated with an increased risk of preterm delivery. Again the risk appears to correlate with severity of depression. Antidepressant treatment does not seem to influence the risk once women are matched on severity of depression.

- No firm conclusions can be drawn about potential associations of untreated depression with any other adverse pregnancy outcome.

- Antenatal maternal depression and anxiety has been associated with a range of adverse outcomes for the child. These include emotional problems, symptoms of ADHD, conduct disorder, impaired cognitive function, schizophrenia and possibly autism.

- Antenatal, but not postnatal, depression is associated with an increased risk of depression and antisocial behaviour in offspring. It is unknown if treatment of antenatal maternal ill health decreases these risks. 
- Depression postnatally can lead to poor engagement with infants, which in turn can lead to poor infant learning and cognitive development, with these effects persisting throughout childhood.

- There is a lack of evidence that treating depression postnatally can reduce these risks.

- The adverse effects of maternal antenatal and postnatal ill health appear to be additive.

\subsection{Risks related to anxiety, stress and insomnia.}

There is a great deal of overlap between depression and anxiety and much of the information in section 3.3 (especially section 3.3.4) potentially relates to both.

Anxiety disorders are common in the perinatal period, but often over-looked. The experience of childbirth can be so emotionally intense as to to precipitate the development of post-traumatic-like symptoms in approximately one-quarter of women after vaginal deliveries (Olde et al., 2006). Some observational studies suggest a higher incidence of obsessive-compulsive disorder (OCD) in the post-partum period, though data from prospective studies are limited, and possibly not generally applicable (McGuinness et al., 2011; Speisman et al., 2011).

Post-natal anxiety in mothers is possibly associated with childhood somatic (such as recurrent abdominal pain) and psychological (such as emotional and conduct difficulties) difficulties (Glasheen et al., 2010).

A systematic review suggests self-reported poor sleep during pregnancy (and in the postpartum year) is associated with an increased risk of post-partum depression: the association being less marked in studies in which sleep was assessed objectively (Lawson et al., 2015).

A systematic review and meta-analysis which found no evidence for the potential benefit of 'preventive' antenatal interventions (including educational classes and group antenatal care) in non-distressed women, did find evidence for the potential benefit of treatment interventions (including relaxation and mindfulness) in women who were already distressed (Fontein-Kuipers et al., 2014). There is also evidence that psychological interventions designed to enhance coping and self-regulation in 
women following pre-term birth reduces maternal depressive and anxiety symptoms and parenting stress (Kraljevic and Warnock, 2013). However routine screening for the presence of anxiety is challenging, with no scale having sufficiently robust psychometric properties for routine clinical use (Brunton et al., 2015); similar problems are seen when assessing 'psychosocial stress' during pregnancy (Nast et al., 2013).

Conclusions/Recommendations

- Anxiety and stress in mothers during pregnancy can be associated with long term behavioural and mental health problems in offspring.

- There is less evidence regarding insomnia but it may be associated with an increased risk of postpartum depression.

- It is unclear whether antenatal interventions to treat anxiety and insomnia impact on infant outcomes.

- Anxiety disorders can be problematic postnatally. There is doubt as to whether the incidence of OCD is increased or not. PTSD however can present, especially after traumatic deliveries.

\subsection{Risks related to bipolar disorder}

Pregnancy does not protect from recurrences of bipolar disorder. In a prospective cohort study of pregnant women with bipolar disorder who were well, taking prophylactic mood stabilizers, those who discontinued the medication proximate to conception had a two-fold higher recurrence risk ( $86 \%$ vs 37\%), a fourfold shorter time to first recurrence ( 9 vs more than 40 weeks) and a 4 to 5 -fold increased time spent ill in pregnancy than those who remained on the medication (40\% vs $9 \%$ of the pregnancy) (Viguera et al., 2007b).

Immediately after childbirth the risk of postpartum psychosis (including mania, mixed affective states and psychotic depression) and episodes of bipolar disorder increases in women with bipolar disorder, but there is some debate as to what extent. A recent meta-analysis of studies reporting on postpartum recurrences found that the rates varied widely; for bipolar disorder between 9 and 75\%; and for puerperal psychosis between 14 and 57\% (Wesseloo et al., 2016). This may well reflect 
differences in definitions of recurrence, the range of phenotypes being included and, importantly, whether women were or were not on medication. Women with bipolar disorder who were medication free during pregnancy had a significantly higher risk of postpartum relapse (approximately 65\% versus 25\%) than those who were taking prophylactic medication (Wesseloo et al., 2016).

A population based cohort study in Sweden provides the only evidence on outcomes for infants of mothers with untreated bipolar disorder in pregnancy. The data are confounded because mothers with bipolar disorder were more often smokers, overweight and alcohol or substance abusers, regardless of treatment, when compared to unaffected mothers (Boden et al., 2012a). Both untreated and treated women with bipolar disorder had increased risks of Caesarean delivery, instrumental delivery and a non-spontaneous start to delivery, as well as pre-term delivery ( $8 \%$ versus $5 \%$ ) compared to controls. There was no significant difference between groups for very preterm births (<32 weeks gestation). The risks of microcephaly and neonatal hypoglycaemia were increased in the infants of untreated women with bipolar disorder when compared with the other two groups.

\section{Conclusions/Recommendations}

- Risks of relapse in the immediate postpartum period for women with a history of bipolar disorder or postpartum psychosis are particularly high

- The risk of recurrence appears to be higher if women with bipolar disorder are not taking medication prior to or during pregnancy.

- Bipolar disorder, whether treated or untreated, appears to be related to an increase in preterm delivery, Caesarean section and instrumental delivery. However the strength of this data is poor.

\subsection{Risks related to schizophrenia and other psychotic illnesses}

Overall, pregnancy is not protective against acute or chronic psychosis and there is an increased risk of acute psychotic relapse in the postnatal period though the increased risk is not as high as seen for bipolar disorder (Munk-Olsen et al., 2009). The risk of a postnatal psychotic episode in the context of chronic schizophrenia is evenly elevated throughout the first postnatal year (unlike bipolar disorder, where risk 
rises rapidly in the early days and remains raised for the first few months) (MunkOlsen et al., 2009).

Compared to the general population, women with schizophrenia are more likely to have adverse obstetric and neonatal outcomes (Bennedsen et al., 1999; Bennedsen et al., 2001; Cannon et al., 2002; Dalman et al., 1999; Howard et al., 2003; Jablensky et al., 2005; Vigod et al., 2014). Potential mechanisms for this include the effects of the underlying schizophrenic illness; lifestyle factors (e.g. smoking, alcohol and drug misuse and poor nutrition), medical morbidity (in particular diabetes mellitus, obesity and hypertension); social adversities (e.g. poverty, domestic violence); and poor antenatal care (Abel and Howard, 2014; Jones et al., 2014). It is hard to untangle an additional contribution to adverse outcomes from psychotropic medication (including antipsychotics and other commonly co-prescribed medications such as antidepressants, mood stabilisers and benzodiazepines) given a relative paucity of data from mothers who were ill and untreated. A study of a nationwide population-based dataset in Taiwan found no significant difference in low birth weights or preterm delivery for infants of women with treated or untreated schizophrenia (Lin et al., 2010). However psychotic illnesses are associated with an increased rate of stillbirths and neonatal deaths (Howard, 2005; King-Hele et al., 2009; Webb et al., 2010).

$\underline{\text { Conclusions/Recommendations }}$

- Pregnancy is not protective against episodes of psychosis in women with schizophrenia.

- Schizophrenia is associated with a range of adverse obstetric and neonatal outcomes. The causes of this and the impact of medication is hard to ascertain.

\subsection{Risks of attention deficit hyperactivity disorder (ADHD)}

The challenges of motherhood are likely to be particularly difficult for a woman with ADHD suffering from poor concentration and impulsivity. Untreated or inadequately treated ADHD can result in risk-taking, which may potentially place the mother and child at risk, both prenatally and postnatally. There is also an association 
between ADHD and substance misuse, which may raise additional risks for the fetus and infant (see section 3.9). It is important to note that there is some evidence that adequate treatment of ADHD might decrease substance misuse (Wilens, 2003).

Impulsivity can be a major element of ADHD for some patients. This may increase the risk of unplanned pregnancy (Besag, 2014). This is supported by the observation of higher rates of induced abortions in women with ADHD (Haervig et al., 2014). However this may be confounded by the observation in the same study of women on ADHD medication being younger, more likely to be single, having lower education, being more likely to be receiving benefits, and to be using other psychotropics than other pregnant women.

\subsection{Risks related to eating disorders}

Eating disorders (ED) affect approximately 5-10\% of women of childbearing age (Smink et al., 2012). ED onset is in adolescence and young adulthood (Micali et al., 2013) and usually the disorders have a chronic course.

ED can result in menstrual dysfunction and ovulation problems, difficulties conceiving and an increased need for fertility treatment (Easter et al., 2011; Freizinger et al., 2010; Micali et al., 2014). However, ED are common in the perinatal period, with recent studies estimating the prevalence of active ED in pregnancy between 57.5\% (Bulik et al., 2007; Easter et al., 2013; Micali et al., 2016b). There is some evidence that ED symptoms can improve during pregnancy (Bulik et al., 2007; Easter et al., 2015), however they do not completely disappear and relapse, recurrence and new onset ED occur in the postpartum period (Blais et al., 2000; Crow et al., 2008).

Women with active and past history of ED are at high risk of developing perinatal depression and anxiety (Easter et al., 2015; Micali et al., 2011b). A metaanalysis showed that ED, particularly anorexia nervosa (AN), is also associated with adverse birth outcomes including low birth-weight (around $0.2 \mathrm{Kg}$ less than babies born to non-affected mothers) (Solmi et al., 2014) and babies born small for gestational age $\left(16 \%\right.$ of babies born to mothers with AN below the $10^{\text {th }}$ centile of sex and gestational-specific birthweights) (Micali et al., 2016b). However, opposite 
findings have been reported for women with bulimia nervosa $(\mathrm{BN})$ and binge eating disorder (BED) having babies large for gestational age (Linna et al., 2014).

Conclusions/Recommendations

- ED can reduce fertility but are still common in the perinatal period.

- ED symptoms may improve during pregnancy.

- Women with ED are at increased risk of developing depression and anxiety during pregnancy and postnatally perinatally.

- AN is associated with low infant birth-weights

- $B N$ and BED are associated with infants born large for gestational age.

\subsection{Risks related to substance misuse}

Substance misuse/dependence are important factors in the morbidity and mortality of pregnant women, including suicides. Women who engage with addiction services have better outcomes with better antenatal health care than those women who do not engage. The World Health Organisation (WHO) has published evidence based guidelines on the identification and management of substance use in pregnancy with specific recommendations (World Health Organisation, 2014). Substance use is also a significant factor in the deaths of women in the first year after pregnancy (Knight et al., 2015).

\subsubsection{Smoking/nicotine dependence}

Smoking is the leading preventable cause of fetal morbidity and mortality in the UK. Women with mental disorders are more likely to be smoking at conception, at antenatal booking, and up until delivery (Goodwin et al., 2007) and to find it more difficult to stop smoking, even if they accept referral to smoking cessation services (Howard et al., 2013). Smoking is associated with an increased risk of miscarriage, congenital malformations, low birth weight, prematurity, stillbirths, sudden infant death syndrome and physical and mental disorders in childhood (Royal College of Physicians, 2010). There is good evidence from a large Cochrane systematic review of 77 randomised controlled trials including more than 29,000 individuals that smoking cessation programmes reduce the proportion of pregnant women continuing to smoke, and improve rates of low birth weight and prematurity (Chamberlain et al., 2013). 
The complexity of the relationship between factors influencing the infant's outcome is further illustrated by research investigating the association between maternal smoking during pregnancy and the risk of ADHD in offspring (Langley et al., 2012). This study demonstrated that the risk to the offspring is similar whether it is the mother or father who smoked during pregnancy and this was not as a result of passive smoking on the part of the mother (Langley et al., 2012). This suggests that genetic rather than intrauterine effects may be causal.

\subsubsection{Alcohol misuse/dependence}

Alcohol consumption in pregnancy is associated with miscarriage, preterm birth, low birth weight, and developmental delays encompassed within the umbrella term of Fetal Alcohol Spectrum Disorders (FASD) (Makarechian et al., 1998; O'Leary et al., 2009). Alcohol use in pregnancy is predicted by pre-pregnancy alcohol consumption (i.e., quantity and frequency of typical drinking) and exposure to abuse or violence (Skagerstrom et al., 2011), though there is some inconsistency in the association between antenatal psychiatric disorders or symptoms and alcohol misuse (Lancaster et al., 2010; Skagerstrom et al., 2011).

While alcohol use is key to development of fetal alcohol syndrome, other factors such as other drug use e.g. nicotine and cocaine, stress and malnutrition increase the impact of alcohol use on the fetus and should be considered (Abel and Hannigan, 1995).

\subsubsection{Cannabinoids}

Cannabis is the most commonly used illicit drug, with perinatal prevalence rates up to about $40 \%$ though the majority stop once they know they are pregnant (Forray and Foster, 2015). Cannabis has been associated with adverse outcomes such as low birth weight and preterm labour (Metz and Stickrath, 2015). Cannabis use often occurs with other drugs such as tobacco and alcohol which also have an independent effect on fetal outcomes (e.g. (Kuhn et al., 2000).

\subsubsection{Opioid misuse/dependence}

Opioid dependence is associated with increased maternal and neonatal complications. Untreated opioid dependence is associated with increased risk of fetal growth retardation, placental abruption, fetal death and pre-term delivery (Center for Substance Abuse Treatment, 2008). 


\subsubsection{Stimulant misuse/dependence}

The misuse of amphetamines, cocaine and stimulant-type novel psychoactive substances (NPS) is a public health problem presenting with medical, psychiatric, legal and socio-economic consequences. Cocaine and other stimulant use during pregnancy has been associated with preterm labour, congenital anomalies, intrauterine growth retardation (IUGR), placental abruption, low-birth-weight infants, neonatal death, and sudden infant death syndrome (SIDS) (Debooy et al., 1993; Fox, 1994; Ryan et al., 1987). Maternal complications include a pre-eclampsia-like syndrome, acute pulmonary oedema, seizures, cardiac arrhythmia, and sudden death (Fox, 1994; Ryan et al., 1987). Prenatal cocaine exposure has also been associated with developmental problems amongst pre-school and school age children although there are often multiple confounding environmental variables at play (Ackerman et al., 2010; Frank et al., 2001).

Stimulant use prior to delivery is associated with a neonatal withdrawal syndrome including symptoms of agitation, vomiting and tachypnoea (Chomchai et al., 2004; Smith et al., 2003).

\subsubsection{Benzodiazepine misuse/dependence}

These drugs may be a significant problem in pregnant women, often taken with opioids. In one study of pregnant opioid dependent women, $44 \%$ of women screened were positive for benzodiazepine misuse (Jones et al., 2010). See section 5.2. Conclusions/Recommendations

- Smoking in pregnancy is associated with a range of adverse pregnancy and infant outcomes and it is recommended that health professionals refer to pregnancy smoking cessation services and support women with engagement with smoking cessation and quit attempts.

- Alcohol is associated with serious adverse effects including fetal alcohol syndrome.

- Illicit drug use is associated with a range of adverse fetal and maternal outcomes. 


\section{USING MEDICATION IN THE PERINATAL PERIOD}

The management of mental illness in the perinatal period is an area so devoid of evidence in many areas, and with risks which are hard to quantify, that the most important element of this document are the general principles. One of the most important of these is collaboration between the prescriber and the woman, and potentially her partner.

\subsection{Weaknesse in the data on which perinatal guidelines are based}

Historically much of the data regarding the risks of psychotropics in the perinatal period has been retrospective, with all of the associated biases that this entails. Malformations following exposure are more likely to be reported than healthy outcomes due to increased vigilance in women taking medication. This also applies to population datasets. Increased screening for malformations amongst infants exposed in utero to a drug can lead to increased detection, including of minor malformations that are of no clinical significance. The literature does not always differentiate between minor clinically insignificant malformations and more serious ones, or reports them together. Some data come from studies of the use of drugs for nonpsychiatric indications, and therefore in populations with potentially different vulnerabilities (e.g. the use of anticonvulsants in epilepsy). However in recent years, at least for antidepressants, there have been several cohort studies, particularly from Scandinavia, that have reported on large number of exposures in pregnancy (in excess of 100,000). Even with this amount of data it can be difficult to draw conclusions about the reproductive safety of these medications.

There are two major issues that need to be considered when reviewing the literature. Firstly there are residual confounding issues such as coexisting alcohol use and smoking, as well as the severity and type of psychiatric illness that the mother herself is suffering from. Secondly there is an issue relating to multiple statistical tests that are undertaken in some studies exploring the effects of medication on a range of factors. For example, a Swedish study of the effects of in utero exposure to antidepressants tested multiple comparisons by including many outcomes, 21 different drugs and the impact of exposure at multiple time points in pregnancy (Reis and Kallen, 2010). 
The evidence we have on efficacy for most medications specifically in the perinatal period are also poor. Most RCTs of psychotropics exclude women who are pregnant or breastfeeding. In general, guidelines (e.g. (National Institute for Health and Care Excellence, 2015a; Scottish Intercollegiate Guidelines Network, 2012)) have extrapolated efficacy findings from non-pregnant and non-breastfeeding women and men. This seems reasonable as there is little evidence to suggest that the efficacy of psychotropics could be different in the perinatal period.

\section{Conclusions/Recommendations}

- Data regarding the risks and benefits of psychotropic medication in the perinatal period has many weaknesses, in particular the multiple confounding factors. This makes definitive statements about risks and benefits impossible in most cases.

- There is relatively little data regarding the efficacy of psychotropics specifically in pregnancy and breastfeeding women. It is therefore usually necessary to generalise evidence of efficacy from studies in non-perinatal populations.

\subsection{Recommended principles of prescribing to women in the perinatal period}

\subsubsection{General management issues across all childbearing stages}

- Factors influencing management choices in the perinatal period include:

○ Accuracy of the diagnosis.

- Illness course, severity, burden and risk when unwell.

- Presence of psychiatric and physical comorbidities.

- Frequency of, and triggers for, relapse.

- Previous perinatal episodes of illness in the women and family history of severe perinatal episodes.

- The woman's treatment history including her responses and tolerability to medication, talking therapy and other interventions and her experience of them.

- Past episodes of self-harm, suicide attempts and risks to others.

- Duration of remissions on and off medication. 
- Social functioning.

- Time to relapse after previous discontinuation.

- Time to recovery on reintroduction of medication.

- Check whether the woman and her family have been sufficiently educated about her illness and explain how childbearing may affect its course.

- Consider the attitudes of the woman and her partner to the balance of risk of recurrence of illness and reproductive safety of medication.

- After a careful consideration of the above factors, it may be that a joint decision is to utilise non-drug interventions as alternatives to medication where appropriate.

- For women on several medications, take a careful history of the indications for each prescribed medication and its effectiveness, and minimise the number and dose of medications as far as possible.

- Using a drug of known efficacy in a particular woman may be preferable to using one of unknown efficacy but possible lower pregnancy risk.

- Sub-therapeutic doses of medication should be avoided.

- Explore possible substance use/misuse and address this where possible.

- Because of the high risk of a significant recurrence in the perinatal period and the complexities of good care, women with history of severe mental illness should be under the care of mental health services, ideally a specialist perinatal mental health team wherever possible.

- All women with significant histories of severe mental illnesses should have an individualised perinatal mental health care plan drawn up in collaboration with the woman herself and mental health, primary care, health visitors, maternity services, neonatology and Children's Social Care if involved. Such care plans should take into account and describe:

- The woman's obstetric, physical and mental health care needs

○ Ongoing medication and psychosocial interventions and steps to address relapse prevention.

- Information about any relevant monitoring issues (e.g. of lithium levels), medication dose adjustments, interactions with other drugs that 
could be prescribed in the pregnancy, any relevant issues related to delivery and possible risks to the neonate.

- Detailed, individualised letters recording discussions and decisions should be written to women and copied to all professionals involved.

- The specified roles for each professional in terms of monitoring, providing interventions and co-ordinating care.

- Whether or not the woman plans to breast feed and any medication plans for this period of time.

- Whether there are any relevant safe guarding issues.

- The care plans should be distributed widely amongst all involved health professionals.

\subsubsection{The preconception period}

- There should be discussions with all women of child bearing potential who are being prescribed psychotropics or with a history of mental illness regarding their risks and benefits related to conception and during the perinatal period, as well as options for contraception. For women with a history of mental illness considering starting or extending their family more formal extended preconception counselling is required, reviewing the women's current health and the available evidence of fetal risk for any drugs she is taking.

- Pre-conception advice is particularly important where medication is associated with early teratogenicity.

- There is a particular concern around the use of anticonvulsant mood stabilisers, such as valproate or carbamazepine, whose adverse effects may have occurred before confirmation of pregnancy.

- Valproate is the only psychotropic contraindicated in women of childbearing potential when used for psychiatric indications, although even here there can be very rare exceptions.

- Options available to women include staying on current regimes, switching to a regime that may be associated with fewer adverse fetal and/or maternal effects, or discontinuing treatment entirely. 
- If a decision is taken to discontinue medication, this may need to be done slowly, for example, to avoid withdrawal symptoms or to enable monitoring for signs of maternal deterioration. The interval following discontinuation during which effective contraception needs to be continued will depend on the half-life and teratogenic risk associated with the drug.

\subsubsection{During pregnancy}

- Manage pregnancy among women with severe mental illness as a high risk pregnancy requiring more intensive monitoring, with close liaison among professionals (general adult and specialist perinatal mental health professionals, obstetricians, midwives, GPs and social services where appropriate).

- Addressing any modifiable risk factors at baseline and throughout the perinatal period: smoking (smoking cessation programmes), obesity (behavioural interventions and consider prescribing options), nutritional status (folate supplementation), diabetes mellitus (screen and treat), alcohol and drug misuse (refer to specialist services if needed) and domestic violence (refer to advocacy / voluntary sector specialist services).

- Risk/benefit decisions about staying on medication or discontinuing should be considered as soon as possible on discovering a pregnancy and individualised.

- Generally avoid stopping medication suddenly on discovery of pregnancy since this does not necessarily remove risks of malformations and sudden discontinuation of medication may pose risks to the mother's health.

- There is a different risk/benefit analysis to be made when continuing/changing pre-existing medication, compared with commencing a new treatment.

- The most important information regarding the likely efficacy of a medication for a particular woman comes from the patient's own past treatment history. If there is strong evidence of a good past response to a particular medication, there should be a good reason not to recommend it as the medication of choice or to prescribe a medication of unknown efficacy even if with a potentially lower risk in pregnancy. 
- In general, avoid switching medication in pregnancy unless the benefits are likely to outweigh the risks.

- Women taking psychotropics during pregnancy that are known to be associated with a significant risk of malformations should be offered detailed ultrasound scanning for fetal abnormalities as early in gestation as such scanning is possible, if they choose.

- Monitor for excessive weight gain, and ensure this is managed in line with appropriate guidance (e.g. (National Institute for Health and Care Excellence, 2010c)). Also monitor for gestational diabetes, particularly for women on a second generation antipsychotic (SGA), (request an oral glucose tolerance test) and ensure this is managed in line with appropriate guidance (e.g. (National Institute for Health and Care Excellence, 2015b)).

- Considering high dose folic acid (5mg/d) where folate-lowering drugs are used, although the evidence for its benefit has not been shown (Wlodarczyk et al., 2012) and there is a possibility that folate may decrease the efficacy of other drugs such as lamotrigine in bipolar disorder (Geddes et al., 2016).

- While there may be some potential advantages of changing medications early in pregnancy, by mid-trimester there might be fewer, if any, advantages in changing (with the exception of valproate).

- In later pregnancy, there is also a risk/benefit decision to be considered around risk of neonatal adaption syndromes and e.g. persistent pulmonary hypertension of the newborn (PPHN - see section 5.1.3).

- Close to delivery, there needs to be consideration of whether there is any evidence that reduction/discontinuation of medication will alter the risk of neonatal syndromes particular to the medication in question, taking into account the risks to the mother of doing so.

- For most women, the delivery date is not certain so the woman may be exposed to an ineffective dose for several weeks at a time when risks (eg of bipolar relapse) may begin to increase.

- There is no evidence on how long reduction/discontinuation would need to be for to reduce this risk, but 14 days may not be enough (Warburton et al., 2010). 
- Plans to breastfeed should also be taken into account as this may mitigate discontinuation.

- As neonatal adaption syndromes are generally mild and transient, this issue is probably most relevant in pregnancies with known fetal problems such as intrauterine growth retardation or malformations.

- During an admission to the maternity unit, consider the need for a specialist mental health nurse or an advocate where appropriate.

\subsubsection{The intrapartum period}

- In women with a severe mental illness, it is recommended that delivery should be in hospital.

- Anticipate potential issues which might arise.

- Midwives need to be aware of any medication issues, for example the need to stop lithium when the woman goes into labour, or any medication that might need to be started immediately following delivery, for example in a woman at risk of a postnatal episode.

- Information needs to be provided as to when and how clinical staff on the maternity ward should seek psychiatric advice.

- Information about potential risks of medication taken during pregnancy on the baby needs to be discussed with the relevant neonatologists.

\subsubsection{Postnatally and during breast feeding}

- The World Health Organisation recommends that infants should be exclusively breastfed for the first six months of life to achieve optimal growth, development and health' (WHO, 2003).

- Take care with any sedating medication especially in the postnatal period since excessive sedation can hinder baby care and breastfeeding. While sedation can often resolve over a short period after starting a medication, alternative options may need to be considered.

- Advise women against co-sleeping with their babies.

- Where relevant, consider a discussion about the woman's parenting capacity and the potential need for referral to Children and Families social services. Where a woman has significantly impaired social function and poor social support, consider discussing the risk of custody loss. 
- Breastfeeding women with mental illness should have rapid access to psychological therapies, but where illness is more severe, drug therapy may be an essential component of effective treatment.

- Choose treatments for women who are breastfeeding with the lowest known risk and keep in mind that there is very limited knowledge about long term neurodevelopmental outcomes.

- A guide to the possible risk of breast feeding while taking a particular drug is the relative infant dose (RID) - the daily amount of drug ingested by the infant during exclusive breastfeeding per $\mathrm{kg}$ bodyweight divided by the maternal daily dose per $\mathrm{kg}$ body weight. Information regarding this can be found in the Drugs and Lactation Database, 2016 (https://www.nlm.nih.gov/pubs/factsheets/lactmedfs.html).

- As in pregnancy, previous treatment response should guide future treatment choices.

- Where there is no clear evidence base that one drug is safer during breastfeeding than another, the best option may be not to switch.

- There is little clear evidence to support the discarding of breastmilk or timing of breastfeeding in relation to time of maternal drug administration. Such recommendations could potentially add to the difficulties and challenges of establishing breastfeeding.

- Monitor the infant for adverse effects such as (but not limited to) over sedation and poor feeding.

- These recommendations in general apply to term, healthy infants. Great caution should be exercised in decisions around maternal psychotropic prescribing for breastfed premature or sick infants, and where the mother is taking more than one drug.

\subsection{Recommendations regarding discussion of the risks and benefits of psychotropics with women}

- Some degree of discussion is required for all women of childbearing potential being prescribed a psychotropic. 
- For women who are considering starting or extending their families the need for more extensive preconception counselling should be considered, ideally to be delivered by specialist perinatal mental health services for women with the highest or most severe risks (history of bipolar disorder or severe mental illness).

- The main goal of discussions is to individualise the advice to the woman taking into consideration her particular history of illness, response to treatment, and attitudes to risk both to her and to the baby.

- See section 2.1 for a framework described by NICE of the various risks and benefits that need to be considered in the discussions.

- As discussed in section 3, women should also be aware not only of the known or potential risks of medication but also of the evidence that untreated illness in pregnancy may be associated with increased adverse effects for the woman herself, her pregnancy, and for infant outcomes.

- Consideration should be given to providing information in a number of ways, for example in both verbal and written forms.

- There are a number of online resources (e.g., www.medicinesinpregnancy.org from UKTIS) that provide evidence based and updated information to assist women in making informed decisions about treatment, although these do not remove the need for detailed individualised assessment and discussion of options with a clinician.

- The nature and level of discussion regarding the risks and benefits of psychotropics in the perinatal period should be tailored to the needs of the woman and, where relevant and agreed by the woman, her partner.

- Women may be concerned about the risk that any children they have may suffer from mental illness. It is therefore important to discuss this issue and consider referral for more formal genetic counselling if available.

- Discussions should consider the risks of exposure both in utero and via breastfeeding on the child in relation to birth defects, neonatal complications 
and developmental or longer term effects. However, the benefits of medication should also be considered in relation to the potential for treating the mother's current mental illness and/or preventing a relapse, acknowledging the impact such illness can have on the child as well as the mother and wider family.

- Risks, wherever possible, should be individualised. In this regard care should be taken in recommending switching a woman from a drug with known adverse effects in the perinatal period to one with perceived lower risk (which may in fact reflect a lack of data rather than evidence of safety), especially if there is evidence of efficacy of the former, but not the later, drug in the woman concerned.

- The degree of risk is uncertain in relation to many of the elements that need to be considered when discussing the use of psychotropics in the perinatal period. It is important to explicitly acknowledge this uncertainty with women and their partners. Simply describing the size of the risk without putting this into the context of absolute risk rates can be misleading.

- Care should be taken to avoid medical jargon when discussing risks and benefits. Care should also be taken that the biases of the clinician does not colour the description of the relevant risks and benefits of the various options.

- Be aware that a woman may also be receiving information from multiple professionals and significant others, or have read out-dated or inaccurate information on the internet.

- Women should be afforded the opportunity of discussing the issues around the use of psychotropics in the perinatal period broken down over more than one session, and again at any significant point of potential change in management.

- Clinicians should carefully document a record of discussions regarding the use of psychotropics during the perinatal period. This is best done by providing the woman with a letter detailing in non technical language, the discussion and conclusions reached, and copying this to all professionals involved, so that the record becomes a clinically and educationally useful, shared one. 
- In any discussion of potential adverse outcomes, the potential for inducing or exacerbating excessive worry or inappropriate guilt must be considered.

\section{BENEFITS AND HARMS ASSOCIATED WITH INDIVIDUAL MEDICATIONS}

The effects of a medications on the ability to conceive, or on a pregnancy and its outcome (e.g. miscarriage, live birth, still birth or intrauterine death), and on the developing fetus, are reviewed below and should always be taken into account when prescribing in pregnancy or to women who wish to breastfeed.

\subsection{Antidepressants}

Exposure to antidepressants in pregnancy has increased over recent decades, with a registry study from Denmark reporting a 16 fold increase from $0.2 \%$ of pregnancies in 1997 to $3.2 \%$ in 2010 (Kjaersgaard et al., 2013).

Although there are many large studies examining the reproductive safety of these drugs, with one recent study including 128,950 exposures (Huybrechts et al., 2015), any specific impact of antidepressants independent of maternal depression is still unclear.

Most recent, large scale, research focusses on SSRIs. The popular belief that tricyclics may be safer than SSRIs was challenged by a large study by Reis and Kallen (2010), demonstrating that outcomes after exposure to tricyclics (albeit predominantly clomipramine) are, for most measures (preterm birth, low birth weight, hypoglycaemia, respiratory diagnoses, low Apgar score, jaundice and cardiac malformations), not significantly different than after exposure to SSRIs or other antidepressants (Reis and Kallen, 2010). It is important to note however that differences in the nature of the women (and their depression) who are prescribed a tricyclic versus an SSRI may confound this finding. In addition, not all studies address the confounder effects of depression per se, leaving the conclusions on the direct adverse consequences uniquely attributable to antidepressants still unclear.

Given that guidelines tend to recommend SSRIs as first line drug treatments for depression (National Institute for Health and Care Excellence, 2009), including in pregnancy in antidepressant naïve women (National Institute for Health and Care 
Excellence, 2015a), these will largely be the focus of this section (note bupropion is covered in section 5.7.1).

\subsubsection{Effects on ability to conceive}

There are no data suggesting antidepressants impact on the ability of women to conceive, although there is some evidence that they may affect male sperm count and morphology (Akasheh et al., 2014).

\subsubsection{Pregnancy outcomes}

A recent review summarised the literature relating to antidepressant impact on pregnancy outcome (Ross et al., 2013) and while including 23 studies, it was notable that only 6 studies made any attempt to control for depression. It found no significant impact of $\mathrm{AD}$ exposure on rates of spontaneous abortion; a small impact on gestational age at delivery (0.45 weeks earlier); a modest impact on Apgar scores (0.37 lower at $1 \mathrm{~min}, 0.18$ at 5 mins); and a modest impact on birth weight $(74 \mathrm{~g}$ lower), although this last was not significant if the comparison group was women with depression. However, there is conflicting data and not all antidepressants may be associated with the same magnitude of risk.

Drugs that block serotonin uptake, such as many antidepressants, are associated with increased risks of bleeding (National Institute for Health and Care Excellence, 2009). There have been reports of particularly third trimester exposure to antidepressants being associated with an increased risk of postpartum haemorrhage (Bruning et al., 2015; Hanley et al., 2016). However, the data are limited and the magnitude and clinical significance of the risk uncertain.

\subsubsection{Teratogenicity}

Studies first reported an increased risk of cardiac defects with paroxetine exposure in 2006 (GSK, 2006 http://www.gsk.com/media/resourcecentre/paroxetine/paroxetine-and-pregnancy.html; (Kallen and Otterblad, 2007), indicating an absolute risk for cardiac defects of around $1.5 \%$ to $2 \%$ in babies born to mothers who took paroxetine compared with a rate of around $1 \%$ in the general population. Subsequent studies have implicated other antidepressants, including all commonly used SSRIs and clomipramine (Reis and Kallen, 2010; Shen et al., 2016). Another large study that adjusted for confounding factors found that small but significantly increased risks of cardiac malformations with fluoxetine (ventral septal defects) and paroxetine (right ventricular outflow tract defects), were no longer 
significant after adjustment for depression-associated confounders, such as smoking, other psychiatric medication, and fetal alcohol syndrome (Malm et al., 2011). Similarly a very large study that also adjusted for depression-associated confounders found no substantial increase in the risk of cardiac malformations (Huybrechts et al., 2014a).

A specific problem that has been associated with serotonergic antidepressant use in mid to late pregnancy is persistent pulmonary hypertention of the newborn (PPHN), a severe condition with a high mortality. While initial reports suggested an approximate 6-fold increase, equating to 6-12/1000 births to SSRI exposed pregnancies compared to $1-2 / 1000$ births in the general population (Chambers et al., 2006), a recent study combining data from a number of Scandinavian registries found the absolute risk to be small and more modest (approximate 1.5 fold increase from around 20 per 10,000 births to around 30) and this was no longer significant when confounders were taken into account (Huybrechts et al., 2015).

In general, for all adverse outcomes, studies which have taken account of the underlying depression and other confounding factors, find smaller effects potentially attributable to antidepressant medication.

\subsubsection{Neonatal and developmental problems}

The data appear to support the existence of a neonatal behavioural syndrome following in utero exposure to antidepressants, including irritability, constant crying, jitteriness, vomiting, shivering, increased tone, and eating and sleeping difficulties. Moses-Kolko and colleagues (2005) identified 9 cohort studies, 5 of which defined a neonatal behavioural syndrome, and found it occurs three times more often in neonates exposed to SSRIs in late pregnancy (30\% in SSRI exposed, $10 \%$ in general population) (Moses-Kolko et al., 2005). They also found that admission to a special care baby unit (SCBU) was 2-3 times more common in the SSRI exposed neonates, although it is possible that this may be a precaution in some cases to enable monitoring rather than being indicative of symptoms. A recent meta-analysis of 12 studies found a significant 5-fold increased risk (5-78\% risk in exposed vs 0-41\% in non-exposed infants) of postnatal adaption syndrome or respiratory distress and tremors in infants exposed to antidepressants before delivery (Grigoriadis et al., 2013). However, as per the evidence for teratogenicity, there is also the possibility that these adverse neonatal outcomes are not due to antidepressants toxicity or withdrawal 
but rather to the underlying maternal disorder or concurrent exposures. One naturalistic observation study compared the outcomes of neonates exposed to SSRIs in the last 14 days of pregnancy and neonates exposed in pregnancy but not in the last two weeks, and found an increased risk of neonatal respiratory distress in the lastexposed group (Warburton et al., 2010). However, when controlled for maternal illness severity, reducing exposure to SSRIs at the end of pregnancy did not improve neonatal health.

Data on long-term effects of antidepressant exposure in utero are particularly sparse. One study of the Danish National Birth Cohort, which compared women on antidepressants, women with depression in pregnancy who did not take antidepressants, and women with no depression or antidepressant use, found a small developmental delay in gross motor function related to medication in late pregnancy, however, there were no differences between groups by 19 months (Pedersen et al., 2010).

There have been a number of studies that have suggested a relationship between in utero antidepressant exposure and both ADHD and ASD with suggestions of around a two-fold increased rate of ASD from a background rate of approximately 0.7-1\% (Boukhris et al., 2016; Croen et al., 2011; Figueroa, 2010; Man et al., 2015). However, there have also been large cohort studies which failed to find such associations (Castro et al., 2016; Laugesen et al., 2013). The discrepancy may be due to potential confounding of the maternal indication for taking antidepressant in that while some studies have still found an association (albeit reduced in magnitude) after controlling for maternal depression (Boukhris et al., 2016), in others the association is no longer significant (Clements et al., 2015). This explanation is also supported by two other observations. The first is that in a large Finnish cohort study, prenatal exposure to SSRIs was associated with an increased risk of depression (8\% vs $2 \%$ in non-exposed children), but not ASD or ADHD, in early adolescence. A potential, at least partial, explanation for this observation is shared genetic risk factors. The second observation is that a meta-analysis has suggested that there is a significant increased risk of ASD in children whose mothers were exposed pre-conception to SSRIs (Kaplan et al., 2016). While there may still be a plausible biological mechanism for pre-conception maternal exposure to lead to a developmental problem in their offspring, as the authors of the meta-analysis point out, confounders by 
indication for the SSRI, including shared genetic risk, cannot be ruled out. Taken all together, there is uncertainty about whether in utero exposure increases the risk of ASD or ADHD and reported findings may well result from confounding factors.

More reassuringly, at least one study has indicated potential long term benefit to the children of mothers with depression in pregnancy being treated with antidepressant medication. Data from 49,000 women examined the impact of prenatal exposure to SSRIs on behavioural problems at aged 7, finding that untreated prenatal depression was associated with an increased risk of problem behaviour in the children (hyperactivity, inattention, and peer problems) but, crucially, this increased risk was not seen in the children whose mothers took antidepressants in pregnancy (Grzeskowiak et al., 2015). This study confirms the abundant evidence that maternal depression in pregnancy increases the risk of psychopathology and other social adversity in the offspring into adolescence and young adulthood (Plant et al., 2015b), and supports previous assumptions that treating depression reduces this risk.

\subsubsection{Breastfeeding}

Most evidence regarding the safety of antidepressants during breastfeeding comes from single case reports and case series, only a minority of which include a control population. Where larger samples are reported, they usually include a mix of exposed drugs, and indirect measures of infant drug exposure, if measured at all. Moreover, studies of breastfed infants are often confounded by antenatal exposure to the same drug, which may act as a 'loading dose', increasing the risk of early adverse effects. Few studies report on depression-associated confounders, such as maternal smoking, alcohol or substance use, or on infant characteristics, such as weight. Lastly, there is a paucity of studies examining the long-term effects of drug exposure through breast milk on infant development. Where this has been reported, it is usually only within the first 12 months of life.

Overall, SSRIs with lowest reported levels of exposure or adverse physical effects include sertraline and paroxetine (Berle and Spigset, 2011; Davanzo et al., 2011; Orsolini and Bellantuono, 2015). Given the greater risks of side effects and withdrawal problems with paroxetine compared with other SSRIs (Cleare et al., 2015), sertraline would be the preferred treatment option. 


\section{Conclusions/Recommendations}

- The majority of data on the effects of antidepressants are confounded by many uncontrolled variables, including the impact of depression itself.

- Antidepressants, when studied as a group, may have a small effect on some pregnancy outcomes (gestational age, Apgar score) but these may be due to residual confounding issues and in addition may not be clinically significant.

- Antidepressants that block serotonin uptake may be associated with an increased risk of postpartum haemorrhage, but the magnitude and clinical significance of this risk is uncertain.

- There have been concerns about antidepressant (mainly SSRIs and possibly particularly paroxetine) exposure in utero being associated with cardiac malformations. However this may not be the case once all confounders are taken into account.

- Although the risk of PPHN in babies exposed in utero to SSRIs is increased, the absolute risk is low.

- There is some evidence of neonatal effects following late pregnancy exposure to SSRIs, particularly respiratory distress and neonatal behavioural syndrome.

- Some studies have reported an association between exposure to antidepressants in utero and an increased risk of ASD or ADHD later in childhood. However the data are mixed with other studies suggesting the association may be due to confounding.

- Babies born to mothers with depression and treated with antidepressants during pregnancy may have better longer term emotional and behavioural development compared to babies born to mothers with depression that was untreated.

- Sertraline has a low rate of reported adverse effects on breast fed babies and for new episodes of depression may be appropriate. However the choice of antidepressant should be guided by the same principles as choice during pregnancy, for example taking into account a woman's previous response to medication. Careful monitoring of the infant is always important. 
- Recommendations regarding the use of antidepressants in depression and anxiety are described in sections 6.1 and 6.2 respectively.

\subsection{Anxiolytics/Hypnotics}

This section considers benzodiazepines, "z-drugs" (zolpidem, zopiclone and zaleplon), buspirone, beta-blockers, pregabalin and gabapentin.

There is much uncertainty about the effects of anxiolytic or hypnotic medications on fertility, in pregnant or breastfeeding mothers, or on subsequent infantile and childhood development. A systematic review (including 43 controlled studies) of possible associations between maternal antidepressant or anxiolytic drug treatment and childhood neurodevelopmental measures emphasised the inconsistent nature of individual study findings and stresses the need for further well-designed observational studies (El et al., 2014). Much of the reported literature does not control for important confounders such as the type and severity of maternal illness.

\subsubsection{Effects on ability to conceive}

There is no human data in either men or women regarding the effects of anxiolytic and hypnotic drugs on fertility.

\subsubsection{Pregnancy outcomes}

Beta-blockers cross the placental barrier and can increase insulin levels and decrease glucagon levels, with a consequent risk of hypoglycaemia in new-born infants (Bricelj, 1999). A pharmaco-epidemiological study found that infants exposed to beta-blockers in the third trimester of pregnancy had an approximately three-fold risk of hypoglycaemia ( $8 \%$ vs $2 \%$ risk in unexposed infants) and two-fold risk of feeding problems (9\% vs 5\%) (Davis et al., 2011). Additionally, a Danish population study found beta-blocker exposure in pregnancy to be associated with around a twofold increased risk of 'small for gestational age' (18\% vs $10 \%$ for unexposed infants), pre-term birth ( $28 \%$ vs $12 \%$ ) or perinatal mortality (1.8\% vs $0.7 \%$ ) (Meidahl et al., 2012). However, because maternal hypertension itself increases the risk of intrauterine growth restriction (Lin et al., 2015), causal analysis is complex and any contribution of beta-blocker exposure to these outcomes remains unquantified. Overall, the available data do not suggest that gestational beta-blocker exposure increases the risk of preterm delivery (Magee and Duley, 2003). Data on rates of 
spontaneous abortion, stillbirth and neurodevelopmental outcomes are too limited to permit a risk assessment.

Zolpidem crosses the placental barrier (Juric et al., 2009). A population based study from Taiwan of 2,497 mothers taking zolpidem during pregnancy compared with 12,485 mothers on no medication found a 40-70\% increased risk of pre-term delivery (10\% vs $6 \%$ for unexposed infants), low birth weight (8\% vs 5\%) and Caesarean section (47\% vs 33\%) (Wang et al., 2010). However, there was no adjustment for the indication for which the zolpidem was being prescribed.

There are little other data on the effects of anxiolytics and hypnotics on pregnancy outcomes.

\subsubsection{Teratogenicity}

Retrospective case reports and cohort studies have previously found an association between benzodiazepine exposure and oral cleft (Dolovich et al., 1998). However this finding has not been replicated in prospective cohort studies (Wikner et al., 2007). Additionally a UK population-based cohort study suggests first trimester exposure to benzodiazepines or non-benzodiazepine hypnotics is not associated with an increase in major congenital anomalies (Ban et al., 2014).

Little is known about the safety of gabapentin or pregabalin in pregnant women, but both drugs are eliminated unchanged in urine and their plasma levels should be expected to decline during pregnancy (Reimers, 2014). A Norwegian birth registry study detected no increase in the rate of congenital malformations among infants of women exposed to pregabalin during pregnancy (and no reported malformations in infants exposed to gabapentin), when compared to a control group of woman with epilepsy who were not undergoing anticonvulsant treatment (Veiby et al., 2014). However, an increased risk of major malformation was associated with pregabalin exposure in a recent prospective cohort study though the small number of exposed pregnancies and potential confounding may account for the findings (Winterfeld et al., 2016).

There are no human data regarding the safety of buspirone in pregnant women.

Information is limited about the safety of so-called 'Z-drugs' in pregnant women though a national birth registry study indicates that in utero exposure to zopiclone, zolpidem or zaleplon is not associated with an increased risk of congenital malformations (Wikner and Kallen, 2011). 
A meta-analysis has suggested that gestational beta-blocker exposure may be linked to increased risks of cleft lip and/or palate and neural tube defects in the infant (Yakoob et al., 2013), although these findings remain to be confirmed. This review also suggested a possible increased risk of congenital heart defects amongst infants of women who were treated with beta-blockers in pregnancy (Yakoob et al., 2013). However, increased rates of cardiovascular malformation have been observed with other antihypertensive therapies (Caton et al., 2009). This association may therefore reflect a physiological effect of lowering the maternal blood pressure rather than a drug-specific teratogenic effect, or may be causally related to the underlying maternal hypertension (Ramakrishnan et al., 2015).

\subsubsection{Neonatal and developmental problems}

Little is known about the effects of in utero exposure to anxiolytic drugs on infantile development. An early longitudinal case-control study suggested that exposure to meprobamate or chlordiazepoxide $(n=1870)$ had no adverse effects (Hartz et al., 1975), but a small $(\mathrm{n}=17)$ follow-up study of children exposed to various benzodiazepines suggested early (up to 18 months) motor and wider developmental delay (Laegreid et al., 1992; Viggedal et al., 1993). A more recent Danish regional prescription register study suggested in utero benzodiazepine (and antidepressant and antipsychotic) exposure is possibly associated with an increased risk of delayed psychomotor development (Mortensen et al., 2003). This is consistent with a longitudinal study $(n=184)$ which suggests that exposure to concomitant benzodiazepines during antidepressant treatment in pregnant women may increase the risks of lower motor scores, higher 'CNS stress signs' across the first postnatal month, and of lower self-regulation and higher arousal in the first two weeks after delivery (Salisbury et al., 2016).

Use of beta-blockers near term may result in neonatal beta-adrenoceptor blockade leading to neonatal bradycardia, hypotension and hypoglycaemia (Bateman et al., 2016). Neonatal respiratory distress has also been reported (Magee and Duley, 2003).

\subsubsection{Breastfeeding}

Potential consequences of benzodiazepine administration in breastfeeding mothers are not clearly established but a prospective cohort study suggests neonatal adverse events are uncommon (1.6\% of infants) (Kelly et al., 2012). However 
analysis of a French pharmacovigilance database led the authors to suggest benzodiazepines should be used cautiously in breastfeeding mothers (Soussan et al., 2014). Potential adverse consequences vary between drugs (Newport et al., 2008). Flimsy evidence suggests diazepam and chlordiazepoxide may be preferable to other benzodiazepines (Ito et al., 1993).

Gabapentin levels in breast milk are similar to those in maternal plasma (Reimers, 2014), which might suggest that close monitoring of babies breastfed by mothers taking gabapentin is advisable.

A small study suggests zaleplon crosses into breast milk but only in small quantities, and in rapidly declining levels after single-dose administration (Darwish et al., 1999).

\section{Conclusions/Recommendations}

- There are very limited data pertaining to fertility effects of anxiolytics/hypnotics in humans.

- Zolpidem may increase the risk of adverse pregnancy outcomes including preterm delivery and low birth weight of infants, though the magnitude of this risk if it exists is uncertain.

- There is no convincing evidence in the available data of an increased risk of birth defects associated with benzodiazepines or 'z-drugs'.

- There is uncertainty whether benzodiazepine exposure in utero may be associated with delayed psychomotor development.

- Beta-blocker exposure in pregnancy has been associated with a number of possible risks, though the data may be confounded by the maternal indication for the treatment.

- There are very limited data regarding the risks of gabapentin, pregabalin and buspirone exposure in utero.

- Hypnotics and anxiolytics cross into breast milk so close monitoring of babies is required in mothers who are breastfeeding and taking these medications.

- Recommendations around the use of anxiolytics and hypnotics is described in section 6.2 


\subsection{Antipsychotics}

Antipsychotics are increasingly prescribed in pregnancy; most commonly for depression, followed by bipolar disorder and schizophrenia (Petersen et al., 2016; Toh et al., 2013). The summary below is based on evidence from systematic reviews (Coughlin et al., 2015; Gentile, 2010; National Institute for Health and Care Excellence, 2015a; Terrana et al., 2015) and more recent original studies (Cohen et al., 2016; Petersen et al., 2016; Vigod et al., 2015). Studies up to 2016 included a total of around 4000 women exposed to a range of antipsychotics in pregnancy, and the evidence was significantly expanded in 2016 with the publication of a large US study on congenital malformations among more than 9000 women exposed to atypical antipsychotics and 700 women exposed to typical antipsychotics (Huybrechts et al., 2016).

Women who take antipsychotics in pregnancy are more likely to be obese, smoke, drink alcohol, use drugs, use other medications and have pre-existing diabetes and hypertension than pregnant women in the general population (National Institute for Health and Care Excellence, 2015a; Petersen et al., 2016; Vigod et al., 2015). In general, studies that compare women exposed to antipsychotics in pregnancy to healthy unexposed women report moderately increased risks for several adverse maternal and infant outcomes (including congenital malformations, pre-term birth, fetal growth abnormalities and poor neonatal adaptation) (Boden et al., 2012b; Habermann et al., 2013; Reis and Kallen, 2008; Sadowski et al., 2013). By contrast, the few studies that used a comparison group of women with psychiatric illness but unexposed to antipsychotics (Boden et al., 2012a; Lin et al., 2010; National Institute for Health and Care Excellence, 2015a; Petersen et al., 2016), or studies that adequately controlled for confounding (Vigod et al., 2015), report few associations between antipsychotics and adverse maternal or infant outcomes. Overall, there is little difference in fetal risks between first and second generation antipsychotics (FGA and SGAs), or between individual antipsychotics, but human pregnancy safety data for some individual antipsychotics are limited. In utero exposure data are most available for olanzapine and quetiapine (more than 800 exposed cases each) followed by risperidone and haloperidol (around 400 exposed cases each), with more limited data for clozapine and aripiprazole (Habermann et al., 2013; Larsen et al., 2015; National Institute for Health and Care Excellence, 2015a; Vigod et al., 2015). It should 
however be noted that not all exposures occurred in the first trimester and evidence to support a lack of structural teratogenesis or increased risk of miscarriage may therefore be less strong.

Placental passage of antipsychotics is incomplete but varies between drugs. Amongst four antipsychotics studied in one sample, highest rates were found for Olanzapine, then Haloperidol, then Risperidone, with the lowest rate for quetiapine (Newport et al., 2007a).

\subsubsection{Effects on ability to conceive}

All FGAs and some SGAs (especially risperidone and amisulpride) can cause hyperprolactinaemia and impaired fertility (Haddad and Wieck, 2004). Switching to a prolactin-sparing antipsychotic may lead to unwanted pregnancy. A woman planning a pregnancy and stopping a prolactin-raising antipsychotic may still have difficulties conceiving due to other factors, such as stress, obesity, and illness-related endocrine mechanisms. In men, hyperprolactinaemia can lead to loss of libido and reduction and impairment in spermatogenesis (De et al., 2003).

\subsubsection{Pregnancy outcomes}

There is a concern that antipsychotics, particularly SGAs associated with metabolic disturbance may increase the risk of gestational diabetes mellitus (GDM), obesity and gestational hypertension, conditions which are in turn associated with adverse maternal and neonatal outcomes such as fetal growth abnormalities, pre-term birth and congenital malformations. Studies comparing women on antipsychotics with healthy controls find a nearly two-fold increased risk of GDM (approximately 4\% vs 2\%) (Boden et al., 2012a; Reis and Kallen, 2008), but studies which employ a disease matched control group of psychiatrically ill unexposed women find no significant risk increase in GDM or gestational hypertension (Petersen et al., 2016; Vigod et al., 2015). The risk of GDM appears similar for olanzapine or clozapine versus other antipsychotics (Boden et al., 2012b; Vigod et al., 2015).

Some studies have reported that antipsychotics may be associated with an increased risk of small for gestational age babies (12\% vs $8 \%$ ), low birthweight babies (by 50-100g) and preterm births (on average 2 day shorter gestation) but few differences are found between medicated and unmedicated psychiatrically ill women 
(Boden et al., 2012a; Coughlin et al., 2015; Lin et al., 2010; Petersen et al., 2016; Terrana et al., 2015; Vigod et al., 2015). One systematic review reported similar risks for pre-term birth for SGAs and FGAs (Coughlin et al., 2015). A specific association with macrosomia (Boden et al., 2012b) and being large for gestational age (Gentile, 2010; Newham et al., 2008) has been suggested for SGAs, though other studies have not observed this (Boden et al., 2012c; Newport et al., 2007b).

There is no evidence for an increased risk of stillbirth or spontaneous miscarriage (Coughlin et al., 2015; Habermann et al., 2013; McKenna et al., 2005; National Institute for Health and Care Excellence, 2015a; Terrana et al., 2015; Vigod et al., 2015).

\subsubsection{Teratogenicity}

Existing evidence suggests that FGAs and SGAs are not major teratogens and, for those for which human data is available, few specific associations have been identified (Ennis and Damkier, 2015; National Institute for Health and Care Excellence, 2015a; Terrana et al., 2015). However, there are limited data on individual antipsychotics, with most first trimester exposure data available for olanzapine (1500 exposures) and quetiapine (440 exposures) (Ennis and Damkier, 2015; Vigod et al., 2015), and more recently for risperidone and aripiprazole (Huybrechts et al., 2016).

Systematic reviews estimate a $60 \%$ to two-fold increased risk for major congenital malformations among antipsychotic-exposed infants (Coughlin et al., 2015; Terrana et al., 2015), with an estimated absolute risk increase of 13/1000 from a background rate of 20-40/1000 (National Institute for Health and Care Excellence, 2015a). One systematic review reported similar risks for major congenital malformations following exposure to FGAs and SGAs (National Institute for Health and Care Excellence, 2015a). The commonest reported association is with cardiac defects (Coughlin et al., 2015), but such associations, like with cardiac septal defects, may be due to detection bias (Habermann et al., 2013; Reis and Kallen, 2008).

A recent national US cohort study of around 1.36 million women with a livebirth delivery in 2000-2010 found that the risk of any congenital malformations, and specifically of cardiac malformations, was not meaningfully increased for women exposed to atypical antipsychotics $(\mathrm{N}=9258)$ or typical antipsychotics $(\mathrm{N}=733)$ when compared to unexposed women after adjusting for key confounders (Huybrechts et al., 
2016). For individual antipsychotics, the authors found no increased risk following exposure to quetiapine $(\mathrm{N}=4221)$, olanzapine $(\mathrm{N}=1394)$, aripirazole $(\mathrm{N}=1756)$ or ziprasidone $(\mathrm{N}=697)$. They did find an unexpected small increased risk following risperidone exposure $(\mathrm{N}=1566)$ of any malformation by around $25 \%$ from around $3 \%$ in unexposed infants to $5 \%$ in those exposed and for cardiac malformations from $1 \%$ to $2 \%$. The authors argue that these findings for risperidone indicate a possible safety signal that requires clarification in future studies (Huybrechts et al., 2016). Other studies that have used psychiatric controls, or that controlled for confounding factors, have found no significant increase in congenital malformations (Boden et al., 2012a; Cohen et al., 2016; Petersen et al., 2016; Vigod et al., 2015), though risk estimates were imprecise. Earlier studies reported no significant increase in risk for major malformations with butyrophenones (mainly haloperidol) (Diav-Citrin et al., 2005) and phenothiazines (Slone et al., 1977).

\subsubsection{Neonatal and developmental problems}

Extrapyramidal and withdrawal symptoms have been described in neonates following late pregnancy exposure to antipsychotic medication (Gentile, 2010; Habermann et al., 2013; Sadowski et al., 2013). The US Food and Drug Administration (http://www.fda.gov/Drugs/DrugSafety/ucm243903.htm) and the UK's Medicines and Health Care Products Regulatory Authority (https://www.gov.uk/drug-safety-update/antipsychotics-risk-of-extrapyramidaleffects-or-withdrawal-symptoms-in-newborns) have issued relevant safety labelling, and recommended relevant monitoring for neonates. However, studies that adequately controlled for confounding did not find a specific association between antipsychotic exposure and poor neonatal adaptation (Petersen et al., 2016; Vigod et al., 2015).

There is very little evidence on the long-term neurodevelopmental outcome of infants exposed to antipsychotics in pregnancy. One small study found neuro-motor delay at 6 months in infants exposed to antipsychotics compared to infants exposed to antidepressants or unexposed infants (Johnson et al., 2012). Another study found development delay at 2 months but not at 12 months among infants of mothers with schizophrenia treated with antipsychotics (SGAs or sulpiride) compared to infants of healthy, unexposed mothers (Peng et al., 2013). 


\subsubsection{Breastfeeding}

Breastfeeding whilst on clozapine is contraindicated, due to the risk of infant agranulocytosis and seizures (National Institute for Health and Care Excellence, 2015a; Parikh et al., 2014; Uguz, 2016). Many FGAs (haloperidol, perphenazine, trifluoperazine and chlorpromazine) are excreted in breast milk in small amounts, with an estimated RID of less than 10\% (Moretti, 2009; Parikh et al., 2014). A 2016 review of the safety of SGAs in breastfed infants identified 37 studies with 210 exposed infants (including 170 exposed to olanzapine) (Uguz, 2016). The evidence suggested low RID values for olanzapine and quetiapine, moderate RID values for risperidone and aripiprazole and high RID values for amisulpride. No serious adverse effects were reported. There were case reports of drowsiness, irritability, motor abnormalities and poor feeding following exposure to these drugs and infants should be monitored appropriately especially if at high risk (e.g. premature or LBW infants).

There were a few case reports of neurodevelopmental delay in infants exposed to antipsychotics in breastmilk (Gentile, 2008; Parikh et al., 2014) but these were not controlled studies and the effect of the underlying illness was not taken into account. There are a lack of data on long-term neurodevelopmental outcomes.

\section{Conclusions/Recommendations}

- There is now more published safety data in pregnancy for SGA than FGA antipsychotics.

- For SGAs there is most reproductive safety data for quetiapine, olanzapine and risperidone, with more limited data for clozapine, aripirazole and ziprasidone.

- For FGAs there is most safety data for haloperidol.

- Quetiapine has a relative low rate of placental passage.

- This should be taken into account alongside the other factors influencing choice of medication in pregnancy discussed in section 4.

- Once confounders are taken into account there is little evidence of significant risk to maternal and infant outcomes for FGAs and SGAs. There is a possible safety signal for a small increased risk of malformations following in utero exposure to risperidone. Not all outcomes have been adequately studied and an increased risk cannot be completely ruled out.

- Prolactin elevating antipsychotics impair the fertility of women and men. 
- The risk of GDM may be increased by antipsychotics and enhanced diabetes screening for all women prescribed antipsychotics (with oral glucose tolerance test as the gold standard) is advised.

- SGAs may be associated with increased birthweights of babies.

- There are suggestions that antipsychotic exposure in utero may be associated with neuro-motor developmental delays but the limited nature of the data makes drawing conclusions in this regard difficult and longer term data is lacking.

- Switching medication is generally not advised due to the risk of relapse. Consider using the antipsychotic that has worked best for the woman after discussion of benefits and risks.

- Monitor any baby closely who was exposed near delivery or is breastfed by a mother taking antipsychotics.

- Breastfeeding whilst on clozapine is contraindicated.

- Recommendations regarding the use of antipsychotics in schizophrenia and bipolar disorder is describe in sections 6.4 and 6.3 respectively.

\section{$5.4 \quad$ Lithium}

Despite the frequency of its use in psychiatric practice over many decades, the body of evidence on reproductive safety is smaller for lithium than for many other established drugs prescribed for mood disorders.

\subsubsection{Effects on ability to conceive}

Lithium is not known to have significant effects on female fertility but may inhibit sperm motility (Raoof et al., 1989).

\subsubsection{Pregnancy outcomes}

The available data are too few to draw any conclusions regarding effects on spontaneous abortion rates, fetal growth in utero or risk of preterm delivery.

\subsubsection{Teratogenicity}

Two meta-analyses and one case control study have provided conflicting conclusions as to whether there is a small increase in the overall rate of major congenital malformations after first trimester lithium exposure (Diav-Citrin et al., 2014; McKnight et al., 2012; National Institute for Health and Care Excellence, 2015a). Much attention has focussed on the rare (prevalence in the general population 
1:20,000) but severe cardiovascular anomaly of the Ebstein type because the uncontrolled lithium baby register reported an increase of its prevalence to $2.7 \%$ (Weinstein, 1980). The methodological flaws of the register and the low number of Ebstein cases in subsequent studies suggest that, if there is any effect, it is likely to be much smaller, and that any effect estimates have to be regarded as unreliable (DiavCitrin et al., 2014; McKnight et al., 2012; National Institute for Health and Care Excellence, 2015a). Indeed the most recent and largest surveillance study of 5.6 million births identifying 173 cases of Ebstein's anomaly, unexplained by other causes, found an association with maternal mental health problems but not with lithium exposure (Boyle et al., 2016).

\subsubsection{Neonatal effects}

There are no systematic data in this area.

\subsubsection{Neurodevelopmental effects}

Small studies find no gross mental anomalies in children exposed to lithium in pregnancy compared to their unexposed siblings at age six (Schou, 1976).

\subsubsection{Breastfeeding}

The RID estimates of lithium vary widely with values of up to $42 \%$ being reported (Drugs and Lactation Database, 2016: https://www.nlm.nih.gov/pubs/factsheets/lactmedfs.html). Infant serum levels can reach up to $50 \%$ of the maternal value and cases of lithium intoxication and other adverse effects have been reported (Drugs and Lactation Database, 2016), though there are case reports of successful breastfeeding by women taking lithium with careful monitoring of infant lithium levels (Bogen et al., 2012; Viguera et al., 2007a).

Conclusions/Recommendations

- $\quad$ There are no known effects of lithium on fertility in females.

- Too little is known about the effects of lithium on pregnancy outcomes of spontaneous abortion, birth weight and gestational length to draw any conclusions.

- Due to the small volume of data, existing evidence cannot exclude an effect of lithium on overall malformation rate, or cardiovascular anomalies in particular. However, if a risk exists, it is likely to be small.

- There are no systematic data regarding neonatal effects of lithium exposure.

- There are limited data suggesting no effects of lithium on neurodevelopment. 
- Significant amounts of lithium pass into breast milk.

- Recommendations regarding the use of lithium in bipolar disorder are made in section 6.3.

\subsection{Antiepileptic Mood Stabilisers}

Evidence relating to the reproductive safety of antiepileptic drugs (AEDs) almost exclusively comes from studies of women with epilepsy.

\subsubsection{Endocrine and Effects on ability to conceive}

Women with epilepsy using valproate are more likely to have an increased rate of polycystic ovary syndrome/ovaries (Hu et al., 2011; Svalheim et al., 2015). However, evidence that this applies to women with bipolar disorder is lacking (Reynolds-May et al., 2014). Valproate treatment is reported to be associated with adverse spermatogenesis in men (Hamed et al., 2015).

Carbamazepine and lamotrigine do not appear to cause reproductive endocrine disorders (Svalheim et al., 2015). However, by reducing the efficacy of oral and parenteral contraceptives, carbamazepine increases the risk of unintended pregnancies.

\subsubsection{Pregnancy outcomes}

There are limited data in this area. It is important to note that a diagnosis of epilepsy is associated with a range of adverse obstetric outcomes, unrelated to AED use (Viale et al., 2015) and that the effect of different drugs may be very different. Current evidence suggests that the use of AEDs in pregnancy is not associated with still-birth (Bech et al., 2014; Viale et al., 2015), but in high doses there use is associated with a small increased rate of spontaneous abortions (16\% in women taking AEDs vs $13 \%$ in those not) (Bech et al., 2014). AEDs in pregnancy are also associated with a small (less than 50\% increase) but significant increased risk of some obstetric complications (post-partum haemorrhage, induction of labour, admission to neonatal intensive care, low birth weight) over and above that associated with epilepsy (Viale et al., 2015), though the data that this estimate is based on are not fully controlled for all potential confounders. Multiple AED drug treatment appears to increase risks (Viale et al., 2015).

\subsubsection{Teratogenicity}

Early pregnancy exposure to valproate is associated with an approximately 3fold increase in the rate of major congenital malformations (from around 3\% to 8 - 
11\%) (Meador et al., 2008; National Institute for Health and Care Excellence, 2015a). Significant associations have been reported for spina bifida (an approximate 13-fold increased risk from approximately $0.09 \%$ in the general population (Khoshnood et al., 2015) to between 1 and 2\%), atrial septal defects, cleft palate, hypospadias, polydactyly and craniosynostosis (Jentink et al., 2010b). Although teratogenicity may depend on daily valproate dosage, the reported critical thresholds vary widely (Tomson and Battino, 2012).

Early pregnancy exposure to carbamazepine has been reported to be linked with an approximately 2-fold increased rate of major congenital malformation (from around $2 \%$ to $5 \%$ ) in a meta-analysis (National Institute for Health and Care Excellence, 2015a) and an Australian register analysis (Vajda et al., 2016), although some studies have failed to show this association (Ban et al., 2015). Among anomalies, spina bifida has been found to be specifically more common, but the risk is smaller than in the case of valproate (an approximate 3-fold increased risk) (Jentink et al., 2010a).

A meta-analysis and current controlled studies of the teratogenic potential of lamotrigine (Ban et al., 2015; Dolk et al., 2016; National Institute for Health and Care Excellence, 2015a; Vajda et al., 2016) suggest that it is not associated with major congenital abnormalities, though one population study did find a small significant increase (from around 3\% to 5\%) (Ban et al., 2015).

\subsubsection{Neonatal effects}

Valproate use in the $2^{\text {nd }}$ or $3^{\text {rd }}$ trimester may be linked with increased neonatal problems (Kallen et al., 2013; National Institute for Health and Care Excellence, 2015a; Pennell et al., 2012) but the evidence base is limited.

\subsubsection{Neurodevelopmental effects}

Prenatal exposure to valproate is associated with lower IQ in school age children. The estimated difference to children of untreated women with epilepsy is approximately 8 IQ points (Bromley et al., 2014; National Institute for Health and Care Excellence, 2015a). In women with epilepsy, the risk of ASD is reported to be doubled from around $2 \%$ to $4 \%$ with in utero exposure to valproate, and childhood autism 3 fold from $1 \%$ to $3 \%$ (Christensen et al., 2013). These harmful effects of valproate may occur throughout fetal development. 
Current evidence does not suggest that carbamazepine is associated with poorer cognitive development (Bromley et al., 2014) or ASD (Bromley et al., 2014; Christensen et al., 2013; National Institute for Health and Care Excellence, 2015a). Although less is known about lamotrigine, current data also suggest that children are not affected in their neuro-development (Bromley et al., 2014; Christensen et al., 2013; McCorry and Bromley, 2015; National Institute for Health and Care Excellence, 2015a).

\subsubsection{Breastfeeding}

The serum levels of infants exposed to lamotrigine via breastmilk tend to be high, averaging 30-35\% of maternal serum levels (Drugs and Lactation Database, 2016: https://www.nlm.nih.gov/pubs/factsheets/lactmedfs.html). One case of severe apnoea and other cases of CNS depression have been reported (Drugs and Lactation Database, 2016). Concern has also been raised regarding the theoretical risk of dermatological problems in babies breast fed by mothers taking lamotrigine (Drugs and Lactation Database, 2016).

There are relatively low levels of valproate in breast milk (Drugs and Lactation Database, 2016). Carbamazepine levels are intermediate between valproate and lamotrigine (Drugs and Lactation Database, 2016) leading to a higher risk of infants experiencing side effects (sedation, poor sucking, withdrawal reactions and hepatic dysfunction) from ingestion.

\section{$\underline{\text { Conclusions/Recommendations }}$}

- Most data relating to the reproductive safety of anti-epileptic drugs come from women with epilepsy and their infants. There is no reason to believe that there are differences for the medications when taken for bipolar disorder.

- Valproate is associated with polycystic ovary syndrome and hence reduced fertility in women. It is also associated with impaired spermatogenesis.

- Carbamazepine can reduce the effectiveness of oral and parenteral contraceptives.

- Individual AEDs have variable and uncertain effects on pregnancy outcomes and data for AEDs studied as a group should not be used to counsel women regarding fetal risk.

- Valproate is associated with a significant increased risk of a range of major congenital malformations, particularly neural tube defects. 
- There is some data to suggest that carbamazepine increases the risk of major congenital malformations but a clear estimate of risk remains undefined.

- Lamotrigine is probably not associated with significant risks of structural teratogenicity.

- Supplementary folate does not appear to decrease the risk of neural tube defects and other major congenital malformations attributed to in utero exposure to valproate or other AEDs but is advised to protect against the occurrence of neural tube defects associated with low folate (but see recommendations for Lamotrigine, below).

- Valproate exposure in utero throughout pregnancy is associated with a decreased IQ in school age children and an increased risk of ASD.

- There is no evidence that carbamazepine or lamotrigine are associated with neurodevelopmental problems.

- Lamotrigine tends to pass in high concentrations into breast milk and caution is advised in using this in women who are breastfeeding. Carbamazepine is also found in breast milk at significant levels but valproate less so.

- It is recommended that valproate is not used in female children, adolescents or women of child bearing potential. This, together with the use of AEDs in bipolar disorder, is described in more detail in section 6.3.

\subsection{Drugs used in the treatment of ADHD}

NICE recommends the use of medication as first-line treatment in adults with ADHD and moderate or severe impairment (National Institute for Health and Care Excellence, 2008). Medications used to treat ADHD are increasingly being prescribed during pregnancy. A Danish register study found the rate increasing 5 to 533 per 100,000 person years between 2003 and 2010 (Haervig et al., 2014). It is therefore of concern that there is a paucity of data regarding their safety in pregnancy.

Prior to 2014 there were extremely limited data regarding the use of methylphenidate in pregnancy. A systematic review reported just 3 papers describing a total of just 41 children exposed (Bolea-Alamanac et al., 2014). Since then two Danish studies have reported a lack of observed congenital malformations following in utero exposure, including in the first trimester (Diav-Citrin et al., 2016; Pottegard et 
al., 2014). However it is important to note that the numbers of exposed cases reported is low and hence these observations can only exclude malformations occurring at a high frequency.

There have been suggestions that methylphenidate taken during pregnancy may increase the risk of miscarriage (Haervig et al., 2014). However there are data which suggest that this finding may be confounded by indication due to an increased risk with ADHD itself (Bro et al., 2015). Growth restriction and preterm delivery has been reported following abuse of methylphenidate (Debooy et al., 1993).

Data have suggested that methylphenidate or atomoxetine use is associated with an increased risk of low Apgar score at delivery, and that this is not seen in babies born to women with ADHD not on treatment (Bro et al., 2015). Neonatal withdrawal symptoms are to be expected following methylphenidate exposure in utero close to birth, as seen with other stimulants (see section 3.9.5).

There is little or no human data examining the effects of in-utero exposure to methylphenidate on neurodevelopment. Similar there is little data on the effect of breast feeding by mothers taking it.

There are even less data available regarding the safety of atomoxetine use in pregnancy and during breast feeding. Pharmacologically the drug is serotonin and noradrenaline reuptake inhibitor (SNRI) and may therefore have similar effects to those reported for similar drugs, such as venlafaxine, and SSRIs. It is therefore important to consider the possibility of adverse effects such as PPHN, though to date there have been no reports of this being linked to atomoxetine.

\section{$\underline{\text { Conclusions/Recommendations }}$}

- There are limited data regarding the risks of in utero exposure to methylphenidate and very little regarding atomoxetine.

- Current data do not suggest a link between methylphenidate exposure and congenital malformations.

- Methylphenidate may be associated with an increased risk of miscarriage, but this may be a result of confounds related to the diagnosis of ADHD.

- Methylphenidate exposure in utero close to delivery may be associated with a neonatal syndrome with low Apgar scores and stimulant withdrawal syndrome. 
- Risks associated with atomoxetine are undertermined. It is not known if it is appropriate to extrapolate from the data related to SNRIs and SSRIs.

\subsection{Drugs used in treatment of substance disorders}

Pharmacotherapy for substance misuse and dependence has been reviewed in other BAP guidelines (Lingford-Hughes et al., 2012), with more specific guidance on the integrated management of substance misuse in pregnant women in the NICE 'Pregnancy and Complex Social Factors guideline (2010) (National Institute for Health and Care Excellence, 2010a) and WHO guidelines (World Health Organisation, 2014). Other sources of information include: Confidential Enquiries into Maternal Deaths (Cantwell et al., 2011), Cochrane reviews on improving pregnancy outcomes (Whitworth and Dowswell, 2009) and reviews of psychosocial interventions and opioid maintenance programmes in pregnant women (Minozzi et al., 2013b; Terplan et al., 2015a).

\subsubsection{Smoking/nicotine dependence}

A recent Cochrane review of six trials of nicotine replacement therapy (NRT) enrolling more than 1700 pregnant smokers found no differences between NRT and control groups in rates of miscarriage, stillbirth, premature birth, birthweight, low birthweight, admission to neonatal intensive care, caesarean section, congenital abnormalities or neonatal death. They concluded that further research evidence on NRT efficacy and safety is needed, ideally from placebo-controlled RCTs (Coleman et al., 2015). NICE recommend that NRT should be considered after review of all the risks and benefits if interventions without NRT have failed (National Institute for Health and Clinical Excellence, 2010d).

Bupropion is licensed in the UK for smoking cessation but in many other countries it is licensed as an antidepressant. Most of the limited data on the drug in pregnancy come from its use to treat depression and do not suggest adverse pregnancy outcomes (Chun-Fai-Chan et al., 2005). There have been reports of cardiac defects in infants exposed in utero (Alwan et al., 2010; Louik et al., 2014) but the studies were small, did not fully account for all potential confounders and the nature of the cardiac abnormalities was inconsistent between the studies. Additional studies have also not replicated the findings (Huybrechts et al., 2014b; Thyagarajan et al., 2012). There are very limited data relating to neonatal or neurodevelopmental effects of bupropion 
exposure in utero. As a result of the limited data available, NICE has recommended that bupropion should not be used in pregnancy (National Institute for Health and Care Excellence, 2010b), though there are reports of its successful use (Berard et al., 2016; Nanovskaya et al., 2016).

A recent international prospective cohort study showed no increase in major malformation rate amongst infants of women who used varenicline during pregnancy (Richardson et al., 2016). However, the sample size was small, elective termination rate amongst this cohort was high, and in most cases use was discontinued prior to the major risk period for structural teratogenesis. As there are currently no other published epidemiological studies, fetal risk is undetermined and use in pregnancy is not advised.

\subsubsection{Alcohol misuse/dependence}

The main options for alcohol detoxification in pregnancy are chlordiazepoxide and diazepam (Lingford-Hughes et al., 2012): see section 5.2 on adverse effects of anxiolytics, though there needs to be caution extrapolating from data regarding prolonged use of anxiolytics for an anxiety disorder and shorter term, but often higher dose, for use during detoxification. Despite chlormethiazole being used in the past as a treatment for eclampsia there are no epidemiological data of its use in pregnancy from which to assess its safety. Anticonvulsants (see section 5.5) should only be used if the benefits outweigh the risks of fetal malformation or other adverse effects (Lingford-Hughes et al., 2012).

There is an absence of evidence and clinical data on the use of relapse prevention medications (acamprosate, naltrexone and nalmefene) for alcohol dependence in pregnant or breastfeeding women (DeVido et al., 2015). There are limited data regarding disulfiram use in pregnancy though the severe autonomic instability that can occur with co-ingestion of alcohol would suggest a theoretical risk with use in pregnant women (DeVido et al., 2015).

\subsubsection{Opioid misuse/dependence}

Methadone and buprenorphine treatment in pregnancy is based on a considerable body of evidence on management of illicit opioid use in the general population (National Institute for Health and Care Excellence, 2007) and this treatment has been found to improve maternal and fetal outcomes compared to those seen with untreated opioid misuse (Zedler et al., 2016). Methadone in pregnancy is 
associated with improved antenatal care, reduced maternal morbidity and improved neonatal outcomes (Burns et al., 2007) although a recent study found low birth weight in those on treatment compared to general population (Mactier et al., 2014). There are less data regarding buprenorphine in pregnancy, though evidence is increasing (Jones et al., 2010; Minozzi et al., 2013b; Wurst et al., 2016). In a recent meta-analysis comparing pregnancy outcomes, buprenorphine was associated with around half the risk of preterm birth and with greater birth weight (around 10g) compared with methadone (Zedler et al., 2016). No treatment differences were observed for spontaneous fetal death, fetal/congenital anomalies and other fetal growth measures, although the small sample sizes limits the power to detect such differences (Zedler et al., 2016).

A Cochrane review found no difference between methadone and buprenorphine in rates of neonatal abstinence syndrome (NAS) needing treatment (Minozzi et al., 2013a). The single large RCT in this review - the MOTHER study did find babies of mothers treated with buprenorphine had shorter treatment for NAS, required less medication for NAS and had shorter stays in hospital (Jones et al., 2010).

\subsubsection{Stimulant misuse/dependence}

There is no evidence for any substitute or relapse prevention pharmacological treatments. Psychosocial treatments with some form of contingency management have the best evidence base in the general population although there is little evidence for the effectiveness of psychosocial interventions in pregnant drug using women (Knapp et al., 2007; Terplan et al., 2015b).

\section{$\underline{\text { Conclusions/Recommendations }}$}

- The current available data on NRT suggest that it is safe to use in pregnancy.

- Data regarding bupropion and varenicline use during pregnancy are extremely limited.

- Safety of benzodiazepines, which may be used in alcohol detoxification is described in section 5.2

- There is a lack of human data on the safety of acamprosate, naltrexone and nalmefene. There is a theoretical risk of disulfiram in pregnancy if coingested with alcohol. 
- Methadone and buprenorphine use in women with opioid dependency is associated with improved fetal and maternal outcomes.

- There are no recommended pharmacological interventions for stimulant, cannabis or novel psychoactive substance use in pregnancy.

- Recommendations regarding the use of pharmacological interventions for substance misuse in the perinatal period are described in section 6.7.

\subsection{Neuromodulatory treatments}

Given that it is impossible to fully exclude all risks associated with psychotropic medication use in pregnancy, it is not surprising that women with depression, for example, find psychotherapy more acceptable than pharmacotherapy (Goodman, 2009). However another alternative option to pharmacotherapy is neuromodulatory treatment. A range of neuromodulatory treatments exist in psychiatry, the oldest being ECT and the use of this in pregnancy has recently been reviewed (Kim et al., 2015).

\subsubsection{ECT in the perinatal period}

ECT is not contraindicated in pregnancy: however given that an anaesthetic and muscle relaxant are required, it is not 'medication-free'. There have been no RCTs assessing the safety of ECT in pregnant or breastfeeding mothers. A retrospective review of cases between 1941 and 2007 (Anderson and Reti, 2009) reported on 339 cases, though the quality of the data was not very robust. There was one fetal death secondary to status epilepticus. Overall the rate of fetal and maternal adverse outcomes possibly related to the ECT was around $10 \%$ but most were transient and not life threatening to mother or fetus. These included fetal arrhythmias, vaginal bleeding and uterine contractions. There was one miscarriage in the first trimester 24 hours post ECT and one case of multiple cortical and deep white matter infarctions after multiple ECT courses but causality linked to ECT is hard to establish. A more recent systematic review examined 67 published case reports and case series including 169 women treated with 1,187 sessions of ECT (Leiknes et al., 2015). Women had been treated for a variety of disorders including depression, bipolar disorder and schizophrenia. There were no maternal deaths but an overall child 
mortality rate of $7 \%$, vaginal bleeding in $12 \%$, premature labour in $28 \%$, fetal cardiac arrhythmias or bradycardia in $43 \%$ and fetal malformations in $20 \%$ of cases reported. However given that these data are based on selectively reported cases, with no control population, and very mixed standards of reporting in publications from 1942 to 2013, it is unclear what weight to place on these findings. Note that it is reported that the risk of fetal bradycardia may be lower with propofol rather than methohexital (De Asis et al., 2013).

There are little long term follow up data of babies born to mothers treated with ECT during pregnancy other than a small case series and case reports and further data is required (Kim et al., 2015).

Overall, ECT is considered a possible treatment for severe depression during pregnancy but it should be used with caution, especially if there is a risk of vaginal bleeding or preterm birth (Kim et al., 2015; National Institute for Health and Care Excellence, 2015a). Guidance exists for its use (Kim et al., 2015; Miller, 1994; O'Reardon et al., 2011; Royal College of Psychiatrists, 2012).

There are virtually no data on the use of ECT in breastfeeding mothers. ECT may have a more rapid effect in postpartum psychosis than in non-postpartum psychosis (Reed et al., 1999). There is no reason to suspect that ECT is not efficacious in conditions which are known to respond to ECT at other times of life. Breastfeeding can be resumed as soon as the mother has recovered sufficiently from anaesthesia and neuromuscular blockade. It is important to consider the risks of venous thrombosis in the postpartum mother (Miller, 1994; Royal College of Psychiatrists, 2012).

\subsection{2 rTMS during pregnancy}

Because of the lack of a need for anaesthetic or muscle relaxant during rTMS, this offers an option that is completely free of medication. In a survey of 1,500 pregnant women, psychotherapy was identified as the favoured treatment option for depression during pregnancy. However, after being provided with information about rTMS, this was identified as an acceptable treatment option by only 16\% (Kim et al., 2011b). There are limited data regarding the use of rTMS during pregnancy. An open study of 10 women reported 7 as responding, with no observed adverse pregnancy or fetal outcomes (Kim et al., 2011a). A subsequent open study in 30 women also reported no 
complications (Hizli et al., 2014). Additionally a follow up study compared 18 children born to mothers who received rTMS during pregnancy with 26 children born to mothers with untreated depression during pregnancy (Eryilmaz et al., 2015), finding no difference in cognitive or motor development at around 30 months of age.

\subsubsection{Other neuromodulatory treatments}

Like rTMS, transcranial direct current stimulation (tDCS) does not require any anaesthetic or muscle relaxant. As such it may be an option for women keen to avoid medication. However there are no data regarding its use in the perinatal period. Deep brain stimulation (DBS) and vagus nerve stimulation (VNS) require surgical implantations, usually under general anaesthesia. However, they may be implanted prior to conception. There is a case report of three women with healthy pregnancies who had had DBS implanted for dystonia (Paluzzi et al., 2006), but there are no data in women with mental illnesses where electrode placement within the brain is different. There is one case report of a woman with VNS for depression who had a healthy pregnancy (Husain et al., 2005) and a similar one of a woman who had VNS for intractable epilepsy (Houser et al., 2010).

\section{Conclusions/Recommendation}

- The level of risks with ECT in pregnancy are unclear. Consider using ECT with caution in pregnant women for treatment of conditions where ECT is known to be effective (severe depression, severe mixed affective states, severe mania or catatonia) and in whom their physical health, or that of the baby, is at serious risk, or where there is insufficient time to wait for improvement with psychotropic medication. Follow normal good ECT practice as well as specific guidance for the use of ECT during pregnancy.

- ECT may be a useful option in breastfeeding women where there may be significant risks of medication to the nursing infant and/or the mother's ability to care for her child.

- There are very limited data on rTMS during pregnancy or breastfeeding, but this may offer a medication free treatment option for some women.

- $\quad$ There are no data for the use of tDCS in the perinatal period and very limited data regarding DBS and VNS. 


\section{RECOMMENDATIONS FOR THE PHARMACOLOGICAL MANAGEMENT OF SPECIFIC DISORDERS}

This section should be read in conjunction with section 4 that describes the general principles of prescribing in the perinatal period, particularly sub section 4.2. The advice in section 4 is relevant to all psychiatric disorders. A key point worth repeating is that it is always worth considering non-pharmacological management options, such as psychological interventions, as possible alternatives to medication. This point will not be reiterated in each of the sections below which will focus on pharmacotherapy.

\subsection{Management of depression}

\subsubsection{Pre-conception}

- Decisions on whether to continue or commence antidepressant treatment need to be individualised. See section 4.2 regarding factors influencing decisions and section 5.1 for specific risks of antidepressants in the perinatal period.

- None of the currently available antidepressants is absolutely contraindicated in pregnancy. Most guidelines recommend SSRIs as first line pharmacological treatments for depression (Cleare et al., 2015; National Institute for Health and Care Excellence, 2009). Additionally, the largest evidence base around reproductive safety is for the SSRIs. As a result this makes them first choice treatments preconception and in pregnancy for antidepressant naïve women.

- For non-antidepressant naïve women decisions about which antidepressant to use should follow the generic guidance around choice of drug laid out in section 4.2 .

- Care should be taken in women with treatment resistant depression who are being prescribed medication to augment their antidepressants. In particular it should be noted that there is no evidence to support the use of anticonvulsant mood stabilisers as adjunctive agents for unipolar depression (Cleare et al., 2015; Haddad et al., 2015). Given the particularly high risks associated with 
valproate, use in women of childbearing potential with unipolar depression should be avoided altogether.

\subsubsection{Antenatal}

- Note all of the recommendations for the pre-conception period (6.1.1) above.

- In general, antidepressants should not be discontinued suddenly on discovering a pregnancy due to risks of discontinuation syndrome and relapse.

- In late pregnancy, there is a risk/benefit decision to be considered around the risk of PPHN that is associated with antidepressants.

- There is a lack of evidence and consensus on whether reduction/discontinuation or maintenance of antidepressant close to delivery will alter the risk of poor neonatal adaptation. Discontinuing antidepressants before delivery may place the mother at an increased risk of relapse and importantly postnatal depression.

- $\quad$ There are very limited data on rTMS during pregnancy, but this may offer a medication free treatment option for some women.

\subsubsection{Postnatal}

- There is substantial evidence that women who are depressed in pregnancy are at an increased risk of depression postnatally. For these women, if antidepressant treatment is required in pregnancy, it is likely to be of benefit for it to continue in the postpartum period.

- Women who are well in pregnancy, but who have had a previous episode of depression postnatally, may also be at increased risk of subsequent postnatal recurrence. However there is limited evidence for pharmacological interventions that can reduce this risk and decisions should be made on an individual basis.

- Pharmacological treatment of depression postnatally does not differ substantially from depression at other times. The most distinctive consideration is in relation to breastfeeding (see section 4.8).

- As in choosing medication in pregnancy, there is a different risk/benefit analysis to be made depending on whether a woman is initiating antidepressant treatment or is already taking medication. For initiation, sertraline is associated with low transfer into breastfed 
babies and associated adverse effects. However the risks of other antidepressants may be outweighed by their clinical advantages for continuation treatment.

- It makes good clinical sense that choice of antidepressant in pregnancy takes into account breastfeeding intentions, avoiding the need to consider alteration of treatment in the early postpartum period, at a time of heightened risk.

\subsection{Management of anxiety disorders and insomnia}

- The impact of untreated anxiety and insomnia during pregnancy is significant (see section 3.4). There is uncertainty as to whether treatment decreases any long term risks to the child but this should not be a reason not to treat if maternal symptoms are affectinfg maternal wellbeing.

- Decisions on whether to continue or commence treatment need to be individualised. See section 4.2 regarding factors influencing decisions including between medication and non-pharmacological options.

- If the disorder is causing severe distress and/or impairment then medication may be required (Baldwin et al., 2014).

- In choosing a medication consider the woman's previous treatment history and, especially if treatment nä̈ve, recommendations regarding treatment in non-pregnant patients (Baldwin et al., 2014).

- SSRIs are first line pharmacological agents for generalised anxiety disorder, panic disorder, social anxiety disorder, PTSD and OCD in non-pregnant patients (Baldwin et al., 2014). Given the relatively large data base regarding their safety in the perinatal period, they are also the first line treatments for these anxiety disorders pre-conception and antenatally (see sections 5.1 and 6.1). Be aware of the limited data regarding other anxiolytics in pregnancy (see section 5.2).

- It is important to note that insomnia postnatally (inability to sleep even when not being disturbed by the baby) may herald or even precipitate severe illness such as postpartum psychosis (see below).

- Review the need for treatment regularly and use for the shortest period necessary. 


\subsection{Management of bipolar disorder}

\subsubsection{General management issues across all childbearing stages}

- The aim of management is to achieve optimal mood stability at a critical time in the woman's life.

- Because of the complexities and risks of bipolar disorder in the perinatal period, treatment decisions need to be based on an up-to date and in depth psychiatric assessment of the woman at the first psychiatric contact in the preconception or perinatal period (see section 4.2).

- Raise the woman's awareness that stressors, irregular activity patterns and sleep disturbance can trigger bipolar relapse (Goodwin et al., 2016). Minimizing them is particularly challenging at the end of pregnancy and in the postnatal period. The mental health team should ensure that the woman has appropriate support and strategies in place to reduce their impact.

- Given the high rate of substance misuse (including alcohol and tobacco) seen in bipolar disorder, it is important to discuss measures to reduce this.

- Self-monitoring by, for example, using mood diaries is an important element of the management of bipolar disorder in any patient at any time (Goodwin et al., 2016).

- Since there is no or insufficient trial data for pregnant or postnatal patients with bipolar disorder, the efficacy of different medication options needs to be extrapolated from other patient groups, and from the experience of the individual patient.

\subsubsection{Pre-conception and antenatally}

- Because antipsychotics have a better risk/benefit ratio than valproate and carbamazepine, and a larger reproductive evidence base and possibly better reproductive safety profile than lamotrigine and lithium, they generally form the mainstay of the treatment of women in the perinatal period (see sections 5.3, 5.4 and 5.5). SGAs generally have a larger evidence base than FGAs for use in bipolar disorder (Goodwin et al., 2016).

- If a woman planning a pregnancy is taking an antipsychotic drug with prolactin elevating potential, she should be asked about her menstrual cycle. Any patient reporting oligo- or amenorrhoea should have a 
prolactin level measured. If she has hyperprolactinaemia that is thought to be caused by antipsychotic medication, consider switching medication to a prolactin-sparing agent, such as quetiapine or olanzapine (Haddad and Wieck, 2004).

- During pregnancy, prolactin raising antipsychotics can be prescribed.

- In general valproate should not be prescribed to any female child or adolescent, or woman who is of childbearing potential, pregnant or planning a pregnancy (National Institute for Health and Care Excellence, 2015a).

- Note that the Medicines and Health Products Regulatory Authority in the UK (2015) considers an exception to this advice for a group of women with childbearing potential who have not responded to any other medication (https://www.gov.uk/drug-safety-update/medicines-related-tovalproate-risk-of-abnormal-pregnancy-outcomes). These women can be prescribed valproate if they have mental capacity, if pregnancy is excluded and they are well enough to make consistent use of highly reliable contraception. A toolkit for clinicians and patients is available (https://www.gov.uk/government/publications/toolkit-on-the-risks-ofvalproate-medicines-in-female-patients).

- If a woman planning a pregnancy is already taking valproate, it should (unless exceptional circumstances pertain) be withdrawn gradually over at least 4 weeks (Goodwin et al., 2016) whilst the woman uses effective contraception.

- Should a woman become pregnant whilst taking valproate it should usually be withdrawn. Consider replacing it with an antipsychotic drug.

- In general, do not initiate carbamazepine in preconception or pregnant women because of its teratogenic potential and uncertain efficacy in bipolar disorder (Goodwin et al., 2016).

- If a woman is already taking carbamazepine, discuss with the woman the possibility of stopping the agent.

- If lamotrigine is used in pregnancy be aware that:

- Serum concentrations of total and free lamotrigine decline markedly due to pregnancy-induced alterations in the activity of glucuronosyl transferases and renal function. The extent is highly variable (Tomson et 
al., 2013). To aid the management of depressive relapses in the perinatal period, serum levels should be monitored from preconception, if possible, until the first month postpartum when levels should return to prepregnancy values.

- Preliminary evidence suggests that folic acid supplements may reduce the effectiveness of lamotrigine (Geddes et al., 2016).

- There is little evidence that the use of folic acid mitigates AED induced harm to the fetus. NICE guidelines for epilepsy recommend high dose folic acid before any possibility of pregnancy for women on AEDs including lamotrigine (National Institute for Health and Care Excellence, 2012). However, there is uncertainty regarding the effectiveness of this (see sections 4.2 and 5.5.3). There is also the concern regarding the effect of folate on the efficacy of lamotrigine (see above). Nevertheless, it is recommended to prescribe a high dose (5mg/day) in the three months before and after conception, certainly for patients on valproate and carbamazepine and possibly those on lamotrigine.

- If lithium is prescribed during pregnancy:

- Inform the obstetrician of any first trimester exposure and request particular attention towards fetal screening for cardiovascular anomalies.

- If the plan is to discontinue lithium it should be tapered gradually over 4 weeks. Consider restarting in the second or third trimester.

- Lithium levels tend to decrease in pregnancy but can also sometimes increase again, particularly in late pregnancy. Measure plasma levels once a month until 36 weeks of pregnancy and then weekly until delivery. As ever, it is important that women are instructed to avoid dehydration.

- Women taking lithium should deliver in hospital and be monitored by the obstetric team.

- To prevent maternal and neonatal intoxication, lithium should be suspended for 24-48 hours before a planned Caesarean section or induction, and at the onset of labour (Newport et al., 2005) and the lithium level should be measured 12 hours after the last dose. If levels are not above the therapeutic range, restart lithium on day 1 postnatal and check the level again after one week. 
- Stopping medication other than lithium before delivery is not known to have any benefits for the neonate but may place the mother at a greatly increased risk of bipolar relapse.

\subsubsection{Postnatal}

- Postnatal bipolar recurrences and new onset bipolar and other affective postpartum psychoses tend to commence suddenly in the first few days after delivery, progress rapidly and be severe. They pose serious risks to the safety of mother and child. These illnesses need to be identified early, regarded as psychiatric emergencies and managed effectively with medication.

- In women who have pre-existing bipolar disorder and who are unmedicated in pregnancy, re-starting previously effective medication, or an antipsychotic such as quetiapine or olanzapine, on the first day after childbirth may prevent some, but not all, early recurrences.

- The duration of treatment that should be used for prophylaxis postnatally is uncertain. In the absence of such evidence it is recommended to follow recommendations around prophylaxis in the non-perinatal situation (Goodwin et al., 2016; National Institute for Health and Care Excellence, 2014).

- In severe postpartum affective states that are associated with high risks or poor response to medication, ECT should be considered (see section 5.8.1).

- Any antipsychotic medication that is effective in bipolar disorder can be considered during breastfeeding with the possible exception of lurasidone (due to a limited evidence base) and clozapine (due to risks of agranulocytosis - see section 5.3).

- Lithium, valproate and carbamazepine should not be prescribed to women with affective disorders who breastfeed.

- Lamotrigine can be prescribed during breastfeeding but infants should be carefully monitored for potential side-effects. If there is any concern, consider measuring infant serum levels. Discontinue lamotrigine if the baby develops a skin rash until the cause has been established (Drugs and Lactation

Database, 2016: https://www.nlm.nih.gov/pubs/factsheets/lactmedfs.html). 


\subsection{Management of schizophrenia and psychosis}

\subsubsection{Management across all childbearing stages}

- Take into account the general principles laid out in section 4.2.

\subsubsection{Pre-conception}

- Discuss contraception and pregnancy plans with all women of reproductive age who have schizophrenia or other psychosis. Do not assume impaired fertility. Note that if switching to a prolactin-sparing drug discuss fertility changes.

- If a woman who is on a prolactin-raising drug is struggling to get pregnant consider switching to a prolactin-sparing antipsychotic. She may still have difficulties conceiving due to other factors, such as stress and obesity.

- Discuss the risk of relapse in pregnancy (similar to risk of relapse outside the perinatal period) and in the postpartum period (higher than the risk of relapse outside the perinatal period, but not as high or as sudden as in bipolar disorder).

- If the woman is taking an antipsychotic which is effective for her, continue with this medication unless the evidence on risks outweigh the benefits.

- Consider that the risk of switching to an alternative antipsychotic is that beneficial effects on the mother's illness may be lost with the fetus still exposed to some risk.

- If, after taking these considerations into account, there is a decision to switch antipsychotic, choose the drug with the lowest risk profile for the woman, fetus and baby, taking into account a woman's previous response to medication.

- If she is established on clozapine, continue with this medication if the benefits are likely to outweigh the risks. Screen for diabetes and monitor fetal growth. Advise the woman that breastfeeding whilst on clozapine is not recommended (risk of infant agranulocytosis).

- If she is established on depot, continue with this medication if the benefits outweigh the risks, and in particular if there is a history of poor compliance with oral medication and a high risk of relapse. Ensure all health professionals are aware of drug pharmacokinetics and the need for prolonged monitoring for neonatal symptoms and any other suspected adverse effects. 


\subsubsection{Antenatal}

- If a woman decides to stop an antipsychotic, ensure she is offered optimal psychosocial interventions and monitored closely. If there is a deterioration in her mental state, consider the need for admission or the use of the compulsory detention.

- Discuss breastfeeding plans, bearing in mind the likely need to continue antipsychotics in the high-risk postnatal period.

\subsubsection{Postnatal period}

- The risk of relapse is higher in the postnatal period than during pregnancy, so maintain effective treatment.

- Avoid switching or stopping medication in the postnatal period unless there is good reason for doing so (e.g. serious adverse maternal effects such as sedation).

- Encourage women who are taking an antipsychotic to breastfeed, unless they are taking clozapine.

- However, monitor infants for sedation, feeding problems, motor abnormalities and neurodevelopment.

\subsection{Management of eating disorders}

\subsubsection{Pre-conception}

- A healthy BMI should be achieved prior to assisted reproduction (National Institute for Health and Care Excellence, 2013).

- Specialist ED treatment should be provided for women who have a history of an ED and who are experiencing difficulties conceiving. Ideally a reduction in symptoms should be achieved prior to conception.

- Unplanned pregnancies are common amongst women with AN, as women may assume that they are unable to conceive due to menstrual irregularities (Bulik et al., 2010; Easter et al., 2011; Micali et al., 2014). Routine pre-conception advice and psycho-education should therefore be provided.

\subsubsection{Antenatal}

- Women with ED often do not disclose their ED to healthcare professionals in the antenatal healthcare setting (Taborelli et al., 2015; Tierney et al., 2011). 
It is therefore recommended that questions relating to eating and weight control behaviours are routinely asked in early pregnancy, especially in high risk women (see e.g. (National Institute for Health and Care Excellence, 2004).

- Women who describe ED symptoms or have a threshold or sub-threshold ED in the perinatal period should be offered a multi-disciplinary assessment and specialist treatment where appropriate.

- Pharmacological treatment for ED has not been investigated in pregnant women.

- Given the lack of pharmacological treatments for AN, drug treatment might only be beneficial in treating comorbid physical or psychological conditions.

- Although fluoxetine is a treatment option for BN, CBT is the first line treatment and might be more appropriate during pregnancy.

- Similarly, although pharmacological options are available for BED, given the stronger evidence of efficacy for psychological treatments, these are recommended in pregnancy.

- Most women with current or past ED will be unsure about adequate nutritional requirements and will be intensely preoccupied with the adequate amount of weight gain needed and specific macro and micronutrient nutritional requirements. They might benefit from dietetic support and management.

- The US Institute of Medicine recommends specific weigh gain ranges depending on women's BMI (Rasmussen et al., 2009).

- Women who fail to gain adequate weight gain and continue to be underweight $(B M I<18.5)$ during pregnancy are at risk for adverse birth outcomes and might require intensive multi-disciplinary treatment, nutritional management and in severe cases hospitalization and statutory assessment.

\subsubsection{Post-natal}

- Given the high likelihood of ED relapse or recurrence post-partum (Bulik et al., 2007) and higher prevalence of post-natal depression (Easter et al., 2015; 
Micali et al., 2011b), women with active ED or history of ED should receive psychiatric monitoring postnatally.

- Studies show that mothers with ED have difficulties in feeding their infants (Micali et al., 2011a) and might find motherhood generally difficult. They are therefore likely to benefit from specific support during the first 6-months of life.

\subsection{Management of ADHD}

- There are limited data regarding the safety of medications used to manage $A D H D$ in the perinatal period (see section 5.6). There are more data with regards to methylphenidate exposure than for atomoxetine.

- The decision as to whether medication should be continued during pregnancy and breast feeding, and the choice of which to use should follow the principles laid out in section 4.

\subsection{Management of substance misuse}

- The WHO guidelines on the identification and management of substance use in pregnancy (World Health Organisation, 2014) are recommended.

- Women should be asked about the use of illicit drugs and supported to stop and remain abstinent.

- It is important to encourage early and accessible access to antenatal care, and provide integrated care with primary care, addiction services, obstetric and perinatal services, with emphasis on child safeguarding, good communication and planning for support for mother and baby (Knight et al., 2015). Harm reduction information should be provided.

\subsubsection{Smoking/nicotine dependence}

- All pregnant women who smoke should be referred to smoking cessation services and psychosocial interventions known to be effective should be offered.

- Nicotine replacement therapy is preferable to smoking and associated with better pregnancy outcomes.

- NRT should be offered after risk benefit analysis if other non-pharmacological interventions have failed. 
- Bupropion and varenicline should not generally be prescribed during pregnancy or breastfeeding.

\subsubsection{Alcohol misuse/dependence}

- NICE provides guidance on the detection and treatment of alcohol misuse in pregnancy (National Institute for Health and Care Excellence, 2010a; National Institute for Health and Care Excellence, 2011).

- The UK Chief Medical Office's advice (UK Chief Medical Officer, 2016) is unambiguous: "if you are pregnant or planning a pregnancy, the safest approach is not to drink alcohol at all, to keep risks to your baby to a minimum".

- Pregnant women should be screened for alcohol use with advice on cessation or at the least using minimal amounts. In general, advice on fetal alcohol syndrome should be readily available.

- Psychological and educational interventions may result in increased abstinence from alcohol, and a reduction in alcohol consumption among pregnant women (Stade et al., 2009), but there have been no trials in women with both mental disorders and alcohol abuse.

- Chlordiazepoxide or diazepam are the treatments of choice for alcohol detoxification in pregnant women requiring a medicated withdrawal of alcohol. They should ideally be used in an inpatient setting.

- Due to a lack of human data, acamprosate, naltrexone, nalmefene and disulfiram are not recommended for use in pregnancy or lactating women. (See section 5.7.2).

\subsubsection{Opioid misuse/dependence}

- Given evidence of the benefit of their use in pregnancy, methadone and buprenorphine treatment should be offered to those dependent on opioids as part of an integrated and comprehensive treatment programme including addiction services, primary care and obstetric staff.

- The choice of methadone or buprenorphine should be based on individual need and the woman's preference with informed consent after a comprehensive assessment. 
- The aim of treatment should be to eliminate illicit drug use for those commencing treatment or prevent relapse to illicit drug use in those already on substitution therapy.

- Pregnant women already receiving buprenorphine should not be advised to transfer to methadone unless they are not responding well to buprenorphine treatment (World Health Organisation, 2014).

- Buprenorphine-naloxone combination medication should not be used.

- Pregnant women already receiving methadone substitution treatment should be advised not to transfer to buprenorphine due to the risk of opioid withdrawal.

- The dose of methadone to be used in pregnancy is a matter of debate: enough to limit illicit drug use, or to use lowest dose to avoid a NAS (Gray et al., 2010).

- $\quad$ There is little evidence for detoxification.

- Slow reduction with monitoring can be undertaken during second and third trimester, provided stability is maintained in all aspects of care.

- Pregnant opioid dependent women should be encouraged to access opioid maintenance treatment rather than attempt detoxification, on the basis that relapse rates are high and the maternal and fetal risks are greater from failed detoxification and from relapse to illicit drug use than from opioid maintenance treatment (World Health Organisation, 2014).

- Management of pain in labour should be discussed with the woman and obstetric team.

\subsubsection{Stimulant misuse/dependence}

- Pregnant women are advised to stop all stimulants, including all novel psychoactive substances.

- Psychosocial interventions should be offered.

- There are no substitute pharmacological treatments that are recommended.

\subsubsection{Benzodiazepine misuse/dependence}

- Pregnant women misusing benzodiazepines should have a comprehensive assessment of their drug use. 
- Aim for gradual dose reduction with a long acting benzodiazepine e.g. diazepam (World Health Organisation, 2014).

- The lowest effective dose to prevent maternal withdrawal symptoms should be used.

- A slow reduction is recommended with close liaison with the obstetric team.

- Psychosocial interventions should be provided alongside pharmacotherapy and inpatient admission considered.

\subsection{Management of personality disorders}

- There is little or no data regarding the pharmacological management of personality disorders in the perinatal period.

- Follow the generic prescribing recommendations made in section 4. Given the paucity of data of efficacy of psychotropics in personality disorders, it is particularly important to consider carefully the risk/benefit balance of the medication.

- Often pharmacotherapy in personality disorders is used on a symptomatic basis. In this regard, follow the recommendations in the relevant sub-section of section 6.

\subsection{Management of acute behavioural disturbance}

- Acute behavioural disturbance may occur in pregnant and postnatal women in the context of severe mental illness, whether the condition occurs de novo or is a result of relapse of a previous or known condition. Timely and appropriate management is important because of the particular risks that the disturbance may present in the perinatal period i.e. physical risks to the pregnant woman, risks to the delivered woman who may be physically compromised in relation to recent childbirth (e.g. post caesarean section, sepsis etc.), physical risks associated with the environment (e.g. in theatre, labour ward, birthing pool etc.) and not least the risk a mother's disturbed behaviour may pose to a vulnerable infant. 
- Good practice in relation to prevention of behavioural disturbance and deescalation techniques must be followed.

- Be particularly aware of the increased risks of behavioural disturbance in the perinatal period due to:

- The risk of sudden and severe relapse postnatally in women with bipolar affective disorder

- The risk of a woman developing a psychotic episode for the first time post-childbirth

- The risk of relapse associated with non-adherence to medication where a woman does not take her medication due to fears that the fetus/ infant will be adversely affected.

- Clinicians in both psychiatric and obstetric units should be familiar with how to manage acute behavioural disturbance in the perinatal period.

- Women who are at a known risk of relapse and attendant behavioural disturbance should have a clear plan in their records as to how this will be managed (including what medication might be used) and this plan should be shared with all professionals and services who work with the woman.

- The aim of rapid tranquillization is to:

- Avoid prolonged physical intervention.

- Prevent / reduce harm to woman physically and psychologically.

- Prevent harm to others including the fetus/infant.

- A pregnant woman requiring rapid tranquillization should be treated according to the NICE clinical guidelines for the short-term management of Violence and aggression (National Institute for Health and Care Excellence, 2015c) as well as relevant disorder specific guidance.

- The mother should NEVER be secluded or left alone after rapid tranquillization.

- Restraint procedures should be adapted to avoid possible harm to the fetus and mother. This will mean that the woman must not be laid supine (risk of obstruction to major blood vessels) or prone (risk to fetus). Any unit that has a pregnant woman admitted should have large beanbags available so that the woman can be lowered into the beanbag and therefore retain a semi-seated 
position where she is supported.

- When choosing an agent for rapid tranquillization in a pregnant woman, an antipsychotic or a benzodiazepine with a short half-life should be considered.

- If an antipsychotic is used, it should be at the minimum effective dose because of the risk of neonatal extra pyramidal symptoms.

- If a benzodiazepine is used, the risks of floppy baby syndrome should be taken into account.

- Decisions on choice of medication should be made on an individual basis taking into account both the risks to the mother and to the fetus.

- Intramuscular injections for rapid tranquilization may be administered into the gluteal muscle or the lateral thigh.

- After rapid tranquillization the woman must be reviewed by a midwife to look for signs:

- that the fetus may have been harmed by the procedure.

- that the mother is in labor, as rapid deterioration in mental state towards the end of pregnancy may indicate that the mother is going into labor.

- During labor, if the woman becomes acutely disturbed, her care should be managed in close collaboration with midwifery, a paediatrician, an anaesthetist and psychiatry. All interventions should be documented and communicated verbally in handover to all professionals.

- If the woman is acutely disturbed in the postnatal period it is important that another adult has responsibility for caring for the infant.

- Clinicians must hold in mind that if the mother's symptomatology incorporates the infant and the infant is removed from her or kept at a distance from her, then she may become even more agitated and distressed.

- Clinicians on psychiatric Mother and Baby Units and acute wards that admit pregnant women should develop competence through role play, discussion and multi-disciplinary learning in managing the acutely disturbed pregnant and postnatal woman. Care plans must recognize that a woman may become acutely disturbed. 
- The teams providing care must ensure that the woman is kept hydrated, and that observations of temperature, pulse, $B P$, respiratory rate and oximetry are carried out regularly and documented.

- Over-sedation has particular risks for the pregnant and postnatal woman particularly if she resumes care of her infant. Effects on the fetus of drug exposure through the placenta or to the infant in breastmilk must be considered and appropriate precautions taken.

\section{ACKNOWLEDGEMENTS, FUNDING AND DECLARATION OF INTEREST}

\subsection{Acknowledgements}

Special thanks are due to Susan Chandler who organized the logistics of the consensus meeting and managed the process of the generation of the guidelines. RHMW, IJ, CP, RC planned the meeting. All of the authors contributed text for the guidelines. RHMW edited the manuscript. We would like to thank additional members of the consensus group: J. Cranston, C. Henshaw, A. Kent, J. Rankin, C. Rose. We would also like to thank A. Cairns for comments on the manuscript. All authors approved the final draft.

\subsection{Funding}

The authors received no financial support for the research, authorship, and/or publication of this article. Travel and accommodation costs for attending the consensus meeting were reimbursed by the BAP where necessary.

\subsection{Declaration of Interests}

For disclosure of competing interests for all authors, visit http://www. bap.org.uk/perinataldeclarations 


\section{REFERENCES}

Abel E L, Hannigan J H (1995) Maternal risk factors in fetal alcohol syndrome: provocative and permissive influences. Neurotoxicol Teratol 17: 445-462

Abel K M, Howard L M (2014) Schizophrenia, psychopharmacology and pregnancy. $119-138$

Ackerman J P, Riggins T, Black M M (2010) A review of the effects of prenatal cocaine exposure among school-aged children. Pediatrics 125: 554-565

Akasheh G, Sirati L, Noshad Kamran A R, Sepehrmanesh Z (2014) Comparison of the effect of sertraline with behavioral therapy on semen parameters in men with primary premature ejaculation. Urology 83: 800-804

Alder J, Fink N, Bitzer J, Hosli I, Holzgreve W (2007) Depression and anxiety during pregnancy: a risk factor for obstetric, fetal and neonatal outcome? A critical review of the literature. J Matern Fetal Neonatal Med 20: 189-209

Alwan S, Reefhuis J, Botto L D, Rasmussen S A, Correa A, Friedman J M (2010) Maternal use of bupropion and risk for congenital heart defects. Am J Obstet Gynecol 203: $52-56$

Anderson E L, Reti I M (2009) ECT in pregnancy: a review of the literature from 1941 to 2007. Psychosom Med 71: 235-242

Baldwin D S, Anderson I M, Nutt D J, Allgulander C, Bandelow B, den Boer J A, Christmas D M, Davies S, Fineberg N, Lidbetter N, Malizia A, McCrone P, Nabarro D, O'Neill C, Scott J, van der Wee N, Wittchen H U (2014) Evidence-based pharmacological treatment of anxiety disorders, post-traumatic stress disorder and obsessive-compulsive disorder: a revision of the 2005 guidelines from the British Association for Psychopharmacology. J Psychopharmacol 28: 403-439

Ban L, Fleming K M, Doyle P, Smeeth L, Hubbard R B, Fiaschi L, Tata L J (2015) Congenital Anomalies in Children of Mothers Taking Antiepileptic Drugs with and without Periconceptional High Dose Folic Acid Use: A Population-Based Cohort Study. PLoS One 10: e0131130-

Ban L, West J, Gibson J E, Fiaschi L, Sokal R, Doyle P, Hubbard R, Smeeth L, Tata L J (2014) First trimester exposure to anxiolytic and hypnotic drugs and the risks of major congenital anomalies: a United Kingdom population-based cohort study. PLoS One 9: e100996-

Bateman B T, Patorno E, Desai R J, Seely E W, Mogun H, Maeda A, Fischer M A, Hernandez-Diaz S, Huybrechts K F (2016) Late Pregnancy beta Blocker Exposure and Risks of Neonatal Hypoglycemia and Bradycardia. Pediatrics 138:

BC Reproductive Mental Health Programme and Perinatal Services BC (2014) Best practice guidelines for mental health disorders in the perinatal period. 
Bech B H, Kjaersgaard M I, Pedersen H S, Howards P P, Sorensen M J, Olsen J, Parner E T, Pedersen L H, Vestergaard M, Christensen J (2014) Use of antiepileptic drugs during pregnancy and risk of spontaneous abortion and stillbirth: population based cohort study. Brit Med J 349: g5159-

Bennedsen B E, Mortensen P B, Olesen A V, Henriksen T B (1999) Preterm birth and intra-uterine growth retardation among children of women with schizophrenia. $\mathrm{Br} \mathrm{J}$ Psychiatry 175: 239-245

Bennedsen B E, Mortensen P B, Olesen A V, Henriksen T B (2001) Congenital malformations, stillbirths, and infant deaths among children of women with schizophrenia. Arch Gen Psychiatry 58: 674-679

Berard A, Zhao J P, Sheehy O (2016) Success of smoking cessation interventions during pregnancy. Am J Obstet Gynecol

Bergman K, Sarkar P, O'Connor T G, Modi N, Glover V (2007) Maternal stress during pregnancy predicts cognitive ability and fearfulness in infancy. $\mathrm{J}$ Am Acad Child Adolesc Psychiatry 46: 1454-1463

Berle J O, Spigset O (2011) Antidepressant Use During Breastfeeding. Curr Womens Health Rev 7: 28-34

Besag F M (2014) ADHD treatment and pregnancy. Drug Saf 37: 397-408

Blais M A, Becker A E, Burwell R A, Flores A T, Nussbaum K M, Greenwood D N, Ekeblad E R, Herzog D B (2000) Pregnancy: outcome and impact on symptomatology in a cohort of eating-disordered women. Int J Eat Disord 27: 140-149

Boden R, Lundgren M, Brandt L, Reutfors J, Andersen M, Kieler H (2012a) Risks of adverse pregnancy and birth outcomes in women treated or not treated with mood stabilisers for bipolar disorder: population based cohort study. Brit Med J 345: e7085-

Boden R, Lundgren M, Brandt L, Reutfors J, Kieler H (2012b) Antipsychotics during pregnancy: relation to fetal and maternal metabolic effects. Arch Gen Psychiatry 69: 715-721

Boden R, Lundgren M, Brandt L, Reutfors J, Kieler H (2012c) Antipsychotics during pregnancy: relation to fetal and maternal metabolic effects. Arch Gen Psychiatry 69: 715-721

Bogen D L, Sit D, Genovese A, Wisner K L (2012) Three cases of lithium exposure and exclusive breastfeeding. Arch Womens Ment Health 15: 69-72

Bolea-Alamanac B M, Green A, Verma G, Maxwell P, Davies S J (2014)

Methylphenidate use in pregnancy and lactation: a systematic review of evidence. Br J Clin Pharmacol 77: 96-101

Boukhris T, Sheehy O, Mottron L, Berard A (2016) Antidepressant Use During Pregnancy and the Risk of Autism Spectrum Disorder in Children. JAMA Pediatr 170: 117-124 
Boyle B, Garne E, Loane M, Addor M C, Arriola L, Cavero-Carbonell C, Gatt M, Lelong N, Lynch C, Nelen V, Neville A J, O'Mahony M, Pierini A, Rissmann A, Tucker D, Zymak-Zakutnia N, Dolk H (2016) The changing epidemiology of Ebstein's anomaly and its relationship with maternal mental health conditions: a European registry-based study. Cardiol Young 1-9

Bradley R, Slade P (2011) A review of mental health problems in fathers following the birth of a child. Journal of Reproductive and Infant Psychology 29: 19-42

Bricelj V (1999) Use of Adrenergic Beta-Blockers in Pregnancy. Heart Views 1: 130132

Bro S P, Kjaersgaard M I, Parner E T, Sorensen M J, Olsen J, Bech B H, Pedersen L H, Christensen J, Vestergaard M (2015) Adverse pregnancy outcomes after exposure to methylphenidate or atomoxetine during pregnancy. Clin Epidemiol 7: 139-147

Bromley R, Weston J, Adab N, Greenhalgh J, Sanniti A, McKay A J, Tudur S C, Marson A G (2014) Treatment for epilepsy in pregnancy: neurodevelopmental outcomes in the child. Cochrane Database Syst Rev CD010236-

Bruning A H, Heller H M, Kieviet N, Bakker P C, de Groot C J, Dolman K M, Honig A (2015) Antidepressants during pregnancy and postpartum hemorrhage: a systematic review. Eur J Obstet Gynecol Reprod Biol 189: 38-47

Brunton R J, Dryer R, Saliba A, Kohlhoff J (2015) Pregnancy anxiety: A systematic review of current scales. J Affect Disord 176: 24-34

Bulik C M, Hoffman E R, Von H A, Torgersen L, Stoltenberg C, ReichbornKjennerud $\mathrm{T}$ (2010) Unplanned pregnancy in women with anorexia nervosa. Obstet Gynecol 116: 1136-1140

Bulik C M, Von H A, Hamer R, Knoph B C, Torgersen L, Magnus P, Stoltenberg C, Siega-Riz A M, Sullivan P, Reichborn-Kjennerud T (2007) Patterns of remission, continuation and incidence of broadly defined eating disorders during early pregnancy in the Norwegian Mother and Child Cohort Study (MoBa). Psychol Med 37: 11091118

Burke L (2003) The impact of maternal depression on familial relationships. Int Rev Psychiatry 15: 243-255

Burns L, Mattick R P, Lim K, Wallace C (2007) Methadone in pregnancy: treatment retention and neonatal outcomes. Addiction 102: 264-270

Cannon M, Jones P B, Murray R M (2002) Obstetric complications and schizophrenia: historical and meta-analytic review. Am J Psychiatry 159: 1080-1092

Cantwell R, Clutton-Brock T, Cooper G, Dawson A, Drife J, Garrod D, Harper A, Hulbert D, Lucas S, McClure J, Millward-Sadler H, Neilson J, Nelson-Piercy C, Norman J, O'Herlihy C, Oates M, Shakespeare J, de S M, Williamson C, Beale V, Knight M, Lennox C, Miller A, Parmar D, Rogers J, Springett A (2011) Saving Mothers' Lives: Reviewing maternal deaths to make motherhood safer: 2006-2008. 
The Eighth Report of the Confidential Enquiries into Maternal Deaths in the United Kingdom. BJOG 118 Suppl 1: 1-203

Castro V M, Kong S W, Clements C C, Brady R, Kaimal A J, Doyle A E, Robinson E B, Churchill S E, Kohane I S, Perlis R H (2016) Absence of evidence for increase in risk for autism or attention-deficit hyperactivity disorder following antidepressant exposure during pregnancy: a replication study. Transl Psychiatry 6: e708-

Caton A R, Bell E M, Druschel C M, Werler M M, Lin A E, Browne M L, McNutt L A, Romitti P A, Mitchell A A, Olney R S, Correa A (2009) Antihypertensive medication use during pregnancy and the risk of cardiovascular malformations. Hypertension 54: 63-70

Center for Substance Abuse Treatment (2008) Medication-assisted treatment for opioid addiction during pregnancy.

Chamberlain C, O'Mara-Eves A, Oliver S, Caird J R, Perlen S M, Eades S J, Thomas J (2013) Psychosocial interventions for supporting women to stop smoking in pregnancy. Cochrane Database Syst Rev CD001055-

Chambers C D, Hernandez-Diaz S, Van Marter L J, Werler M M, Louik C, Jones K L, Mitchell A A (2006) Selective serotonin-reuptake inhibitors and risk of persistent pulmonary hypertension of the newborn. N Engl J Med 354: 579-587

Chomchai C, Na M N, Watanarungsan P, Yossuck P, Chomchai S (2004)

Methamphetamine abuse during pregnancy and its health impact on neonates born at Siriraj Hospital, Bangkok, Thailand. Southeast Asian J Trop Med Public Health 35: 228-231

Christensen J, Gronborg T K, Sorensen M J, Schendel D, Parner E T, Pedersen L H, Vestergaard M (2013) Prenatal valproate exposure and risk of autism spectrum disorders and childhood autism. JAMA 309: 1696-1703

Chun-Fai-Chan B, Koren G, Fayez I, Kalra S, Voyer-Lavigne S, Boshier A, Shakir S, Einarson A (2005) Pregnancy outcome of women exposed to bupropion during pregnancy: a prospective comparative study. Am J Obstet Gynecol 192: 932-936

Chung T K, Lau T K, Yip A S, Chiu H F, Lee D T (2001) Antepartum depressive symptomatology is associated with adverse obstetric and neonatal outcomes. Psychosom Med 63: 830-834

Cleare A, Pariante C M, Young A H, Anderson I M, Christmas D, Cowen P J, Dickens C, Ferrier I N, Geddes J, Gilbody S, Haddad P M, Katona C, Lewis G, Malizia A, McAllister-Williams R H, Ramchandani P, Scott J, Taylor D, Uher R (2015) Evidence-based guidelines for treating depressive disorders with antidepressants: A revision of the 2008 British Association for Psychopharmacology guidelines. J Psychopharmacol 29: 459-525

Clements C C, Castro V M, Blumenthal S R, Rosenfield H R, Murphy S N, Fava M, Erb J L, Churchill S E, Kaimal A J, Doyle A E, Robinson E B, Smoller J W, Kohane I 
S, Perlis R H (2015) Prenatal antidepressant exposure is associated with risk for attention-deficit hyperactivity disorder but not autism spectrum disorder in a large health system. Mol Psychiatry 20: 727-734

Cohen L S, Altshuler L L, Harlow B L, Nonacs R, Newport D J, Viguera A C, Suri R, Burt V K, Hendrick V, Reminick A M, Loughead A, Vitonis A F, Stowe Z N (2006) Relapse of major depression during pregnancy in women who maintain or discontinue antidepressant treatment. JAMA 295: 499-507

Cohen L S, Nonacs R M, Bailey J W, Viguera A C, Reminick A M, Altshuler L L, Stowe Z N, Faraone S V (2004) Relapse of depression during pregnancy following antidepressant discontinuation: a preliminary prospective study. Arch Womens Ment Health 7: 217-221

Cohen L S, Viguera A C, McInerney K A, Freeman M P, Sosinsky A Z, Moustafa D, Marfurt S P, Kwiatkowski M A, Murphy S K, Farrell A M, Chitayat D, HernandezDiaz S (2016) Reproductive Safety of Second-Generation Antipsychotics: Current Data From the Massachusetts General Hospital National Pregnancy Registry for Atypical Antipsychotics. Am J Psychiatry 173: 263-270

Coleman T, Chamberlain C, Davey M A, Cooper S E, Leonardi-Bee J (2015) Pharmacological interventions for promoting smoking cessation during pregnancy. Cochrane Database Syst Rev CD010078-

Coughlin C G, Blackwell K A, Bartley C, Hay M, Yonkers K A, Bloch M H (2015) Obstetric and neonatal outcomes after antipsychotic medication exposure in pregnancy. Obstet Gynecol 125: 1224-1235

Croen L A, Grether J K, Yoshida C K, Odouli R, Hendrick V (2011) Antidepressant use during pregnancy and childhood autism spectrum disorders. Arch Gen Psychiatry 68: $1104-1112$

Crow S J, Agras W S, Crosby R, Halmi K, Mitchell J E (2008) Eating disorder symptoms in pregnancy: a prospective study. Int J Eat Disord 41: 277-279

Dalman C, Allebeck P, Cullberg J, Grunewald C, Koster M (1999) Obstetric complications and the risk of schizophrenia: a longitudinal study of a national birth cohort. Arch Gen Psychiatry 56: 234-240

Darwish M, Martin P T, Cevallos W H, Tse S, Wheeler S, Troy S M (1999) Rapid disappearance of zaleplon from breast milk after oral administration to lactating women. J Clin Pharmacol 39: 670-674

Davalos D B, Yadon C A, Tregellas H C (2012) Untreated prenatal maternal depression and the potential risks to offspring: a review. Arch Womens Ment Health 15: $1-14$

Davanzo R, Copertino M, De C A, Minen F, Amaddeo A (2011) Antidepressant drugs and breastfeeding: a review of the literature. Breastfeed Med 6: 89-98 
Davis R L, Eastman D, McPhillips H, Raebel M A, Andrade S E, Smith D, Yood M U, Dublin S, Platt R (2011) Risks of congenital malformations and perinatal events among infants exposed to calcium channel and beta-blockers during pregnancy. Pharmacoepidemiol Drug Saf 20: 138-145

De Asis S J, Helgeson L, Ostroff R (2013) The use of propofol to prevent fetal deceleration during electroconvulsive therapy treatment. J ECT 29: e57-e58

De R M, Zarrilli S, Di S A, Milano N, Gaccione M, Boggia B, Lombardi G, Colao A (2003) Hyperprolactinemia in men: clinical and biochemical features and response to treatment. Endocrine 20: 75-82

Debooy V D, Seshia M M, Tenenbein M, Casiro O G (1993) Intravenous pentazocine and methylphenidate abuse during pregnancy. Maternal lifestyle and infant outcome. Am J Dis Child 147: 1062-1065

DeVido J, Bogunovic O, Weiss R D (2015) Alcohol use disorders in pregnancy. Harv Rev Psychiatry 23: 112-121

Diav-Citrin O, Shechtman S, Arnon J, Wajnberg R, Borisch C, Beck E, Richardson J L, Bozzo P, Nulman I, Ornoy A (2016) Methylphenidate in Pregnancy: A Multicenter, Prospective, Comparative, Observational Study. J Clin Psychiatry 77: 1176-1181

Diav-Citrin O, Shechtman S, Ornoy S, Arnon J, Schaefer C, Garbis H, Clementi M, Ornoy A (2005) Safety of haloperidol and penfluridol in pregnancy: a multicenter, prospective, controlled study. J Clin Psychiatry 66: 317-322

Diav-Citrin O, Shechtman S, Tahover E, Finkel-Pekarsky V, Arnon J, Kennedy D, Erebara A, Einarson A, Ornoy A (2014) Pregnancy outcome following in utero exposure to lithium: a prospective, comparative, observational study. Am J Psychiatry 171: 785-794

Diego M A, Field T, Hernandez-Reif M, Schanberg S, Kuhn C, Gonzalez-Quintero V H (2009) Prenatal depression restricts fetal growth. Early Hum Dev 85: 65-70

Dolk H, Wang H, Loane M, Morris J, Garne E, Addor M C, Arriola L, Bakker M, Barisic I, Doray B, Gatt M, Kallen K, Khoshnood B, Klungsoyr K, LahesmaaKorpinen A M, Latos-Bielenska A, Mejnartowicz J P, Nelen V, Neville A, O'Mahony M, Pierini A, Rissmann A, Tucker D, Wellesley D, Wiesel A, de Jong-van den Berg LT (2016) Lamotrigine use in pregnancy and risk of orofacial cleft and other congenital anomalies. Neurology 86: 1716-1725

Dolovich L R, Addis A, Vaillancourt J M, Power J D, Koren G, Einarson T R (1998) Benzodiazepine use in pregnancy and major malformations or oral cleft: meta-analysis of cohort and case-control studies. Brit Med J 317: 839-843

Easter A, Bye A, Taborelli E, Corfield F, Schmidt U, Treasure J, Micali N (2013) Recognising the symptoms: how common are eating disorders in pregnancy? Eur Eat Disord Rev 21: 340-344 
Easter A, Solmi F, Bye A, Taborelli E, Corfield F, Schmidt U, Treasure J, Micali N (2015) Antenatal and postnatal psychopathology among women with current and past eating disorders: longitudinal patterns. Eur Eat Disord Rev 23: 19-27

Easter A, Treasure J, Micali N (2011) Fertility and prenatal attitudes towards pregnancy in women with eating disorders: results from the Avon Longitudinal Study of Parents and Children. BJOG 118: 1491-1498

El M H, White T, Verhulst F C, Tiemeier H (2014) Maternal use of antidepressant or anxiolytic medication during pregnancy and childhood neurodevelopmental outcomes: a systematic review. Eur Child Adolesc Psychiatry 23: 973-992

Ennis Z N, Damkier P (2015) Pregnancy exposure to olanzapine, quetiapine, risperidone, aripiprazole and risk of congenital malformations. A systematic review. Basic Clin Pharmacol Toxicol 116: 315-320

Eryilmaz G, Sayar G H, Ozten E, Gul I G, Yorbik O, Isiten N, Bagci E (2015) Followup study of children whose mothers were treated with transcranial magnetic stimulation during pregnancy: preliminary results. Neuromodulation 18: 255-260

Field T, Diego M, Hernandez-Reif M (2006) Prenatal depression effects on the fetus and newborn: a review. Infant Behav Dev 29: 445-455

Field T, Diego M, Hernandez-Reif M, Figueiredo B, Schanberg S, Kuhn C, Deeds O, Contogeorgos J, Ascencio A (2008) Chronic prenatal depression and neonatal outcome. Int J Neurosci 118: 95-103

Figueroa R (2010) Use of antidepressants during pregnancy and risk of attentiondeficit/hyperactivity disorder in the offspring. J Dev Behav Pediatr 31: 641-648

Fontein-Kuipers Y J, Nieuwenhuijze M J, Ausems M, Bude L, de V R (2014) Antenatal interventions to reduce maternal distress: a systematic review and metaanalysis of randomised trials. BJOG 121: 389-397

Forray A, Foster D (2015) Substance Use in the Perinatal Period. Curr Psychiatry Rep 17: 91-

Fox C H (1994) Cocaine use in pregnancy. J Am Board Fam Pract 7: 225-228

Frank D A, Augustyn M, Knight W G, Pell T, Zuckerman B (2001) Growth, development, and behavior in early childhood following prenatal cocaine exposure: a systematic review. JAMA 285: 1613-1625

Franko D L, Blais M A, Becker A E, Delinsky S S, Greenwood D N, Flores A T, Ekeblad E R, Eddy K T, Herzog D B (2001) Pregnancy complications and neonatal outcomes in women with eating disorders. Am J Psychiatry 158: 1461-1466

Freizinger M, Franko D L, Dacey M, Okun B, Domar A D (2010) The prevalence of eating disorders in infertile women. Fertil Steril 93: 72-78 
Geddes J R, Gardiner A, Rendell J, Voysey M, Tunbridge E, Hinds C, Yu L M, Hainsworth J, Attenburrow M J, Simon J, Goodwin G M, Harrison P J (2016)

Comparative evaluation of quetiapine plus lamotrigine combination versus quetiapine monotherapy (and folic acid versus placebo) in bipolar depression (CEQUEL): a 2 × 2 factorial randomised trial. Lancet Psychiatry 3: 31-39

Gentile S (2008) Infant safety with antipsychotic therapy in breast-feeding: a systematic review. J Clin Psychiatry 69: 666-673

Gentile S (2010) Antipsychotic therapy during early and late pregnancy. A systematic review. Schizophr Bull 36: 518-544

Glasheen C, Richardson G A, Fabio A (2010) A systematic review of the effects of postnatal maternal anxiety on children. Arch Womens Ment Health 13: 61-74

Glover V (2014) Maternal depression, anxiety and stress during pregnancy and child outcome; what needs to be done. Best Pract Res Clin Obstet Gynaecol 28: 25-35

Goodman J H (2009) Women's attitudes, preferences, and perceived barriers to treatment for perinatal depression. Birth 36: 60-69

Goodwin G M, Haddad P M, Ferrier I N, Aronson J K, Barnes T R H, Cipriani A, Coghill D R, Fazel S, Geddes J R, Grunze H, Holmes E A, Howes O, Hudson S, Hunt N, Jones I, Macmillan I C, McAllister-Williams R H, Miklowitz D M, Morriss R, Munafo M, Paton C, Saharkian B J, Saunders K E A, Sinclair J M A, Taylor D, Vieta E, Young A H (2016) Evidence-based guidelines for treating bipolar disorder: revised third edition. Recommendations from the British Association for Psychopharmacology. J Psychopharm

Goodwin R D, Keyes K, Simuro N (2007) Mental disorders and nicotine dependence among pregnant women in the United States. Obstet Gynecol 109: 875-883

Gray T R, Choo R E, Concheiro M, Williams E, Elko A, Jansson L M, Jones H E, Huestis M A (2010) Prenatal methadone exposure, meconium biomarker concentrations and neonatal abstinence syndrome. Addiction 105: 2151-2159

Grigoriadis S, VonderPorten E H, Mamisashvili L, Eady A, Tomlinson G, Dennis C L, Koren G, Steiner M, Mousmanis P, Cheung A, Ross L E (2013) The effect of prenatal antidepressant exposure on neonatal adaptation: a systematic review and meta-analysis. J Clin Psychiatry 74: e309-e320

Grote N K, Bridge J A, Gavin A R, Melville J L, Iyengar S, Katon W J (2010) A meta-analysis of depression during pregnancy and the risk of preterm birth, low birth weight, and intrauterine growth restriction. Arch Gen Psychiatry 67: 1012-1024

Grzeskowiak L E, Morrison J L, Henriksen T B, Bech B H, Obel C, Olsen J, Pedersen L H (2015) Prenatal antidepressant exposure and child behavioural outcomes at 7 years of age: a study within the Danish National Birth Cohort. BJOG 
Habermann F, Fritzsche J, Fuhlbruck F, Wacker E, Allignol A, Weber-Schoendorfer C, Meister R, Schaefer C (2013) Atypical antipsychotic drugs and pregnancy outcome: a prospective, cohort study. J Clin Psychopharmacol 33: 453-462

Haddad P M, Talbot P S, Anderson I M, McAllister-Williams R H (2015) Managing inadequate antidepressant response in depressive illness. Br Med Bull 115: 183-201

Haddad P M, Wieck A (2004) Antipsychotic-induced hyperprolactinaemia: mechanisms, clinical features and management. Drugs 64: 2291-2314

Haervig K B, Mortensen L H, Hansen A V, Strandberg-Larsen K (2014) Use of ADHD medication during pregnancy from 1999 to 2010: a Danish register-based study. Pharmacoepidemiol Drug Safety 23: 526-533

Hamed S A, Moussa E M, Tohamy A M, Mohamed K O, Mohamad M E, Sherif T M, Abdellah M M (2015) Seminal fluid analysis and testicular volume in adults with epilepsy receiving valproate. J Clin Neurosci 22: 508-512

Hanley G E, Smolina K, Mintzes B, Oberlander T F, Morgan S G (2016) Postpartum Hemorrhage and Use of Serotonin Reuptake Inhibitor Antidepressants in Pregnancy. Obstet Gynecol 127: 553-561

Hartz S C, Heinonen O P, Shapiro S, Siskind V, Slone D (1975) Antenatal exposure to meprobamate and chlordiazepoxide in relation to malformations, mental development, and childhood mortality. N Engl J Med 292: 726-728

Hay D F, Pawlby S, Waters C S, Sharp D (2008) Antepartum and postpartum exposure to maternal depression: different effects on different adolescent outcomes. J Child Psychol Psychiatry 49: 1079-1088

Hizli S G, Ozten E, Tufan E, Cerit C, Kagan G, Dilbaz N, Tarhan N (2014) Transcranial magnetic stimulation during pregnancy. Arch Womens Ment Health 17: 311-315

Houser M V, Hennessy M D, Howard B C (2010) Vagal nerve stimulator use during pregnancy for treatment of refractory seizure disorder. Obstet Gynecol 115: 417-419

Howard L, Bekele D, Rowe M, Demilew J, Bewley S, Marteau T (2013) Smoking cessation in pregnant women with mental disorders: a cohort and nested qualitative study. BJOG 120: 362-370

Howard L M (2005) Fertility and pregnancy in women with psychotic disorders. Eur J Obstet Gynecol Reprod Biol 119: 3-10

Howard L M, Goss C, Leese M, Thornicroft G (2003) Medical outcome of pregnancy in women with psychotic disorders and their infants in the first year after birth. Br J Psychiatry 182: 63-67

Hu X, Wang J, Dong W, Fang Q, Hu L, Liu C (2011) A meta-analysis of polycystic ovary syndrome in women taking valproate for epilepsy. Epilepsy Res 97: 73-82 
Husain M M, Stegman D, Trevino K (2005) Pregnancy and delivery while receiving vagus nerve stimulation for the treatment of major depression: a case report. Ann Gen Psychiatry 4: 16-

Huybrechts K F, Bateman B T, Palmsten K, Desai R J, Patorno E, Gopalakrishnan C, Levin R, Mogun H, Hernandez-Diaz S (2015) Antidepressant use late in pregnancy and risk of persistent pulmonary hypertension of the newborn. JAMA 313: 2142-2151

Huybrechts K F, Hernandez-Diaz S, Avorn J (2014a) Antidepressant use in pregnancy and the risk of cardiac defects. N Engl J Med 371: 1168-1169

Huybrechts K F, Hernandez-Diaz S, Avorn J (2014b) Antidepressant use in pregnancy and the risk of cardiac defects. N Engl J Med 371: 1168-1169

Huybrechts K F, Hernandez-Diaz S, Patorno E, Desai R J, Mogun H, Dejene S Z, Cohen J M, Panchaud A, Cohen L, Bateman B T (2016) Antipsychotic Use in Pregnancy and the Risk for Congenital Malformations. JAMA Psychiatry 73: 938-946

Ito S, Blajchman A, Stephenson M, Eliopoulos C, Koren G (1993) Prospective follow-up of adverse reactions in breast-fed infants exposed to maternal medication. Am J Obstet Gynecol 168: 1393-1399

Jablensky A V, Morgan V, Zubrick S R, Bower C, Yellachich L A (2005) Pregnancy, delivery, and neonatal complications in a population cohort of women with schizophrenia and major affective disorders. Am J Psychiatry 162: 79-91

Jentink J, Dolk H, Loane M A, Morris J K, Wellesley D, Garne E, de Jong-van den Berg (2010a) Intrauterine exposure to carbamazepine and specific congenital malformations: systematic review and case-control study. Brit Med J 341: c6581-

Jentink J, Loane M A, Dolk H, Barisic I, Garne E, Morris J K, de Jong-van den Berg LT (2010b) Valproic acid monotherapy in pregnancy and major congenital malformations. N Engl J Med 362: 2185-2193

Johnson K C, LaPrairie J L, Brennan P A, Stowe Z N, Newport D J (2012) Prenatal antipsychotic exposure and neuromotor performance during infancy. Arch Gen Psychiatry 69: 787-794

Jones H E, Kaltenbach K, Heil S H, Stine S M, Coyle M G, Arria A M, O'Grady K E, Selby P, Martin P R, Fischer G (2010) Neonatal abstinence syndrome after methadone or buprenorphine exposure. N Engl J Med 363: 2320-2331

Jones I, Chandra P S, Dazzan P, Howard L M (2014) Bipolar disorder, affective psychosis, and schizophrenia in pregnancy and the post-partum period. Lancet 384 : 1789-1799

Juric S, Newport D J, Ritchie J C, Galanti M, Stowe Z N (2009) Zolpidem (Ambien) in pregnancy: placental passage and outcome. Arch Womens Ment Health 12: 441446 
Kallen B, Borg N, Reis M (2013) The use of central nervous system active drugs during pregnancy. Pharmaceuticals (Basel) 6: 1221-1286

Kallen B A, Otterblad O P (2007) Maternal use of selective serotonin re-uptake inhibitors in early pregnancy and infant congenital malformations. Birth Defects Res A Clin Mol Teratol 79: 301-308

Kaplan Y C, Keskin-Arslan E, Acar S, Sozmen K (2016) Prenatal selective serotonin reuptake inhibitor use and the risk of autism spectrum disorder in children: A systematic review and meta-analysis. Reprod Toxicol 66: 31-43

Kelly L E, Poon S, Madadi P, Koren G (2012) Neonatal benzodiazepines exposure during breastfeeding. J Pediatr 161: 448-451

Khoshnood B, Loane M, de W H, Arriola L, Addor M C, Barisic I, Beres J, Bianchi F, Dias C, Draper E, Garne E, Gatt M, Haeusler M, Klungsoyr K, Latos-Bielenska A, Lynch C, McDonnell B, Nelen V, Neville A J, O'Mahony M T, Queisser-Luft A, Rankin J, Rissmann A, Ritvanen A, Rounding C, Sipek A, Tucker D, VerellenDumoulin C, Wellesley D, Dolk H (2015) Long term trends in prevalence of neural tube defects in Europe: population based study. Brit Med J 351: h5949-

Kim D R, Epperson N, Pare E, Gonzalez J M, Parry S, Thase M E, Cristancho P, Sammel M D, O'Reardon J P (2011a) An open label pilot study of transcranial magnetic stimulation for pregnant women with major depressive disorder. J Womens Health (Larchmt ) 20: 255-261

Kim D R, Snell J L, Ewing G C, O'Reardon J (2015) Neuromodulation and antenatal depression: a review. Neuropsychiatr Dis Treat 11: 975-982

Kim D R, Sockol L, Barber J P, Moseley M, Lamprou L, Rickels K, O'Reardon J P, Epperson C N (2011b) A survey of patient acceptability of repetitive transcranial magnetic stimulation (TMS) during pregnancy. J Affect Disord 129: 385-390

Kim J J, Silver R K (2016) Perinatal suicide associated with depression diagnosis and absence of active treatment in 15-year UK national inquiry. Evid Based Ment Health 19: 122-

King-Hele S, Webb R T, Mortensen P B, Appleby L, Pickles A, Abel K M (2009) Risk of stillbirth and neonatal death linked with maternal mental illness: a national cohort study. Arch Dis Child Fetal Neonatal Ed 94: F105-F110

Kjaersgaard M I, Parner E T, Vestergaard M, Sorensen M J, Olsen J, Christensen J, Bech B H, Pedersen L H (2013) Prenatal antidepressant exposure and risk of spontaneous abortion - a population-based study. PLoS One 8: e72095-

Knapp W P, Soares B G, Farrel M, Lima M S (2007) Psychosocial interventions for cocaine and psychostimulant amphetamines related disorders. Cochrane Database Syst Rev CD003023-

Knight M, Tuffnell D, Kenyon S, Shakespeare J, Gray R, Kurinczuk J J, on behalf of MBRRACE-UK (2015) Saving Lives, Improving Mothers' Care - Surveillance of 
maternal deaths in the UK 2011-13 and lessons learned to inform maternity care from the UK and Ireland Confidential Enquiries into Maternal Deaths and Morbidity 200913.

Kraljevic M, Warnock F F (2013) Early educational and behavioral RCT interventions to reduce maternal symptoms of psychological trauma following preterm birth: a systematic review. J Perinat Neonatal Nurs 27: 311-327

Kuhn L, Kline J, Ng S, Levin B, Susser M (2000) Cocaine use during pregnancy and intrauterine growth retardation: new insights based on maternal hair tests. Am J Epidemiol 152: 112-119

Laegreid L, Hagberg G, Lundberg A (1992) Neurodevelopment in late infancy after prenatal exposure to benzodiazepines--a prospective study. Neuropediatrics 23: 60-67

Lancaster C A, Gold K J, Flynn H A, Yoo H, Marcus S M, Davis M M (2010) Risk factors for depressive symptoms during pregnancy: a systematic review. Am J Obstet Gynecol 202: 5-14

Langley K, Heron J, Smith G D, Thapar A (2012) Maternal and paternal smoking during pregnancy and risk of ADHD symptoms in offspring: testing for intrauterine effects. Am J Epidemiol 176: 261-268

Larsen E R, Damkier P, Pedersen L H, Fenger-Gron J, Mikkelsen R L, Nielsen R E, Linde V J, Knudsen H E, Skaarup L, Videbech P (2015) Use of psychotropic drugs during pregnancy and breast-feeding. Acta Psychiatr Scand Suppl 1-28

Laugesen K, Olsen M S, Telen Andersen A B, Froslev T, Sorensen H T (2013) In utero exposure to antidepressant drugs and risk of attention deficit hyperactivity disorder: a nationwide Danish cohort study. BMJ Open 3: e003507-

Lawson A, Murphy K E, Sloan E, Uleryk E, Dalfen A (2015) The relationship between sleep and postpartum mental disorders: A systematic review. J Affect Disord 176: $65-77$

Leiknes K A, Cooke M J, Jarosch-von S L, Harboe I, Hoie B (2015)

Electroconvulsive therapy during pregnancy: a systematic review of case studies. Arch Womens Ment Health 18: 1-39

Li D, Liu L, Odouli R (2009) Presence of depressive symptoms during early pregnancy and the risk of preterm delivery: a prospective cohort study. Hum Reprod 24: $146-153$

Lin H C, Chen I J, Chen Y H, Lee H C, Wu F J (2010) Maternal schizophrenia and pregnancy outcome: does the use of antipsychotics make a difference? Schizophr Res 116: $55-60$

Lin S, Leonard D, Co M A, Mukhopadhyay D, Giri B, Perger L, Beeram M R, Kuehl T J, Uddin M N (2015) Pre-eclampsia has an adverse impact on maternal and fetal health. Transl Res 165: 449-463 
Lingford-Hughes A R, Welch S, Peters L, Nutt D J (2012) BAP updated guidelines: evidence-based guidelines for the pharmacological management of substance abuse, harmful use, addiction and comorbidity: recommendations from BAP. J

Psychopharmacol 26: 899-952

Linna M S, Raevuori A, Haukka J, Suvisaari J M, Suokas J T, Gissler M (2014)

Pregnancy, obstetric, and perinatal health outcomes in eating disorders. Am J Obstet Gynecol 211: 392-398

Louik C, Kerr S, Mitchell A A (2014) First-trimester exposure to bupropion and risk of cardiac malformations. Pharmacoepidemiol Drug Saf 23: 1066-1075

Mactier H, Shipton D, Dryden C, Tappin D M (2014) Reduced fetal growth in methadone-maintained pregnancies is not fully explained by smoking or socioeconomic deprivation. Addiction 109: 482-488

Magee L A, Duley L (2003) Oral beta-blockers for mild to moderate hypertension during pregnancy. Cochrane Database Syst Rev CD002863-

Makarechian N, Agro K, Devlin J, Trepanier E, Koren G, Einarson T R (1998)

Association between moderate alcohol consumption during pregnancy and spontaneous abortion, stillbirth and premature birth: a meta-analysis. Can J Clin Pharmacol 5: 169-176

Malm H, Artama M, Gissler M, Ritvanen A (2011) Selective serotonin reuptake inhibitors and risk for major congenital anomalies. Obstet Gynecol 118: 111-120

Man K K, Tong H H, Wong L Y, Chan E W, Simonoff E, Wong I C (2015) Exposure to selective serotonin reuptake inhibitors during pregnancy and risk of autism spectrum disorder in children: a systematic review and meta-analysis of observational studies. Neurosci Biobehav Rev 49: 82-89

McCorry D, Bromley R (2015) Does in utero exposure of antiepileptic drugs lead to failure to reach full cognitive potential? Seizure 28: 51-56

McGuinness M, Blissett J, Jones C (2011) OCD in the perinatal period: is postpartum OCD (ppOCD) a distinct subtype? A review of the literature. Behav Cogn Psychother 39: $285-310$

McKenna K, Koren G, Tetelbaum M, Wilton L, Shakir S, Diav-Citrin O, Levinson A, Zipursky R B, Einarson A (2005) Pregnancy outcome of women using atypical antipsychotic drugs: a prospective comparative study. J Clin Psychiatry 66: 444-449

McKnight R F, Adida M, Budge K, Stockton S, Goodwin G M, Geddes J R (2012) Lithium toxicity profile: a systematic review and meta-analysis. Lancet 379: 721-728

Meador K, Reynolds M W, Crean S, Fahrbach K, Probst C (2008) Pregnancy outcomes in women with epilepsy: a systematic review and meta-analysis of published pregnancy registries and cohorts. Epilepsy Res 81: 1-13 
Meidahl P K, Jimenez-Solem E, Andersen J T, Petersen M, Brodbaek K, Kober L, Torp-Pedersen C, Poulsen H E (2012) beta-Blocker treatment during pregnancy and adverse pregnancy outcomes: a nationwide population-based cohort study. BMJ Open 2:

Metz T D, Stickrath E H (2015) Marijuana use in pregnancy and lactation: a review of the evidence. Am J Obstet Gynecol 213: 761-778

Micali N, dos-Santos-Silva I, De S B, Steenweg-de G J, Jaddoe V, Hofman A, Verhulst F C, Steegers E, Tiemeier H (2014) Fertility treatment, twin births, and unplanned pregnancies in women with eating disorders: findings from a populationbased birth cohort. BJOG 121: 408-416

Micali N, Hagberg K W, Petersen I, Treasure J L (2013) The incidence of eating disorders in the UK in 2000-2009: findings from the General Practice Research Database. BMJ Open 3:

Micali N, Simonoff E, Stahl D, Treasure J (2011a) Maternal eating disorders and infant feeding difficulties: maternal and child mediators in a longitudinal general population study. J Child Psychol Psychiatry 52: 800-807

Micali N, Simonoff E, Treasure J (2011b) Pregnancy and post-partum depression and anxiety in a longitudinal general population cohort: the effect of eating disorders and past depression. J Affect Disord 131: 150-157

Micali N, Stemann L P, Strandberg-Larsen K, Nybo Andersen A M (2016a) Size at birth and preterm birth in women with lifetime eating disorders: a prospective population-based study. BJOG 123: 1301-1310

Micali N, Stemann L P, Strandberg-Larsen K, Nybo Andersen A M (2016b) Size at birth and preterm birth in women with lifetime eating disorders: a prospective population-based study. BJOG 123: 1301-1310

Miller L J (1994) Use of electroconvulsive therapy during pregnancy. Hosp Community Psychiatry 45: 444-450

Minozzi S, Amato L, Bellisario C, Ferri M, Davoli M (2013a) Maintenance agonist treatments for opiate-dependent pregnant women. Cochrane Database Syst Rev CD006318-

Minozzi S, Amato L, Vecchi S, Davoli M (2013b) Maintenance agonist treatments for opiate dependent pregnant women. Cochrane Database Syst Rev CD006318-

Moretti M E (2009) Psychotropic drugs in lactation--Motherisk Update 2008. Can J Clin Pharmacol 16: e49-e57

Morgan J F, Lacey J H, Chung E (2006) Risk of postnatal depression, miscarriage, and preterm birth in bulimia nervosa: retrospective controlled study. Psychosom Med 68: $487-492$ 
Mortensen J T, Olsen J, Larsen H, Bendsen J, Obel C, Sorensen H T (2003) Psychomotor development in children exposed in utero to benzodiazepines, antidepressants, neuroleptics, and anti-epileptics. Eur J Epidemiol 18: 769-771

Moses-Kolko E L, Bogen D, Perel J, Bregar A, Uhl K, Levin B, Wisner K L (2005) Neonatal signs after late in utero exposure to serotonin reuptake inhibitors: literature review and implications for clinical applications. JAMA 293: 2372-2383

Munk-Olsen T, Laursen T M, Mendelson T, Pedersen C B, Mors O, Mortensen P B (2009) Risks and predictors of readmission for a mental disorder during the postpartum period. Arch Gen Psychiatry 66: 189-195

Munk-Olsen T, Laursen T M, Pedersen C B, Mors O, Mortensen P B (2006) New parents and mental disorders: a population-based register study. JAMA 296: 25822589

Murray L, Arteche A, Fearon P, Halligan S, Croudace T, Cooper P (2010) The effects of maternal postnatal depression and child sex on academic performance at age 16 years: a developmental approach. J Child Psychol Psychiatry 51: 1150-1159

Nanovskaya T N, Oncken C, Fokina V M, Feinn R S, Clark S M, West H, Jain S K, Ahmed M S, Hankins G D (2016) Bupropion sustained release for pregnant smokers: a randomized, placebo-controlled trial. Am J Obstet Gynecol

Nast I, Bolten M, Meinlschmidt G, Hellhammer D H (2013) How to measure prenatal stress? A systematic review of psychometric instruments to assess psychosocial stress during pregnancy. Paediatr Perinat Epidemiol 27: 313-322

National Institute for Health and Care Excellence (2004) Eating disorders in over 8s: management. NICE Guidelines CG9:

National Institute for Health and Care Excellence (2007) Methadone and buprenorphine for the management of opioid dependence. NICE Technology Appraisal Guidance TA114:

National Institute for Health and Care Excellence (2008) Attention deficit hyperactivity disorder: diagnosis and management. NICE Guidelines CG72:

National Institute for Health and Care Excellence (2009) Depression: the treatment and management of depression in adults (update). NICE Clinical Guideline 91:

National Institute for Health and Care Excellence (2010a) Pregnancy and complex social factors: a model for service provision for pregnant women with complex social factors. NICE Guidelines CG110:

National Institute for Health and Care Excellence (2010b) Smoking: stopping in pregnancy and after childbirth. NICE Guidelines PH26:

National Institute for Health and Care Excellence (2010c) Weight management before, during and after pregnancy. NICE Guidelines PH27: 
National Institute for Health and Care Excellence (2012) Epilepsies: diagnosis and management. NICE Guidelines CG137:

National Institute for Health and Care Excellence (2013) Fertility problems: assessment and treatment. NICE Guidelines CG156:

National Institute for Health and Care Excellence (2014) Bipolar disorder: Assessment and management. NICE Guidelines CG185:

National Institute for Health and Care Excellence (2015a) Antenatal and postnatal mental health: clinical management and service guidance. NICE Guidelines CG192:

National Institute for Health and Care Excellence (2015b) Diabetes in pregnancy: management from preconception to the postnatal period. NICE Guidelines NG3:

National Institute for Health and Care Excellence (2015c) Violence and aggression: short-term management in mental health, health and community settings. NICE Guidelines NG10:

Newham J J, Thomas S H, MacRitchie K, McElhatton P R, McAllister-Williams R H (2008) Birth weight of infants after maternal exposure to typical and atypical antipsychotics: prospective comparison study. Br J Psychiatry 192: 333-337

Newport D J, Calamaras M R, DeVane C L, Donovan J, Beach A J, Winn S, Knight B T, Gibson B B, Viguera A C, Owens M J, Nemeroff C B, Stowe Z N (2007a) Atypical antipsychotic administration during late pregnancy: placental passage and obstetrical outcomes. Am J Psychiatry 164: 1214-1220

Newport D J, Calamaras M R, DeVane C L, Donovan J, Beach A J, Winn S, Knight B T, Gibson B B, Viguera A C, Owens M J, Nemeroff C B, Stowe Z N (2007b) Atypical antipsychotic administration during late pregnancy: placental passage and obstetrical outcomes. Am J Psychiatry 164: 1214-1220

Newport D J, Pennell P B, Calamaras M R, Ritchie J C, Newman M, Knight B, Viguera A C, Liporace J, Stowe Z N (2008) Lamotrigine in breast milk and nursing infants: determination of exposure. Pediatrics 122: e223-e231

Newport D J, Viguera A C, Beach A J, Ritchie J C, Cohen L S, Stowe Z N (2005) Lithium placental passage and obstetrical outcome: implications for clinical management during late pregnancy. Am J Psychiatry 162: 2162-2170

O'Connor T G, Heron J, Golding J, Glover V (2003) Maternal antenatal anxiety and behavioural/emotional problems in children: a test of a programming hypothesis. $\mathrm{J}$ Child Psychol Psychiatry 44: 1025-1036

O'Donnell K J, Glover V, Barker E D, O'Connor T G (2014) The persisting effect of maternal mood in pregnancy on childhood psychopathology. Dev Psychopathol 26: $393-403$

O'Leary C M, Nassar N, Kurinczuk J J, Bower C (2009) The effect of maternal alcohol consumption on fetal growth and preterm birth. BJOG 116: 390-400 
O'Reardon J P, Cristancho M A, von Andreae C V, Cristancho P, Weiss D (2011) Acute and maintenance electroconvulsive therapy for treatment of severe major depression during the second and third trimesters of pregnancy with infant follow-up to 18 months: case report and review of the literature. J ECT 27: e23-e26

Oberlander T F, Warburton W, Misri S, Aghajanian J, Hertzman C (2006) Neonatal outcomes after prenatal exposure to selective serotonin reuptake inhibitor antidepressants and maternal depression using population-based linked health data. Arch Gen Psychiatry 63: 898-906

Olde E, van der Hart O, Kleber R, van S M (2006) Posttraumatic stress following childbirth: a review. Clin Psychol Rev 26: 1-16

Orsolini L, Bellantuono C (2015) Serotonin reuptake inhibitors and breastfeeding: a systematic review. Hum Psychopharmacol 30: 4-20

Paluzzi A, Bain P G, Liu X, Yianni J, Kumarendran K, Aziz T Z (2006) Pregnancy in dystonic women with in situ deep brain stimulators. Mov Disord 21: 695-698

Parikh T, Goyal D, Scarff J R, Lippmann S (2014) Antipsychotic drugs and safety concerns for breast-feeding infants. South Med J 107: 686-688

Pawlby S, Hay D, Sharp D, Waters C S, Pariante C M (2011) Antenatal depression and offspring psychopathology: the influence of childhood maltreatment. Br J Psychiatry 199: 106-112

Pawlby S, Hay D F, Sharp D, Waters C S, O'Keane V (2009) Antenatal depression predicts depression in adolescent offspring: prospective longitudinal communitybased study. J Affect Disord 113: 236-243

Pedersen L H, Henriksen T B, Olsen J (2010) Fetal exposure to antidepressants and normal milestone development at 6 and 19 months of age. Pediatrics 125: e600-e608

Peng M, Gao K, Ding Y, Ou J, Calabrese J R, Wu R, Zhao J (2013) Effects of prenatal exposure to atypical antipsychotics on postnatal development and growth of infants: a case-controlled, prospective study. Psychopharmacology (Berl) 228: 577584

Pennell P B, Klein A M, Browning N, Baker G A, Clayton-Smith J, Kalayjian L A, Liporace J D, Privitera M, Crawford T, Loring D W, Meador K J (2012) Differential effects of antiepileptic drugs on neonatal outcomes. Epilepsy Behav 24: 449-456

Petersen I, McCrea R L, Sammon C J, Osborn D P, Evans S J, Cowen P J, Freemantle N, Nazareth I (2016) Risks and benefits of psychotropic medication in pregnancy: cohort studies based on UK electronic primary care health records. Health Technol Assess 20: 1-176

Plant D T, Barker E D, Waters C S, Pawlby S, Pariante C M (2013) Intergenerational transmission of maltreatment and psychopathology: the role of antenatal depression. Psychol Med 43: 519-528 
Plant D T, Pariante C M, Sharp D, Pawlby S (2015a) Maternal depression during pregnancy and offspring depression in adulthood: role of child maltreatment. Br J Psychiatry 207: 213-220

Plant D T, Pariante C M, Sharp D, Pawlby S (2015b) Maternal depression during pregnancy and offspring depression in adulthood: role of child maltreatment. $\mathrm{Br} \mathbf{J}$ Psychiatry 207: 213-220

Pottegard A, Hallas J, Andersen J T, Lokkegaard E C, Dideriksen D, Aagaard L, Damkier P (2014) First-trimester exposure to methylphenidate: a population-based cohort study. J Clin Psychiatry 75: e88-e93

Ramakrishnan A, Lee L J, Mitchell L E, Agopian A J (2015) Maternal Hypertension During Pregnancy and the Risk of Congenital Heart Defects in Offspring: A Systematic Review and Meta-analysis. Pediatr Cardiol 36: 1442-1451

Raoof N T, Pearson R M, Turner P (1989) Lithium inhibits human sperm motility in vitro. Br J Clin Pharmacol 28: 715-717

Rasmussen K M, Catalano P M, Yaktine A L (2009) New guidelines for weight gain during pregnancy: what obstetrician/gynecologists should know. Curr Opin Obstet Gynecol 21: 521-526

Reed P, Sermin N, Appleby L, Faragher B (1999) A comparison of clinical response to electroconvulsive therapy in puerperal and non-puerperal psychoses. J Affect Disord 54: 255-260

Reimers A (2014) New antiepileptic drugs and women. Seizure 23: 585-591

Reis M, Kallen B (2008) Maternal use of antipsychotics in early pregnancy and delivery outcome. J Clin Psychopharmacol 28: 279-288

Reis M, Kallen B (2010) Delivery outcome after maternal use of antidepressant drugs in pregnancy: an update using Swedish data. Psychol Med 40: 1723-1733

Reynolds-May M F, Kenna H A, Marsh W, Stemmle P G, Wang P, Ketter T A, Rasgon N L (2014) Evaluation of reproductive function in women treated for bipolar disorder compared to healthy controls. Bipolar Disord 16: 37-47

Richardson J L, Stephens S, Yates L M, Diav-Citrin O, Arnon J, Beghin D, Kayser A, Kennedy D, Cupitt D, Te W B, Peltonen M, Kaplan Y C, Thomas S H (2016) Pregnancy outcomes after maternal varenicline use; analysis of surveillance data collected by the European Network of Teratology Information Services. Reprod Toxicol 67: 26-34

Robertson E, Grace S, Wallington T, Stewart D E (2004) Antenatal risk factors for postpartum depression: a synthesis of recent literature. Gen Hosp Psychiatry 26: 289295

Ross L E, Grigoriadis S, Mamisashvili L, VonderPorten E H, Roerecke M, Rehm J, Dennis C L, Koren G, Steiner M, Mousmanis P, Cheung A (2013) Selected pregnancy 
and delivery outcomes after exposure to antidepressant medication: a systematic review and meta-analysis. JAMA Psychiatry 70: 436-443

Royal College of Physicians (2010) Passive smoking and children. A report by the Tobacco Advisory Group of the Royal College of Physicians.

Royal College of Psychiatrists (2012) The ECT handbook. 3rd:

Ryan L, Ehrlich S, Finnegan L (1987) Cocaine abuse in pregnancy: effects on the fetus and newborn. Neurotoxicol Teratol 9: 295-299

Sadowski A, Todorow M, Yazdani B P, Koren G, Nulman I (2013) Pregnancy outcomes following maternal exposure to second-generation antipsychotics given with other psychotropic drugs: a cohort study. BMJ Open 3:

Salisbury A L, O'Grady K E, Battle C L, Wisner K L, Anderson G M, Stroud L R, Miller-Loncar C L, Young M E, Lester B M (2016) The Roles of Maternal Depression, Serotonin Reuptake Inhibitor Treatment, and Concomitant Benzodiazepine Use on Infant Neurobehavioral Functioning Over the First Postnatal Month. Am J Psychiatry 173: 147-157

Sandman C A, Davis E P, Buss C, Glynn L M (2011) Prenatal programming of human neurological function. Int J Pept 2011: 837596-

Schou M (1976) What happened later to the lithium babies? A follow-up study of children born without malformations. Acta Psychiatr Scand 54: 193-197

Scottish Intercollegiate Guidelines Network (2012) Management of perinatal mood disorders. SIGN National Clinical Guidelines 127:

Shah N, Howard L (2006) Screening for smoking and substance misuse in pregnant women with mental illness. Psychiat Bull 30: 294-297

Shen Z Q, Gao S Y, Li S X, Zhang T N, Liu C X, Lv H C, Zhang Y, Gong T T, Xu X, Ji C, Wu Q J, Li D (2016) Sertraline use in the first trimester and risk of congenital anomalies: a systemic review and meta-analysis of cohort studies. Br J Clin Pharmacol

Skagerstrom J, Chang G, Nilsen P (2011) Predictors of drinking during pregnancy: a systematic review. J Womens Health (Larchmt ) 20: 901-913

Slone D, Siskind V, Heinonen O P, Monson R R, Kaufman D W, Shapiro S (1977) Antenatal exposure to the phenothiazines in relation to congenital malformations, perinatal mortality rate, birth weight, and intelligence quotient score. Am J Obstet Gynecol 128: 486-488

Smink F R, van H D, Hoek H W (2012) Epidemiology of eating disorders: incidence, prevalence and mortality rates. Curr Psychiatry Rep 14: 406-414 
Smith L, Yonekura M L, Wallace T, Berman N, Kuo J, Berkowitz C (2003) Effects of prenatal methamphetamine exposure on fetal growth and drug withdrawal symptoms in infants born at term. J Dev Behav Pediatr 24: 17-23

Solmi F, Sallis H, Stahl D, Treasure J, Micali N (2014) Low birth weight in the offspring of women with anorexia nervosa. Epidemiol Rev 36: 49-56

Soussan C, Gouraud A, Portolan G, Jean-Pastor M J, Pecriaux C, Montastruc J L, Damase-Michel C, Lacroix I (2014) Drug-induced adverse reactions via breastfeeding: a descriptive study in the French Pharmacovigilance Database. Eur J Clin Pharmacol 70: 1361-1366

Speisman B B, Storch E A, Abramowitz J S (2011) Postpartum obsessive-compulsive disorder. J Obstet Gynecol Neonatal Nurs 40: 680-690

Steer R A, Scholl T O, Hediger M L, Fischer R L (1992) Self-reported depression and negative pregnancy outcomes. J Clin Epidemiol 45: 1093-1099

Suri R, Altshuler L, Hendrick V, Rasgon N, Lee E, Mintz J (2004) The impact of depression and fluoxetine treatment on obstetrical outcome. Arch Womens Ment Health 7: 193-200

Sutton C, Murray L, Glover V (2012) Support from the start: Effective programmes from birth to two years. Journal of Children's Services 7: 18-29

Svalheim S, Sveberg L, Mochol M, Tauboll E (2015) Interactions between antiepileptic drugs and hormones. Seizure 28: 12-17

Taborelli E, Easter A, Keefe R, Schmidt U, Treasure J, Micali N (2015) Transition to motherhood in women with eating disorders: A qualitative study. Psychol Psychother

Talge N M, Neal C, Glover V (2007) Antenatal maternal stress and long-term effects on child neurodevelopment: how and why? J Child Psychol Psychiatry 48: 245-261

Terplan M, Ramanadhan S, Locke A, Longinaker N, Lui S (2015a) Psychosocial interventions for pregnant women in outpatient illicit drug treatment programs compared to other interventions. Cochrane Database Syst Rev CD006037-

Terplan M, Ramanadhan S, Locke A, Longinaker N, Lui S (2015b) Psychosocial interventions for pregnant women in outpatient illicit drug treatment programs compared to other interventions. Cochrane Database Syst Rev CD006037-

Terrana N, Koren G, Pivovarov J, Etwel F, Nulman I (2015) Pregnancy Outcomes Following In Utero Exposure to Second-Generation Antipsychotics: A Systematic Review and Meta-Analysis. J Clin Psychopharmacol 35: 559-565

Thyagarajan V, Robin C C, Wurst K E, Ephross S A, Seeger J D (2012) Bupropion therapy in pregnancy and the occurrence of cardiovascular malformations in infants. Pharmacoepidemiol Drug Saf 21: 1240-1242 
Tierney S, Fox J R, Butterfield C, Stringer E, Furber C (2011) Treading the tightrope between motherhood and an eating disorder: a qualitative study. Int J Nurs Stud 48: 1223-1233

Toh S, Li Q, Cheetham T C, Cooper W O, Davis R L, Dublin S, Hammad T A, Li D K, Pawloski P A, Pinheiro S P, Raebel M A, Scott P E, Smith D H, Bobo W V, Lawrence J M, Dashevsky I, Haffenreffer K, Avalos L A, Andrade S E (2013) Prevalence and trends in the use of antipsychotic medications during pregnancy in the U.S., 2001-2007: a population-based study of 585,615 deliveries. Arch Womens Ment Health

Tomson T, Battino D (2012) Teratogenic effects of antiepileptic drugs. Lancet Neurol 11: $803-813$

Tomson T, Landmark C J, Battino D (2013) Antiepileptic drug treatment in pregnancy: changes in drug disposition and their clinical implications. Epilepsia 54: $405-414$

Uguz F (2016) Second-Generation Antipsychotics During the Lactation Period: A Comparative Systematic Review on Infant Safety. J Clin Psychopharmacol 36: 244252

Vajda F J, O'Brien T J, Graham J, Lander C M, Eadie M J (2016) Is carbamazepine a human teratogen? J Clin Neurosci 23: 34-37

Veiby G, Daltveit A K, Engelsen B A, Gilhus N E (2014) Fetal growth restriction and birth defects with newer and older antiepileptic drugs during pregnancy. J Neurol 261: 579-588

Viale L, Allotey J, Cheong-See F, Arroyo-Manzano D, McCorry D, Bagary M, Mignini L, Khan K S, Zamora J, Thangaratinam S (2015) Epilepsy in pregnancy and reproductive outcomes: a systematic review and meta-analysis. Lancet 386: 18451852

Viggedal G, Hagberg B S, Laegreid L, Aronsson M (1993) Mental development in late infancy after prenatal exposure to benzodiazepines--a prospective study. J Child Psychol Psychiatry 34: 295-305

Vigod S N, Gomes T, Wilton A S, Taylor V H, Ray J G (2015) Antipsychotic drug use in pregnancy: high dimensional, propensity matched, population based cohort study. Brit Med J 350: h2298-

Vigod S N, Kurdyak P A, Dennis C L, Gruneir A, Newman A, Seeman M V, Rochon P A, Anderson G M, Grigoriadis S, Ray J G (2014) Maternal and newborn outcomes among women with schizophrenia: a retrospective population-based cohort study. BJOG 121: 566-574

Viguera A C, Newport D J, Ritchie J, Stowe Z, Whitfield T, Mogielnicki J, Baldessarini R J, Zurick A, Cohen L S (2007a) Lithium in breast milk and nursing infants: clinical implications. Am J Psychiatry 164: 342-345 
Viguera A C, Whitfield T, Baldessarini R J, Newport D J, Stowe Z, Reminick A, Zurick A, Cohen L S (2007b) Risk of recurrence in women with bipolar disorder during pregnancy: prospective study of mood stabilizer discontinuation. Am J Psychiatry 164: 1817-1824

Wang L H, Lin H C, Lin C C, Chen Y H, Lin H C (2010) Increased risk of adverse pregnancy outcomes in women receiving zolpidem during pregnancy. Clin Pharmacol Ther 88: 369-374

Warburton W, Hertzman C, Oberlander T F (2010) A register study of the impact of stopping third trimester selective serotonin reuptake inhibitor exposure on neonatal health. Acta Psychiatr Scand 121: 471-479

Webb R T, Wicks S, Dalman C, Pickles A R, Appleby L, Mortensen P B, Haglund B, Abel K M (2010) Influence of environmental factors in higher risk of sudden infant death syndrome linked with parental mental illness. Arch Gen Psychiatry 67: 69-77

Weinstein M R (1980) Lithium treatment of women during pregnancy and the postdelivery period. $421-429$

Wesseloo R, Kamperman A M, Munk-Olsen T, Pop V J, Kushner S A, Bergink V (2016) Risk of Postpartum Relapse in Bipolar Disorder and Postpartum Psychosis: A Systematic Review and Meta-Analysis. Am J Psychiatry 173: 117-127

Whitworth M, Dowswell T (2009) Routine pre-pregnancy health promotion for improving pregnancy outcomes. Cochrane Database Syst Rev CD007536-

Wikner B N, Kallen B (2011) Are hypnotic benzodiazepine receptor agonists teratogenic in humans? J Clin Psychopharmacol 31: 356-359

Wikner B N, Stiller C O, Bergman U, Asker C, Kallen B (2007) Use of benzodiazepines and benzodiazepine receptor agonists during pregnancy: neonatal outcome and congenital malformations. Pharmacoepidemiol Drug Saf 16: 1203-1210

Wilens T E (2003) Does the medicating ADHD increase or decrease the risk for later substance abuse? Rev Bras Psiquiatr 25: 127-128

Winterfeld U, Merlob P, Baud D, Rousson V, Panchaud A, Rothuizen L E, Bernard N, Vial T, Yates L M, Pistelli A, Ellfolk M, Eleftheriou G, de Vries L C, JonvilleBera A P, Kadioglu M, Biollaz J, Buclin T (2016) Pregnancy outcome following maternal exposure to pregabalin may call for concern. Neurology 86: 2251-2257

Wisner K L, Sit D K, Hanusa B H, Moses-Kolko E L, Bogen D L, Hunker D F, Perel J M, Jones-Ivy S, Bodnar L M, Singer L T (2009) Major depression and antidepressant treatment: impact on pregnancy and neonatal outcomes. Am J Psychiatry 166: 557-566

Wlodarczyk B J, Palacios A M, George T M, Finnell R H (2012) Antiepileptic drugs and pregnancy outcomes. Am J Med Genet A 158A: 2071-2090 
World Health Organisation (2014) Guidelines for the identification and management of substance use and substance use disorders in pregnancy.

Wurst K E, Zedler B K, Joyce A R, Sasinowski M, Murrelle E L (2016) A Swedish Population-based Study of Adverse Birth Outcomes among Pregnant Women Treated with Buprenorphine or Methadone: Preliminary Findings. Subst Abuse 10: 89-97

Yakoob M Y, Bateman B T, Ho E, Hernandez-Diaz S, Franklin J M, Goodman J E, Hoban R A (2013) The risk of congenital malformations associated with exposure to beta-blockers early in pregnancy: a meta-analysis. Hypertension 62: 375-381

Yonkers K A, Gotman N, Smith M V, Forray A, Belanger K, Brunetto W L, Lin H, Burkman R T, Zelop C M, Lockwood C J (2011) Does antidepressant use attenuate the risk of a major depressive episode in pregnancy? Epidemiology 22: 848-854

Zedler B K, Mann A L, Kim M M, Amick H R, Joyce A R, Murrelle E L, Jones H E (2016) Buprenorphine compared with methadone to treat pregnant women with opioid use disorder: a systematic review and meta-analysis of safety in the mother, fetus and child. Addiction

Zhu S H, Valbo A (2002) Depression and smoking during pregnancy. Addict Behav 27: 649-658

Zuckerman B, Amaro H, Bauchner H, Cabral H (1989) Depressive symptoms during pregnancy: relationship to poor health behaviors. Am J Obstet Gynecol 160: 11071111 